Universidad de Lima

Facultad de Ingeniería Industrial

Carrera de Ingeniería Industrial

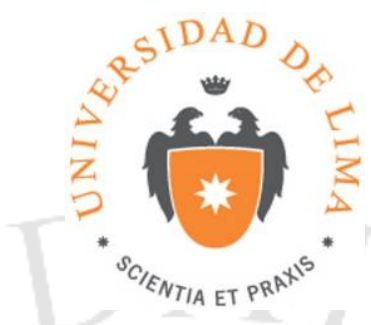

\title{
ANÁLISIS COMPARATIVO DE LOS PARADIGMAS DE SIMULACIÓN
}

Trabajo de investigación para optar el título profesional de Ingeniero Industrial

\section{Alfonso Tullio Sarmiento Vásquez \\ Código 19880671}

Asesor

Julio Padilla Solís

Lima - Perú

Mayo de 2016 


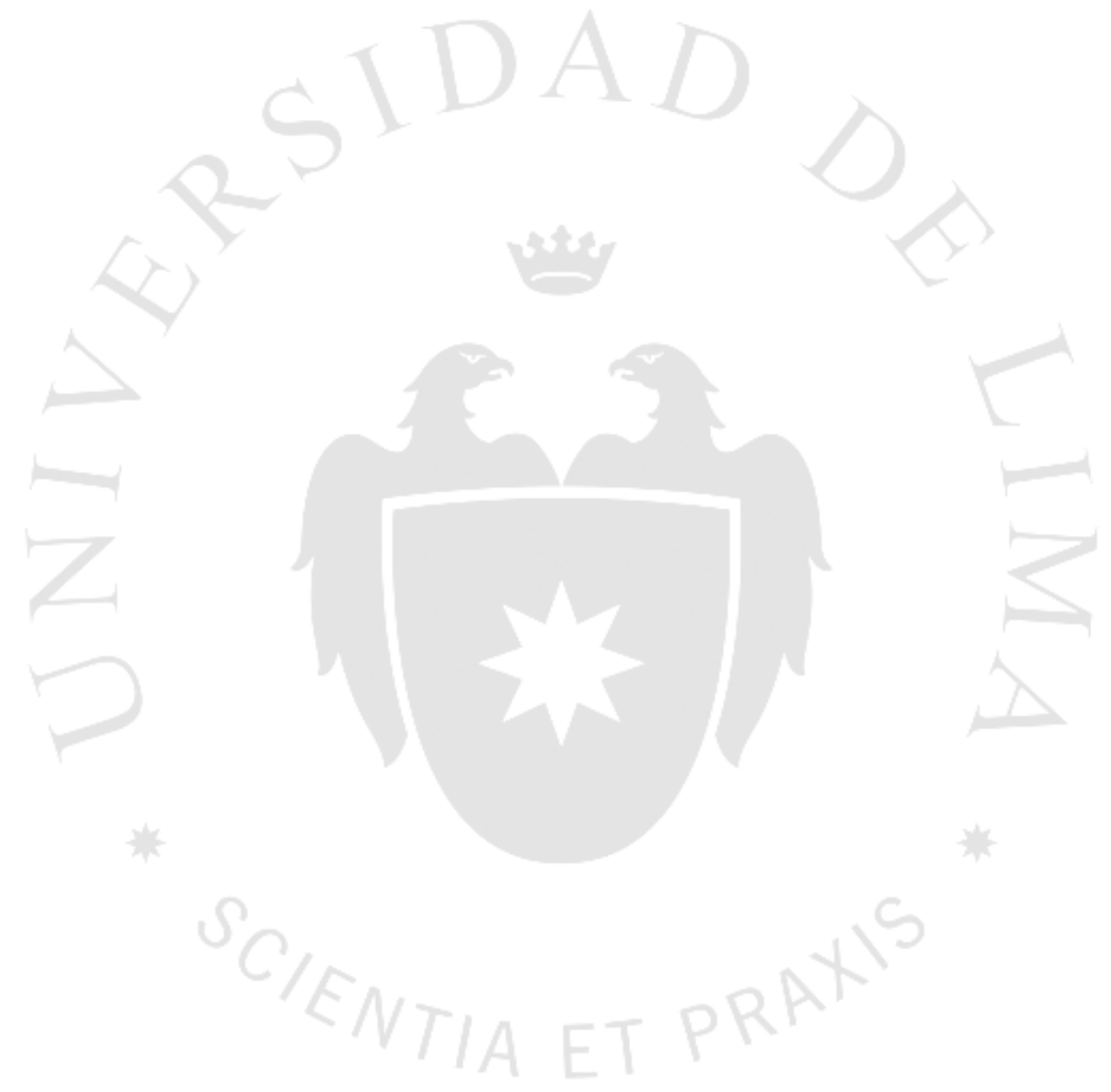




\section{ANÁLISIS COMPARATIVO DE LOS PARADIGMAS DE SIMULACIÓN}




\section{TABLA DE CONTENIDO}

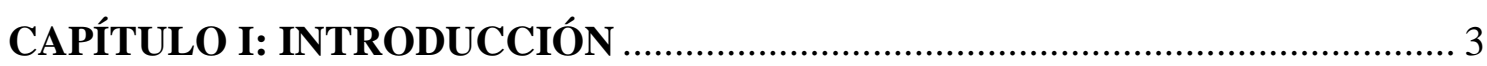

1.1. Objetivos del trabajo de investigación....................................................5

\section{CAPÍTULO II: ANÁLISIS CUALITATIVO DE LOS PARADIGMAS DE}

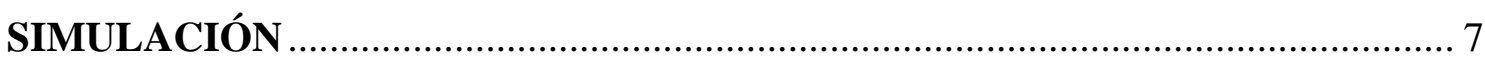

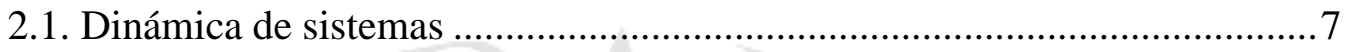

2.1.1. Fase de modelamiento cualitativo ................................................... 7

2.1.2. Fase de modelamiento cuantitativo ............................................... 10

2.2. Simulación de eventos discretos ........................................................... 13

2.3. Simulación basada en agentes ........................................................... 20

2.4. Ejemplo comparativo de los paradigmas aplicado a la simulación de tráfico

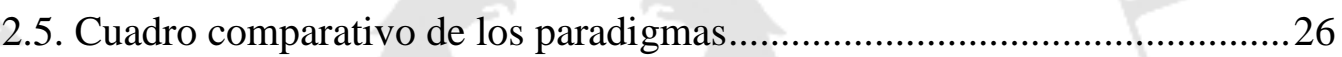

CAPÍTULO III: CASO DE ESTUDIO: E-BIKE SAN REMO .............................. 29

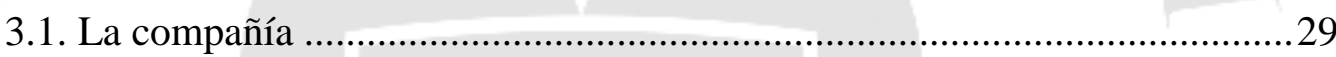

3.2. El producto y el proceso de fabricación .................................................22

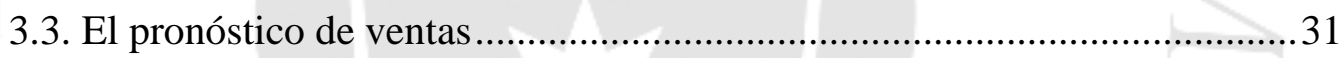

3.3.1. Modelo de difusión tecnológica............................................................. 31

3.3.2. Segmento de mercado y parámetros del modelo ....................................35

3.4. Modelo de simulación de eventos discretos ...............................................36

3.4.1. Valores promedio de las variables ................................................. 40

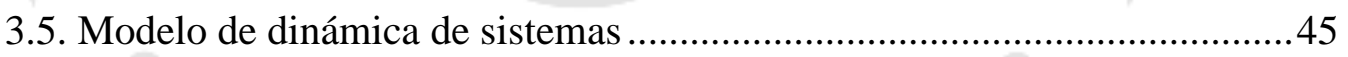

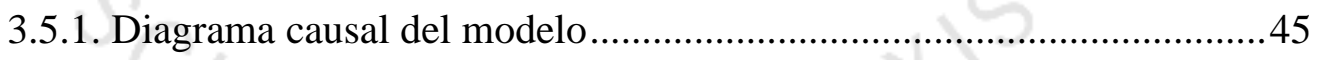

3.5.2. Diagrama de Forrester del modelo ....................................................50

3.6. Modelo de simulación basada en agentes ................................................53

3.7. Comparación cuantitativa de los paradigmas .............................................60

3.7.1. Escenario 1: Entendiendo los procesos..............................................61

3.7.2. Escenario 2: Entendiendo el mercado..................................................63

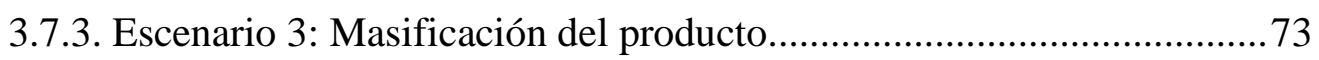

3.7.4. Escenario 4: Trabajando dos modelos de bicicletas ............................ 77

3.7.5. Escenario 5: Decisiones heterogéneas de compra .............................. 82

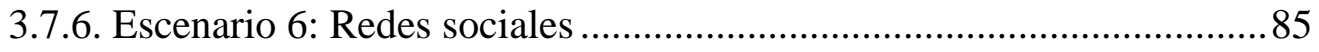


3.7.7. Resumen comparativo de los escenarios .88

CONCLUSIONES 94

RECOMENDACIONES PARA TRABAJO FUTURO 97 


\section{ÍNDICE DE TABLAS}

Tabla 2.1. Características de los paradigmas de simulación........................................26

Tabla 3.1. Valores promedio de los tiempos por replicación. ........................................ 41

Tabla 3.2. Valores promedio de las variables por replicación....................................... 41

Tabla 3.3. Probabilidades de compra por efecto de la publicidad. ................................56

Tabla 3.4. Desempeño de los paradigmas por escenario analizado............................... 88 


\section{ÍNDICE DE FIGURAS}

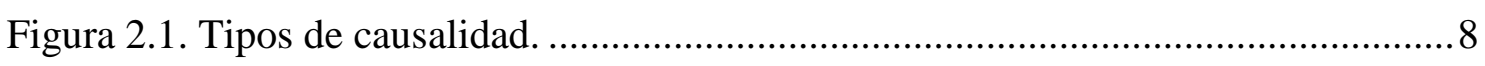

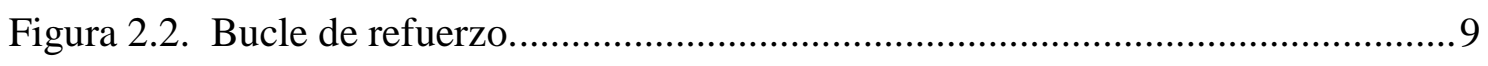

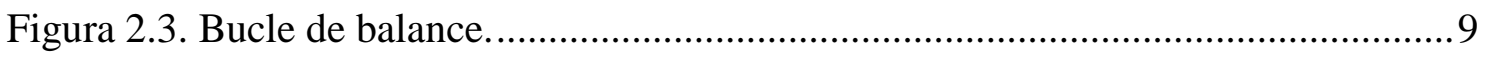

Figura 2.4. Variables del diagrama de Forrester...................................................... 11

Figura 2.5. Gráfico por eventos para un sistema de colas. ........................................... 16

Figura 2.6. Diagrama Cíclico de Actividades para un sistema de colas......................... 17

Figura 2.7. Modelo por procesos para un sistema de colas. ......................................... 17

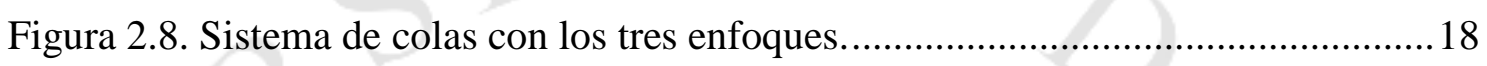

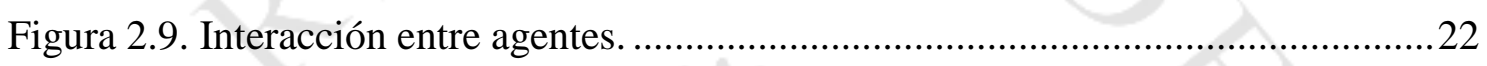

Figura 2.10. Paradigmas de simulación en modelamiento de tráfico. ...........................24

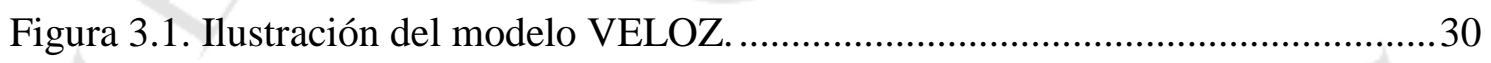

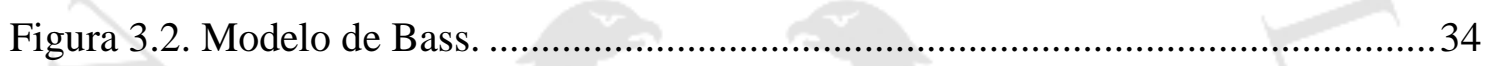

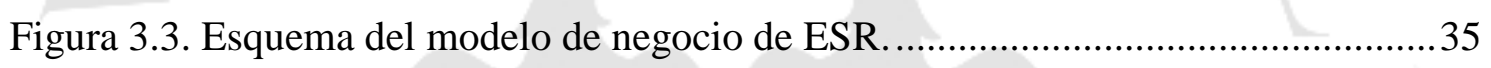

Figura 3.4. Modelo de negocio de ESR en ARENA. .................................................. 37

Figura 3.5. Resultados de tres replicaciones de Compradores y Mercado Potencial. .... 38

Figura 3.6. Resultados de tres replicaciones de la Tasa de Adopción............................39

Figura 3.7. Resultados de tres replicaciones de las Ordenes de Producción. .................. 40

Figura 3.8. Valores promedio de Compradores y Mercado Potencial........................... 43

Figura 3.9. Valor promedio de Tasa de Adopción y Ordenes de Producción. ................ 44

Figura 3.10. Valor promedio del Porcentaje de Utilización de las líneas de producción.

45

Figura 3.11. Diagrama causal para el modelo de negocio de ESR ..............................46

Figura 3.12. Diagrama causal para el bucle de balance B5 .......................................49

Figura 3.13. Diagrama causal para el bucle de balance B6....................................... 49

Figura 3.14. Diagrama de Forrester para el modelo de negocio de ESR........................50

Figura 3.15. Comparación SD y DES: Compradores y Mercado Potencial....................51

Figura 3.16. Comparación SD y DES: Tasa de Adopción.............................................52

Figura 3.17. Comparación SD y DES: Ordenes de Producción. ....................................52

Figura 3.18. Comparación SD y DES: Utilización de las Líneas...................................53

Figura 3.19. Diagrama de bloques con la lógica del modelo de ABS. ............................55

Figura 3.20. Cronograma de iteraciones en ABS. .....................................................58 
Figura 3.21. Comparación ABS y DES: Compradores y Mercado Potencial. ................59

Figura 3.22. Comparación ABS y DES: Ordenes de Producción...................................60

Figura 3.23. Modificaciones del modelo original en ARENA para el Escenario 1........61

Figura 3.24. Modificaciones del modelo original en VENSIM para el Escenario 1.....63

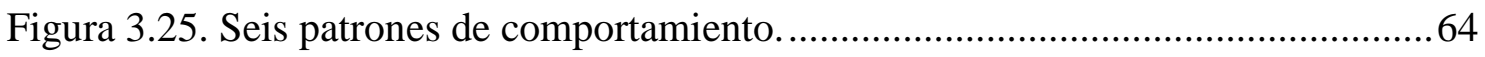

Figura 3.26. Diagrama de Forrester para el pronóstico de la demanda. ..........................66

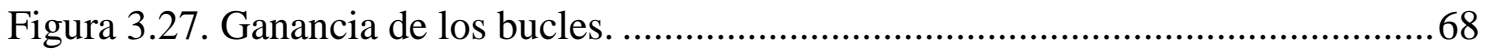

Figura 3.28. Comportamiento de la curva Compradores...............................................69

Figura 3.29. Curva de percentiles: análisis horizontal............................................. 71

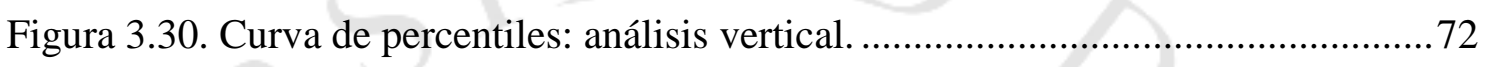

Figura 3.31. Mercado Potencial y Compradores para el Escenario 3........................... 75

Figura 3.32. Tasa de Adopción para el Escenario 3 ................................................. 76

Figura 3.33. Modificaciones del modelo original en ARENA para el Escenario 4........78

Figura 3.34. Comparación SD y DES: Ordenes de Producción regla FIFO................... 80

Figura 3.35. Comparación SD y DES: Ordenes de Producción-prioridad para

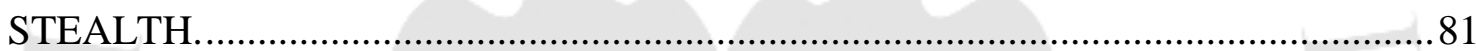

Figura 3.36. Probabilidad variable de adquisición del producto. .................................. 82

Figura 3.37. Mercado Potencial y Compradores para el Escenario 5 ........................... 83

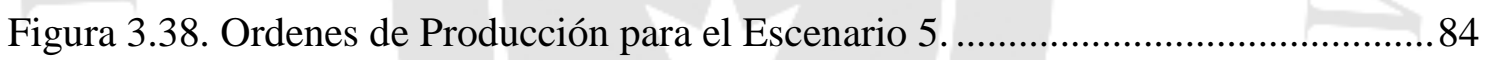

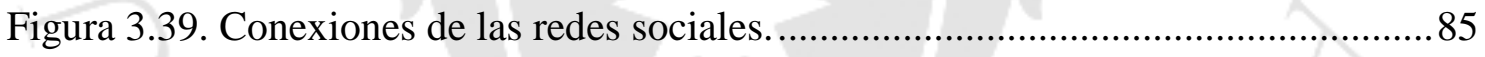

Figura 3.40. Mercado Potencial y Compradores para el Escenario 6.............................. 86

Figura 3.41. Ordenes de Producción para el Escenario 6.............................................. 87 


\section{ÍNDICE DE ANEXOS}

Anexo 1: Código en ARENA del modelo de negocio de ESR ...................................... 105

Anexo 2: Código en VENSIM del modelo de negocio de ESR ................................... 110

Anexo 3: Código en NETLOGO del modelo de negocio de ESR ................................ 113 


\section{RESUMEN}

Este trabajo de investigación hace un análisis cualitativo y cuantitativo de los paradigmas de la simulación. Los tres paradigmas estudiados en este trabajo son: dinámica de sistemas, simulación de eventos discretos y la simulación basada en agentes. En el análisis cualitativo, primero se detallan las definiciones y características de cada uno de los tres métodos para luego mostrar en un cuadro comparativo los elementos distintivos de cada uno de ellos. El análisis cuantitativo se realiza a través de un caso de estudio, donde primero se modela y se simula el problema a través del enfoque de cada método en particular. Después mediante diferentes escenarios se muestran las ventajas y desventajas de usar un paradigma sobre los otros. Esto se expone en un resumen comparativo de los escenarios. La integración de las características cualitativas y cuantitativas que distinguen a cada paradigma se presentan en las conclusiones. 


\begin{abstract}
This research work makes a qualitative and quantitative analysis of the simulation paradigms. The three paradigms studied in this work are: system dynamics, discrete event simulation and agent-based simulation. In the qualitative analysis, the definitions and characteristics of each of the three methods are detailed first and then the distinctive elements of each paradigm are showed in a comparative table. The quantitative analysis is performed through a case study, where first the problem is modeled and simulated through the focus of each particular method. After that, by using different scenarios it is showed the advantages and disadvantages of using a paradigm over the others. This is showed in a comparative summary of the scenarios. The integration of the qualitative and quantitative characteristics that distinguish each paradigm are presented in the conclusions.
\end{abstract}




\section{CAPÍTULO I: INTRODUCCIÓN}

La simulación se puede definir como la imitación de un sistema usando un modelo de computadora con el fin de evaluar y mejorar el desempeño del mismo (Harrell et al., 2004). Donde un sistema se entiende como una colección de elementos que funcionan juntos para conseguir una meta deseada (Blanchard, 1991). El estado de un sistema en un punto específico de tiempo se indica a través de las variables de estado.

Hay muchas formas de caracterizar los modelos de simulación. Una de las más comunes es dividirlos en los siguientes tipos: (a) estáticos y dinámicos, (b) determinísticos y estocásticos y (c) continuos y discretos (Law, 2014; Harrell et al., 2004).

Un modelo de simulación estático es una representación de un sistema en un punto particular de tiempo o uno que se use para representar un sistema que no está basado en el tiempo. Por ejemplo, la simulación de Montecarlo se utiliza para el análisis de riesgo en la introducción de un producto nuevo. Por otro lado, un modelo dinámico de simulación incluye el paso del tiempo. Un mecanismo de reloj se mueve hacia adelante en el tiempo y las variables de estado son actualizadas conforme el tiempo avanza. Este tipo de simulación es adecuada para analizar sistemas de manufactura y servicios ya que ellos operan a través del tiempo.

Un modelo de simulación que no contiene componentes probabilísticos o aleatorios se denomina determinístico. Por ejemplo, un sistema de ecuaciones describiendo una reacción química. En este tipo de modelos, una vez que son definidos los datos de entrada y el estado inicial, todos los estados futuros son determinados con certeza. Algunos sistemas, sin embargo, pueden ser modelados con algunos componentes aleatorios, como en el caso de los modelos de simulación estocásticos. Estos modelos tienen entradas aleatorias que generan también resultados aleatorios. Los sistemas de colas y muchos modelos de inventarios se modelan de esta forma.

En un modelo de simulación continuo, el estado del sistema es representado por variables dependientes que cambian continuamente a través del tiempo. Este tipo de modelos es construido definiendo las ecuaciones para un conjunto de variables de 
estado cuyo comportamiento dinámico simula el sistema real. Por ejemplo, el flujo de agua través de una tubería. En los modelos de simulación discretos las variables de estado cambian instantáneamente en puntos separados de tiempo. Se dice que el sistema sólo puede cambiar en un número contable de puntos en el tiempo. Por ejemplo, una línea de producción con una máquina, donde el tiempo se contabiliza al iniciar un trabajo en la máquina y al finalizar el trabajo. No se toma en consideración el paso del tiempo entre estos dos instantes ya que la variable de estado que controla la situación de la máquina no cambia durante el procesamiento del trabajo.

Un paradigma se puede definir como un conjunto de conocimientos y creencias que forman una visión del mundo y que es compartida por un grupo de personas. Es decir, es una perspectiva general de interpretar el mundo. Por lo tanto, cada paradigma de simulación es caracterizado por un conjunto de supuestos básicos y algunos conceptos subyacentes para describir el mundo. Estos supuestos, en realidad, limitan el desarrollo conceptual del modelo usado para describir el sistema de estudio. En consecuencia, la elección del paradigma apropiado es un paso importante en el proceso de desarrollo de un modelo de simulación (Behdani, 2012).

La literatura menciona tres importantes paradigmas de simulación: (1) dinámica de sistemas, (2) simulación de eventos discretos y (3) simulación basada en agentes (Borshchev, 2013; Behdani, 2012). En realidad cada uno de estos tres métodos tiene su grupo de seguidores, revistas de publicaciones y conferencias independientes que soportan sus actividades.

Algunos autores mencionan un cuarto paradigma denominado sistemas dinámicos (Serova, 2013; Yu, 2008). Este paradigma es utilizado especialmente en disciplinas de ingeniería técnica como mecánica, eléctrica y química como una parte estándar de su proceso de diseño. Sirve para diseñar y modelar el comportamiento de "sistemas físicos" a lo largo del tiempo a través de un número de variables de estado y de sistemas de ecuaciones diferenciales de varias formas sobre esas variables (Borshchev y Filippov, 2004). Las variables tienen significados físicos directos: velocidad, aceleración, presión, concentración, etc. Por ejemplo, se usa para estudiar la dinámica de los aviones o los sistemas de control en los automóviles. Debido a que este trabajo de investigación se concentrará en los tres principales paradigmas de la simulación cuyo enfoque predominante son los procesos de negocio y los sistemas sociales, no se incluirá en este análisis a los "sistemas dinámicos". 
La visión en que se centra cada uno de los tres paradigmas de simulación está asociada con las características de los modelos de simulación mencionados líneas arriba. Los tres paradigmas trabajan con modelos dinámicos de simulación. Sin embargo, la técnica de dinámica de sistemas trabaja con modelos continuos y determinísticos. La Simulación de eventos discretos es una simulación de tipo discreto enfocada mayormente en modelos estocásticos. La simulación basada en agentes es una simulación de naturaleza discreta y estocástica. Una descripción más detallada de estos tres paradigmas se hará en el capítulo 2 de este documento.

\subsection{Objetivos del trabajo de investigación}

Se han escrito muchas publicaciones sobre los tres paradigmas de simulación. La mayoría se centra en explicar cada uno de los paradigmas de forma individual. Son muy conocidos los libros de Forrester (1990), Sterman (2000) y Morecroft (2007) en el área de dinámica de sistemas. En el área de simulación de eventos discretos son tradicionales los libros de Pritsker y O'Reilly (1999) y Kelton et al. (2006). La simulación basada en agentes es la más reciente de las tres. La bibliografía asociada con ella es relativamente nueva, tal como, (Hitoshi, 2013; Railsback y Grimm, 2011).

La mayoría de las publicaciones donde se comparan los paradigmas se han hecho de a pares. Es decir, se han tomado dos de los tres paradigmas y se han analizado sus características, similitudes y diferencias. Los siguientes autores han escrito sobre la dinámica de sistemas y la simulación de eventos discretos: Brailsford, 2014b; Tacko y Robinson, 2009; Morecroft y Robinson, 2005; Sweetser, 1999. En el caso de la simulación de eventos discretos y la simulación basada en agentes tenemos a autores como: Brailsford 2014a; Heath et al., 2011; Siebers et al., 2010; Majid et al., 2009. Similares comparaciones se han hecho para el caso de la dinámica de sistemas y la simulación basada en agentes (Schieritz y Milling, 2003; Macal, 2010; Wakeland et al., 2004; Parunak et al., 1998).

Muy pocas publicaciones presentan un análisis comparativo de los tres paradigmas de forma simultánea (Marshall et al., 2015b; Serova, 2013; Behdani, 2012; Borshchev y Filippov, 2004). Más aún, éstas se centran en los aspectos netamente cualitativos. Aunque se han encontrado análisis de tipo cuantitativo, éstos se han basado en modelos extensos y a veces complejos que no han permitido extraer claramente el efecto de los paradigmas en los resultados de la situación bajo análisis. Esto se ha 
dificultado también por el hecho de no contar con los códigos de los modelos y debido a la amplia cantidad de factores y variables involucradas.

Entre los objetivos de este trabajo está el presentar una descripción breve pero detallada de los tres paradigmas y rescatar de la literatura esas comparaciones cualitativas entre ellos. Pero además mostrar con datos numéricos, mediante un caso de estudio simple, como un modelo de simulación basado en la visión de un paradigma determinado puede ser más favorable en la representación de ciertos problemas y como en ciertos casos puede ser inclusive la única opción. Para ello se utilizarán tres herramientas computacionales cada una asociada con uno de los paradigmas bajo estudio. Los códigos de los modelos se incluyen como referencia en los anexos al final del documento permitiendo que se pueda acceder a ellos para replicar los experimentos planteados. Finalmente, se busca contrastar las características cualitativas de los tres paradigmas con los resultados cuantitativos y presentar recomendaciones de trabajos futuros en esta área. 


\section{CAPÍTULO II: ANÁLISIS CUALITATIVO DE LOS PARADIGMAS DE SIMULACIÓN}

En este capítulo se hará una descripción detallada de cada uno de los tres paradigmas de simulación explicando tanto el marco conceptual que los define como el conjunto de asunciones y aspectos clave que los caracteriza en su visión del mundo. Finalmente se organizarán los elementos distintivos de los tres paradigmas en un cuadro comparativo para poder contrastarlos con los requisitos de modelamiento de futuros sistemas de estudio.

\subsection{Dinámica de sistemas}

Jay Forrester (1958) introdujo la Dinámica de Sistemas (en inglés "System Dynamics" y que denotaremos "SD") a finales de los años 50 como una metodología de modelamiento y simulación para el análisis y toma de decisiones en el largo plazo de problemas empresariales industriales. SD tiene su origen en la administración y la ingeniería de control; el método usa una perspectiva basada en la realimentación y en los retrasos de la información para entender el comportamiento dinámico de complejos sistemas físicos, biológicos y sociales (Angerhofer y Angelides, 2000). La idea esencial en $\mathrm{SD}$ es que todos los elementos en un sistema interactúan a través de relaciones causales. Estas relaciones son representadas por bucles de realimentación, los cuales controlan las interacciones entre los elementos del sistema y son la causa de su comportamiento (Rabelo et al., 2003).

El modelamiento en SD se puede dividir en dos fases: una fase cualitativa y otra fase cuantitativa, las cuales detallaremos a continuación:

\subsubsection{Fase de modelamiento cualitativo}

En esta fase se definen las relaciones de causalidad que se producen entre los elementos que integran el sistema. Esto se logra elaborando el diagrama causal, el cual es una herramienta visual para representar las estructuras de realimentación de los sistemas (Sterman, 2000). Un diagrama causal consiste de variables conectadas por flechas denotando las influencias causales entre esas variables. 
Existen dos tipos de causalidad: positiva (+) y negativa (-). El carácter de la relación se expresa asociando un signo a la flecha. En una relación positiva entre dos variables X e Y: si X se incrementa (disminuye) entonces Y se incrementa (disminuye) en una cantidad por encima (por debajo) de lo que hubiera sido si no hubiera ocurrido el cambio en X. La Figura 2.1a muestra una relación de causalidad positiva. En este caso, un mayor incremento en el presupuesto de mercadeo genera un incremento en las ventas.

Figura 2.1.

Tipos de causalidad.

a)

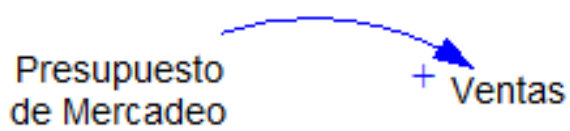

b)

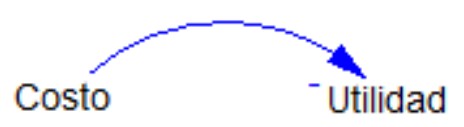

Elaboración propia.

Por otro lado, en una relación negativa entre dos variables $\mathrm{X}$ e $\mathrm{Y}$ : si $\mathrm{X}$ se incrementa (disminuye) entonces $\mathrm{Y}$ disminuye (se incrementa) en una cantidad por debajo (por encima) de lo que hubiera sido si no hubiera ocurrido el cambio en X. La Figura 2.1b muestra una relación de causalidad negativa. Se interpreta que a un mayor costo entonces la utilidad disminuye.

Cuando un grupo de variables interconectadas por relaciones causales forman un camino cerrado que comienza en una variable inicial y acaba en esa misma variable entonces se dice que ese grupo de variables forman un bucle de realimentación. Existen dos tipos de bucles: de realimentación positiva o de refuerzo y de realimentación negativa o de balance.

Un bucle de refuerzo (R) genera un comportamiento de crecimiento o decrecimiento del sistema que lo aleja del punto de equilibrio. Es decir tiende a desestabilizar los sistemas de forma exponencial. Por ejemplo, en la Figura 2.2 las variables Nacimientos y Población forman un bucle y se interpreta que: a un mayor número de nacimientos (considerando una tasa de natalidad constante) se produce un aumento en la población lo que genera a su vez que se incremente el número de nacimientos. Esto genera un crecimiento exponencial de la variable Población. 
Figura 2.2.

Bucle de refuerzo.
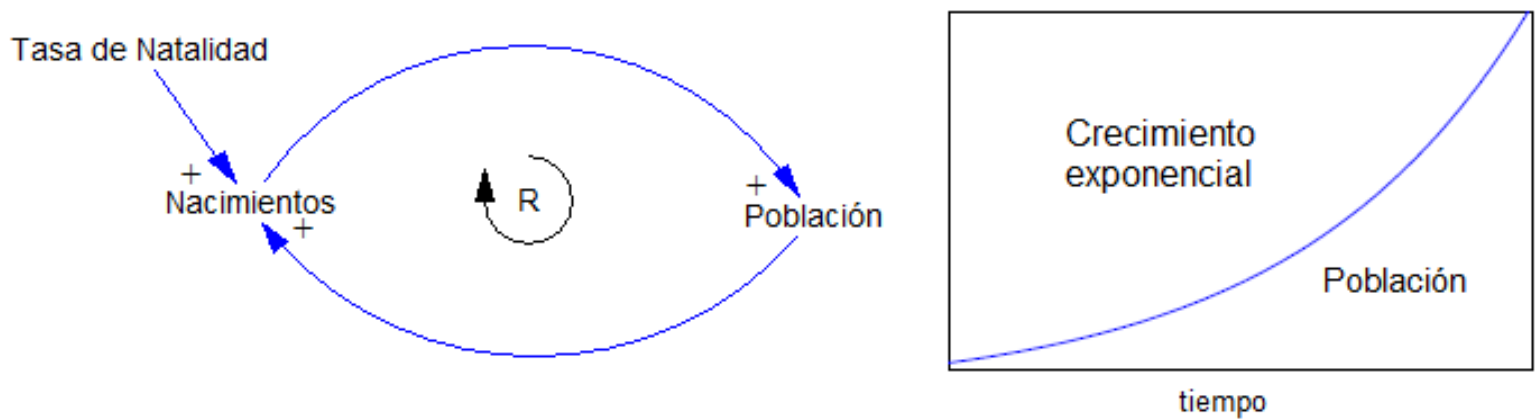

Elaboración propia.

A un bucle de balance (B) se le conoce como estabilizador o regulador y es aquel en que una variación de un elemento se transmite a lo largo del bucle de manera que genere un efecto que contrarresta la variación inicial. Por ejemplo, en la Figura 2.3 las variables Población y Muertes forman un bucle y se interpreta así: a un incremento de la población se produce un aumento en el número de muertes lo que genera a su vez (considerando una tasa de mortalidad constante) que disminuya la población. Esto genera que la variable Población tienda a buscar asintóticamente un equilibrio.

Figura 2.3.

Bucle de balance.
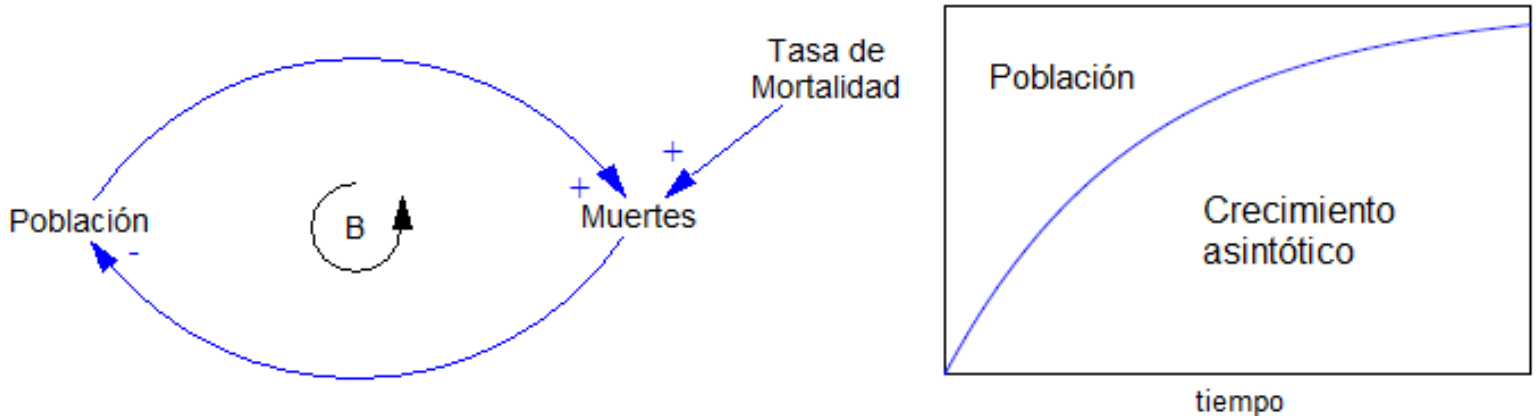

Elaboración propia.

Una regla que se suele utilizar para determinar la polaridad de un bucle de realimentación consiste en multiplicar las polaridades de las relaciones individuales que lo constituyen (Richardson, 1995). Los bucles con polaridad positiva son de refuerzo y los de polaridad negativa son de balance. En el ejemplo de la Figura 2.3, la relación "Población $\rightarrow$ Muertes" tiene una polaridad positiva y la relación "Muertes $\rightarrow$ 
Población" tiene una polaridad negativa. El producto de ambas relaciones da una polaridad negativa lo que indica balance.

La fuerza de la relación de dos variables interconectadas X e Y se puede medir mediante lo que se conoce como su Ganancia ó $\mathrm{G}(\mathrm{X} \rightarrow \mathrm{Y})$ y se define como $\mathrm{G}(\mathrm{X} \rightarrow \mathrm{Y})=\mathrm{dY} / \mathrm{dX}$. Además la Ganancia de un bucle de realimentación se define como el producto de las ganancias de las variables relacionadas (de a dos) que conforman el bucle (Kampmann, 2012).

\subsubsection{Fase de modelamiento cuantitativo}

Para poder generar un modelo cuantitativo que se pueda simular en un computador se debe traducir el diagrama causal a un diagrama de Forrester que es un paso intermedio para la obtención de las ecuaciones matemáticas que definen el comportamiento del sistema. El Diagrama de Forrester tiene dos elementos: parámetros (ó constantes) y variables. Las variables pueden ser de tres tipos (Morlán, 2010):

- Variables de nivel. Definen el estado del sistema y son las variables que acumulan magnitudes con el tiempo.

- Variables de flujo. Representan el cambio de las variables de nivel durante un periodo de tiempo. Son las derivadas de los niveles con respecto al tiempo.

- Variables auxiliares. Se utilizan para descomponer ecuaciones complejas en ecuaciones más simples que faciliten la lectura el modelo.

En la Figura 2.4 se muestra un ejemplo del Diagrama de Forrester, donde Población es una variable de nivel, los Nacimientos y las Muertes son variables de flujo y las tasas de Natalidad y Mortalidad son parámetros. Las dos nubes (a la izquierda de la variable Nacimientos y a la derecha de la variable Muertes) representan una fuente y un sumidero respectivamente. Es decir, me marcan los límites del modelo. En este caso, no me interesa analizar de donde vienen los nacimientos ni a donde van las muertes. 
Figura 2.4.

Variables del diagrama de Forrester.

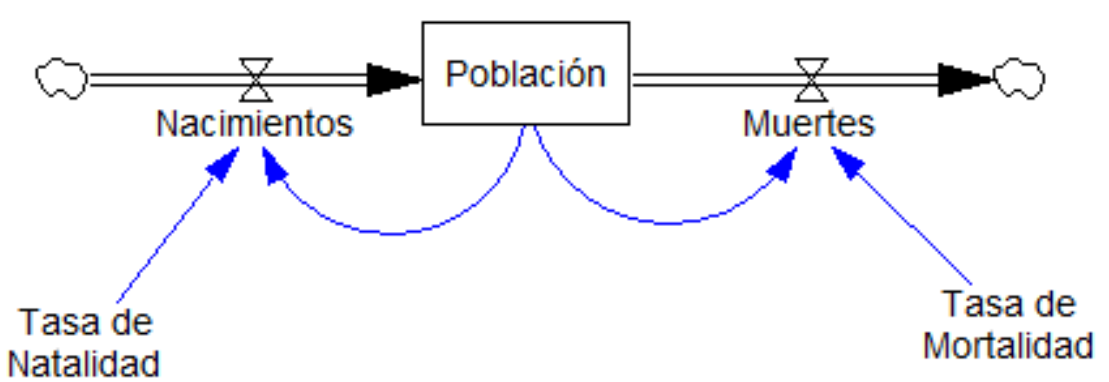

Elaboración propia.

El valor de las variables de nivel y flujo se obtiene de las siguientes ecuaciones:

- Ecuaciones de nivel. Estas ecuaciones calculan el valor de las variables de nivel como una acumulación en el tiempo de la diferencia de los flujos de entrada y salida de un proceso. Por ejemplo, el valor de la Población en el instante de tiempo t se puede expresar como la integral de la diferencia entre los Nacimientos y las Muertes, partiendo de un valor inicial de la Población en el instante t=0.

Población $(\mathrm{t})=$ Población $(0)+\int_{0}^{\mathrm{t}}[\operatorname{Nacimientos}(\mathrm{z})-\operatorname{Muertes}(\mathrm{z})] \mathrm{dz}$

- Ecuaciones de flujo. A diferencia de las variables de nivel, las ecuaciones de flujo no tienen una forma estándar. Por ejemplo, los nacimientos se pueden expresar como un porcentaje de la población dado por la tasa de natalidad.

Nacimientos $(\mathrm{t})=$ Población $(\mathrm{t}) *$ Tasa de Natalidad

Los Diagramas de Forrester representan sistemas continuos sin embargo su simulación es discreta ya que se realiza por medio de un computador. Esto significa que en lugar de manejar diferenciales de tiempo, dt, se utilizan incrementos o intervalos discretos de tiempo, $\Delta \mathrm{t}$.

A continuación se listan las principales características de SD (Dangerfield, 2014; Brailsford et. al, 2010):

- El principio fundamental en SD indica que la estructura del sistema determina su comportamiento a través del tiempo. En otras palabras, la manera en que los 
componentes separados de cualquier sistema se relacionan y afectan entre ellos determinan el comportamiento emergente del sistema como una totalidad. El componente básico usado en la construcción de un modelo es el bucle de realimentación.

- El paradigma trabaja con valores agregados (de productos, gente, etc.) y no con entidades individuales. Los objetos almacenados (como un todo) en el mismo nivel de consideran homogéneos e indistinguibles. Se dice que con el paradigma de SD se tienen una visión del bosque en vez de representar los árboles. Es decir, se representan las características de los objetos o los objetos por si mismos mediante propiedades promedio. Esto es un resultado directo de la técnica de modelamiento usada donde las variables de nivel son acumulaciones de los flujos; lo que no permite identificar los objetos simples fluyendo a través del sistema.

- Es esencialmente un método determinístico y no maneja la variabilidad de manera muy efectiva, a pesar de los esfuerzos de los vendedores de software por incluir funciones de probabilidad.

- Los modelos de SD se corren de forma muy rápida y al ser determinísticos no requieren de múltiples replicaciones.

- Representaciones lineales ${ }^{1}$ son usadas en los modelos de SD cuando se convierte una unidad de medida en otra unidad de medida (ejemplo, años en meses) o cuando la relación entre las variables es esencialmente constante en el intervalo donde se están estudiando. Sin embargo, SD permite al modelador la flexibilidad de introducir relaciones no lineales en el modelo.

- Permite el análisis de como las relaciones no lineales de causalidad entre las variables y parámetros del modelo afectan el comportamiento del sistema. Este análisis generalmente no se puede obtener mediante las técnicas de correlación usadas en otros paradigmas, ya que estas últimas sirven para detectar únicamente relaciones lineales.

Cuando una variable se incrementa en una proporción constante de otra, tal como, $y=m x+b$ 
- Es una metodología de arriba hacia abajo ${ }^{2}$ (top-down), es decir, tiene un enfoque macroscópico de ver el mundo, como una síntesis holística de un sistema de elementos los cuales están dinámicamente interconectados. Se considera que tiene un enfoque más estratégico en donde los eventos y las decisiones son vistos como formas de comportamiento y estructuras del sistema.

- Se enfoca en la dinámica del sistema por causas endógenas, es decir, los cambios en el tiempo vienen dentro de los límites internos del sistema debido a las interacciones de los componentes, aunque el estímulo inicial de esas dinámicas puede haber sido exógeno (externo a los límites del sistema).

- Pertenece a la clasificación de simulación continua, donde los flujos se asumen que cambian de forma continua con respecto al tiempo y son regidos por ecuaciones diferenciales de primer orden ${ }^{3}$.

- Aunque las tasas de los flujos son incluidas, los modelos de SD se concentran principalmente en el comportamiento de los niveles o acumuladores en el sistema. Estos son descritos por ecuaciones de integración.

- No se ignoran las variables blandas (como moral o reputación) las cuales se consideran que tienen una influencia causal en el sistema.

\subsection{Simulación de eventos discretos}

La simulación de eventos discretos (en inglés "Discrete Event Simulation" y que denotaremos "DES") fue desarrollada en los 60s en las áreas de Investigación de Operaciones e Ingeniería Industrial para ayudar a analizar y mejorar los procesos industriales y de negocios. Cabe destacar el desarrollo de GPSS (General Purpose Simulation System) por Geoffrey Gordon de IBM y que ha contribuido conceptualmente al desarrollo de los lenguajes comerciales de DES que han aparecido desde entonces.

Método que empieza en el nivel conceptual más alto y desciende hasta los detalles.

Ecuaciones donde intervienen derivadas de primer orden, es decir, derivadas de la forma dy/dx 
DES se refiere a la modelación computacional de sistemas que evolucionan en el tiempo mediante cambios instantáneos en las variables de estado. Los cambios ocurren en puntos separados del tiempo (ó intervalos discretos) accionados por eventos. Es decir, el modelo salta desde el tiempo de un evento hacia el tiempo del siguiente evento. Estas características le otorgan a DES la flexibilidad y eficiencia para ser usada en una amplia variedad de problemas.

En términos simples, DES modela el progreso de los sistemas de colas a través del tiempo. Esto lo logra representando el mundo como entidades que fluyen a través de una red de colas y actividades, en donde los recursos son compartidos entre las actividades (Robinson, 2014). En otras palabras, DES es muy útil para problemas que consisten en simulaciones de colas o redes complejas con colas, en las cuales los procesos pueden ser bien definidos y el énfasis está en representar incertidumbre a través de distribuciones estocásticas. Muchas de estas aplicaciones ocurren en las industrias de manufactura y servicio (Siebers et al., 2010).

Los conceptos principales de DES son: entidades, atributos, variables, eventos, recursos, colas y tiempo (Karnon et al., 2012; Kelton et al., 2006).

- Entidades. Son objetos dinámicos que tienen atributos, consumen recursos y entran a las colas. Las entidades cambian de estado, afectan y son afectadas por otras entidades y el estado del sistema. Usualmente son creadas, se mueven por el sistema por un lapso de tiempo y luego son eliminadas al salir del sistema. Aunque puede haber entidades que nunca dejen el sistema y se mantengan circulando de forma permanente.

- Atributo. Es una característica común de todas las entidades, pero con un valor específico que puede variar de una entidad a otra (ejemplo: edad, sexo, color, etc.). Estos valores pueden ser usados para determinar como una entidad responde a determinadas circunstancias y pueden ser modificados durante cualquier momento de la simulación.

- Variables. Representan características del sistema que son independientes de los tipos de entidades, sin embargo, éstas últimas pueden modificar las variables. 
- Evento. Es algo que sucede en un instante de tiempo y que puede cambiar a los atributos, variables o estadísticas.

- Recurso. Es un objeto que provee un servicio a una entidad. Los recursos representan personas, equipo, espacio físico, etc. La entidad captura (unidades de) un recurso cuando está disponible y las libera cuando termina de usarlo.

- Colas. Si un recurso está ocupado cuando una entidad lo requiere, entonces la entidad debe esperar, formando una cola. Las colas pueden tener capacidades máximas y reglas que definen la prioridad de las entidades en la cola como FIFO (First In Firt Out).

- Tiempo. Un reloj de simulación mantiene un registro del tiempo. A diferencia del tiempo real, el reloj de simulación no calcula todos los valores de forma continua, sino que salta del tiempo de un evento al tiempo del siguiente evento. Para ello, el reloj de simulación interactúa con el calendario de eventos (que es una lista de los eventos programados).

Los modelos de simulación discreta pueden desarrollarse a través de tres enfoques (Heath et al., 2011; Pegden, 2010; Pidd, 2004):|

- Enfoque de eventos. El sistema es modelado definiendo los cambios que ocurren al momento de los eventos. La tarea del modelador consiste en determinar los eventos que pueden cambiar el estado del sistema (el estado del sistema no cambia entre eventos) y luego desarrollar la lógica asociada con cada tipo de evento. Una simulación del sistema se produce ejecutando la lógica asociada con cada evento en una secuencia ordenada en el tiempo.

Se deben hacer las siguientes preguntas para iniciar el modelamiento de un sistema por este enfoque:

○ ¿Cómo se define el estado del sistema?

○ ¿Cuáles son los eventos que pueden cambiar el estado del sistema?

○ ¿Cuál es la lógica dentro de cada evento que define los cambios de estado?

Un modelo de un gráfico por eventos se muestra en la Figura 2.5, con un evento de arribo (con tiempo entre arribos $t_{a}$ ), una operación de servicio (con tiempo de 
servicio $t_{s}$ ) representada con un evento de inicio y un evento de fin, una cola $Q$ inicialmente vacía, y un recurso $\mathrm{S}$ con una capacidad de k unidades.

Figura 2.5.

Gráfico por eventos para un sistema de colas.

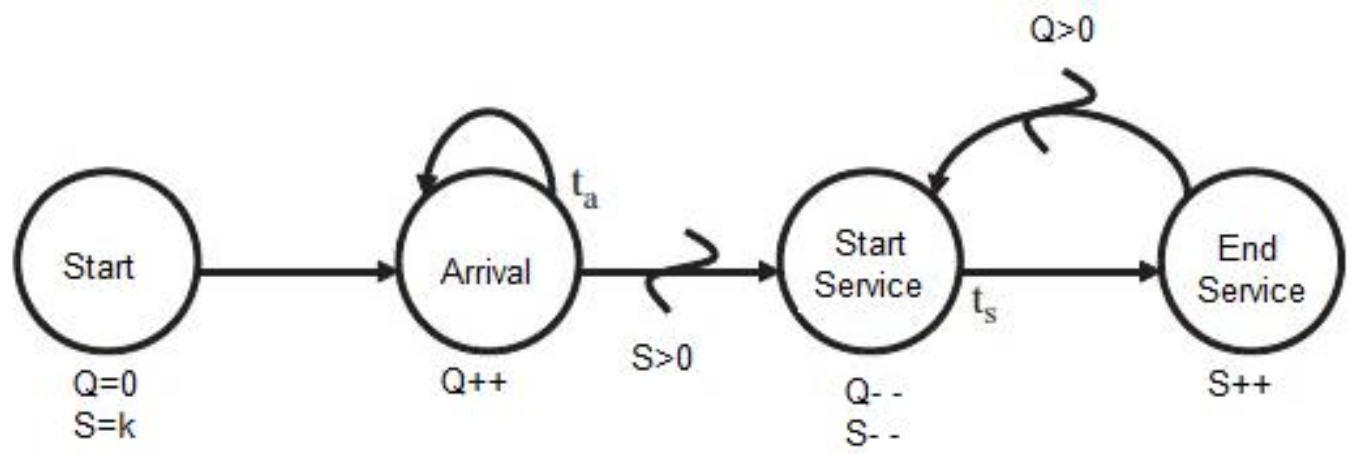

Fuente: Kesaraju, V., \& Ciarallo, F. (2012).

- Enfoque de actividades. Se originó como el método denominado escaneo de actividades El modelador describe las actividades en las que participan las entidades del sistema y determina las condiciones que causan que una actividad se inicie o finalice. Los eventos que inician o finalizan la actividad no son programados por el modelador, pero son ejecutados por las condiciones especificadas para la actividad. Mientras el tiempo de la simulación avanza, se monitorean las condiciones para iniciar o finalizar una actividad. Si las condiciones son satisfechas, son ejecutadas las acciones apropiadas para la actividad. Para asegurar que cada actividad es considerada, es necesario monitorear todo el conjunto de actividades cada vez que el tiempo avanza. Es debido a esto que este enfoque es menos eficiente que de eventos discretos.

El método de escaneo de actividades evolucionó en el denominado método de las tres fases que es un versión que mejora la eficiencia de la ejecución. Este procedimiento categoriza las actividades en B y C. Las actividades B son actividades ligadas a la ocurrencia de un suceso y pueden ser programadas con anticipación, mientras que las actividades C se producirán cuando se verifiquen ciertas situaciones o condiciones.

En la Figura 2.6 se muestra un modelo del Diagrama Cíclico de Actividades (Activity Cycle Diagram ó ACD) para un sistema de colas. 
Figura 2.6.

Diagrama Cíclico de Actividades para un sistema de colas.

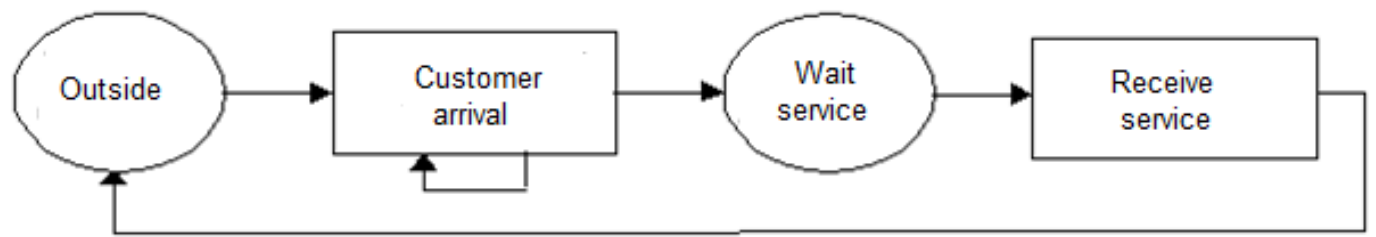

Fuente: Adaptado de Robinson, S. (2004).

- Enfoque de procesos. Muchos modelos de simulación incluyen secuencias de elementos que ocurren en patrones definidos a lo largo del tiempo, por ejemplo, una cola donde las entidades esperan para ser procesadas por el servidor. La lógica asociada con tal secuencia de eventos puede ser generalizada y definida por una única declaración. Luego un lenguaje de simulación puede traducir tales declaraciones en una secuencia de eventos apropiada. Un lenguaje enfocado a los procesos emplea esas declaraciones para modelar el flujo de entidades a través del sistema. Además como una actividad se puede definir como el estado de un objeto entre dos eventos entonces el enfoque por procesos se puede concebir como una sucesión continua de una o más actividades (Vangheluwe, 2001). La mayoría de los programas comerciales de DES trabajan con este enfoque (Heath et. al, 2011) y por lo tanto es el que se usará en este trabajo de grado.

Se deben hacer las siguientes preguntas para iniciar el modelamiento de un sistema por este enfoque:

○ ¿Cuáles son las entidades que se mueven a través del sistema?

○ ¿Qué procesos son ejecutados cuando la entidad se mueve en el sistema?

Un modelo del enfoque por procesos se muestra en la Figura 2.7, se define como un flujograma donde las entidades entran al proceso en el primer paso y abandonan el proceso en el último paso.

Figura 2.7.

Modelo por procesos para un sistema de colas.

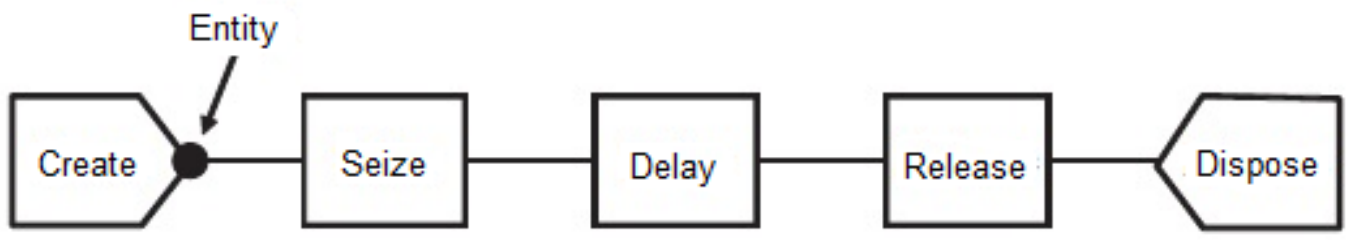

Fuente: Fuente: Kesaraju, V., \& Ciarallo, F. (2012). 
En la Figura 2.8 se representan los tres enfoques para un sistema que consiste en un cajero que atiende a clientes que arriban uno a la vez. Si el cajero no está disponible los clientes se forman en una cola (respetando el orden de llegada). Una vez terminado el servicio los clientes abandonan el sistema.

Figura 2.8.

Sistema de colas con los tres enfoques.

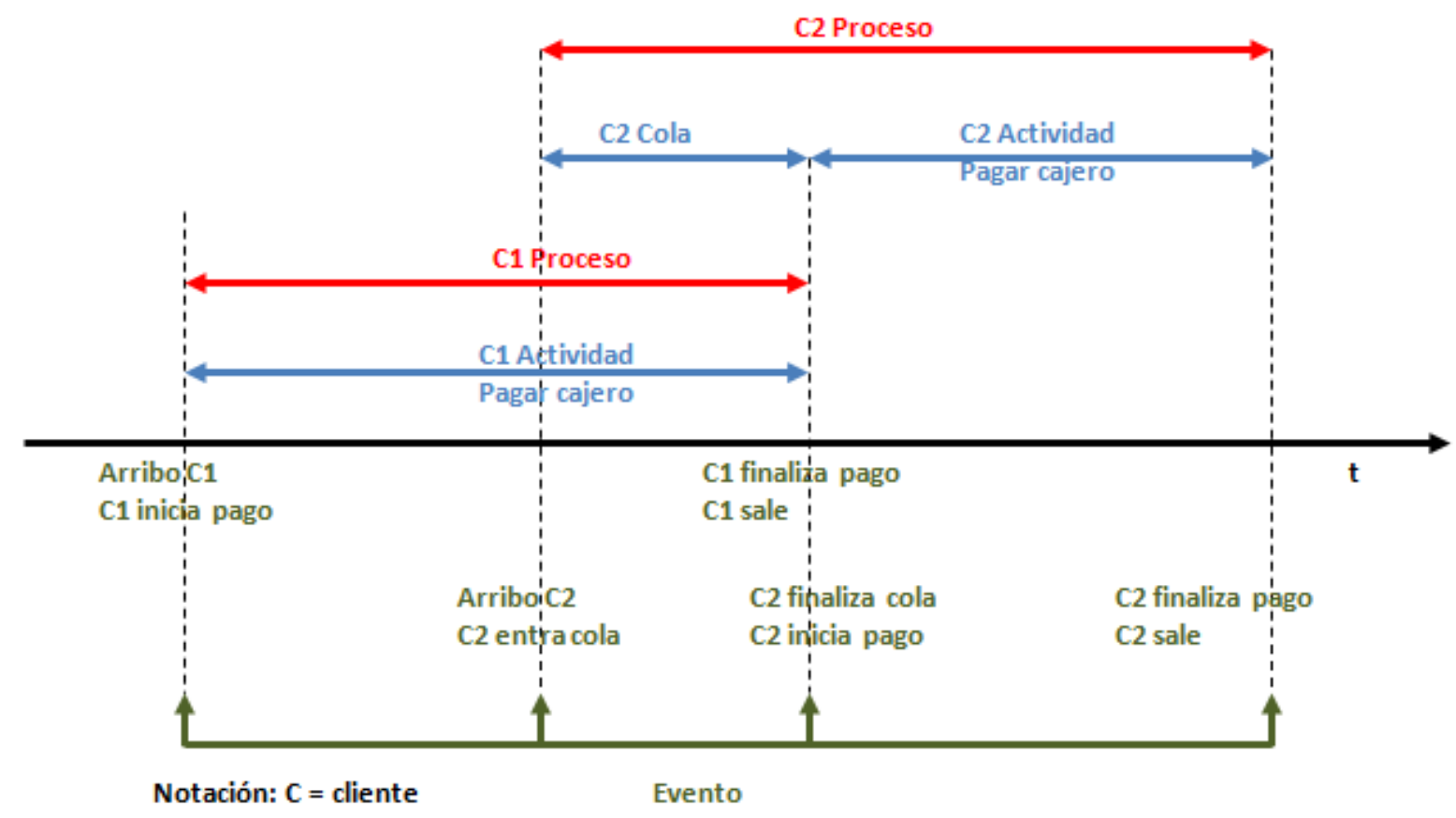

Fuente: Adaptado de Vangheluwe, H. (2001).

Los modelos de DES típicamente tienen componentes estocásticos que imitan la naturaleza probabilística del sistema bajo estudio. Esa aleatoriedad es representada frecuentemente con el uso de variables aleatorias. La obtención de las funciones de probabilidad de dichas variables es lo que se conoce como el análisis de los datos de entrada.

Debido al carácter aleatorio de las variables de entrada del modelo, las medidas de desempeño que se toman al final de la simulación también tienen carácter aleatorio. Es por ello que para que los resultados de la simulación tengan algún significado, se deben usar técnicas estadísticas apropiadas para diseñar y analizar los experimentos (Law, 2014). Por ejemplo, se recomienda hacer inferencias basadas en los intervalos de confianza de las medidas de desempeño más que en estimaciones puntuales de dichas variables. 
A continuación se listan las principales características de DES (Brailsford, 2014b; Marshall et al., 2015a; Robinson, 2014; Skoogh y Johansson, 2008):

- Un elemento clave en casi todos los modelos de DES es la necesidad de representar la aleatoriedad del sistema bajo estudio y por lo tanto se puede decir que DES es estocástica por naturaleza.

- Pertenece a la clasificación de simulación discreta, donde el estado del sistema sólo puede cambiar en puntos discretos de tiempo en los que ocurren eventos.

- Es una metodología de arriba hacia abajo (top-down), es decir, el modelador especifica todas la reglas del sistema con anticipación y se mira desde arriba hacia abajo como se comportan los individuos (o entidades) a lo largo del tiempo. Estas asunciones pueden estar basadas en datos históricos o en opiniones de expertos y en DES están programadas en las actividades. Por lo tanto, las condiciones sobre las que las entidades siguen un ruta en particular o pasan una cierta cantidad de tiempo realizando una actividad tienen que ser codificadas en el modelo.

- Los sistemas son típicamente de bucle abierto (open-loop) donde la retroalimentación no juega un rol preponderante.

- Hacen uso frecuente de animaciones y gráficos, los cuales son muy útiles para comunicarse con los clientes.

- El análisis de los datos de entrada y salida de los modelos de simulación requiere conocimientos de estadística. En un proyecto de DES, la administración de los datos de entrada es a la fase más importante y de mayor consumo de tiempo. Más del $30 \%$ del tiempo del proyecto se usa en la recolección de los datos, transformando los datos en información y suministrando las entradas en el modelo de simulación. Representaciones creíbles de parámetros estocásticos en simulaciones dinámicas requieren un número extenso de datos. Por otro lado, también se requiere el uso de métodos estadísticos para el análisis de los resultados. Se deben tomar decisiones acerca de el número de replicaciones independientes que se requieren, la duración 
de la corrida de simulación, el uso de técnicas de reducción de varianza, la selección de los escenarios, etc.

- Las entidades se consideran objetos pasivos (sin inteligencia o capacidad para tomar decisiones). Estas poseen atributos o características que determinan su comportamiento dentro del sistema y como interactúan con las otras entidades y el entorno. Sin embargo, el término "entidad pasiva" se refiere a que la secuencia de ellas está dominada por reglas globales preestablecidas. Aunque las entidades pueden elegir recorrer ciertos flujos del proceso de acuerdo, por ejemplo, al valor de ciertos atributos esto no elimina el hecho de que siguen las reglas de un sistema centralizado. En otras palabras, no se tiene la facilidad para representar decisiones descentralizadas en DES. Por ejemplo, una vez que una entidad ha empezado a procesarse en una actividad es muy difícil interrumpir el procesamiento basado en cambios del entorno. Justamente esto dificulta modelar la complejidad del comportamiento en ambientes sociales. Como el caso en que las entidades representando a clientes manifiestan su opinión sobre un fabricante o una marca específica y esto influye en la decisión de otros clientes sobre dicho fabricante o marca.

\subsection{Simulación basada en agentes}

La simulación basada en agentes (en inglés "Agent-Based Simulation" y que denotaremos "ABS") fue introducida a principios de los 90s. Aunque en sus inicios este método fue meramente un tópico académico, es durante el periodo 2000-2003 que empezó a ser usado en simulación (Sumari et al., 2013). Entre las razones de su impulso se mencionan:

- El deseo de obtener una visión más profunda de los sistemas cuyo comportamiento no ha sido capturado de forma adecuada por los métodos de modelamiento tradicionales.

- Los avances en la tecnología de modelado procedentes de la informática, como el modelado orientado a objetos, el lenguaje UML (Unified Modeling Language), y los gráficos de estado. 
- El rápido crecimiento de la disponibilidad en las computadoras de CPU y memoria más potentes, ya que los modelos de simulación basados en agentes son más exigentes en cálculos computacionales que los usados en la dinámica de sistemas y en la simulación de eventos discretos.

Los agentes son objetos que tienen las siguientes características (Behdani, 2012):

- Autonomía: los agentes tienen un cierto nivel de autonomía; que les permite tomar decisiones sin un controlador central. Para lograr esto, tienen un conjunto de reglas que determina su comportamiento. Otra forma de tomar decisiones es probabilistamente, usando distribuciones de probabilidad para regir el proceso de decisión.

- Reactividad: los agentes son capaces de percibir los cambios en el entorno (o ambiente) y otros agentes y luego responder a esos cambios con sus propias acciones cuando sea necesario.

- Pro-actividad: los agentes tienen una capacidad proactiva, es decir, que no sólo actúan en respuesta a los cambios que se han producido en su entorno, sino tienen sus propios objetivos.

- Habilidad social: los agentes tienen la capacidad social para interactuar y comunicarse entre sí.

- Adaptabilidad: los agentes pueden tener memoria para aprender y adaptar sus comportamientos basados en la experiencia.

La forma como interactúan los agentes se puede apreciar en la Figura 2.9. 
Figura 2.9.

Interacción entre agentes.

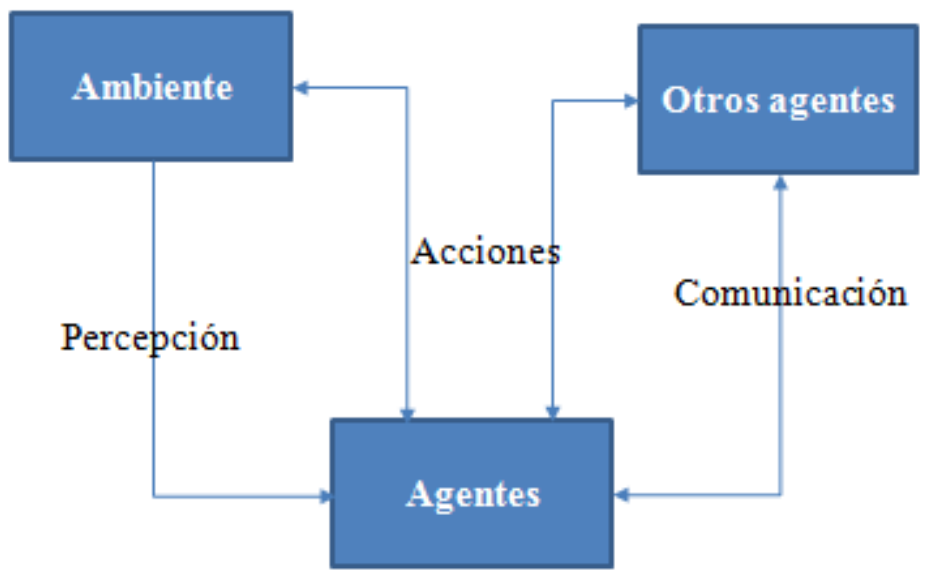

Fuente: Adaptado de Salamon, T. (2011).

Este paradigma sugiere al modelador otra manera de ver el sistema. ABS es muy útil cuando no se conoce como funciona el sistema en su conjunto, o no se puede identificar las variables claves y su interrelación o no se puede distinguir claramente los flujos de proceso. Sin embargo, sí se tiene una idea de cómo los objetos del sistema se comportan de forma individual. Algunas veces se puede dejar que los agentes interactúen entre ellos; otras veces se los puede dejar en un ambiente que tiene su propia dinámica. En cualquiera de los casos, el comportamiento global del sistema emerge de entre muchos (decenas, cientos, miles, incluso millones) comportamientos individuales concurrentes (Borshchev y Filippov, 2004).

A continuación se listan las principales características de ABS (Brailsford, 2014b; Schieritz y Milling, 2003; Macal y North, 2006):

- El principio fundamental en ABS indica que la interacción entre los agentes determina el comportamiento del sistema. Este paradigma se enfoca en las reglas de los agentes, las cuales determinan como se van a dar las interacciones y por lo tanto el comportamiento del sistema como un todo. El componente básico usado en la construcción de un modelo es el agente y se les considera entidades "activas" ya que pueden tomar decisiones autónomas no preestablecidas.

- Es un método de abajo hacia arriba $^{4}$ (bottom-up), es decir, es la fuente de un fenómeno denominado "que emerge". Este fenómeno ocurre cuando las 
interacciones entre objetos en un nivel dan como resultado objetos a otro nivel. En ABS este fenómeno no es modelado previamente como en SD o DES donde se imponen reglas globales al sistema. Por el contrario, en ABS no existen reglas que rijan al sistema sino que el comportamiento de éste emerge a lo largo de la simulación. Lo que sí se debe hacer es describir los comportamientos individuales de los agentes. Estos están basados en asunciones de como los individuos responden a estímulos de otros agentes y del entorno.

- $\mathrm{ABS}$ es una técnica adecuada cuando el problema requiere un fuerte modelamiento en aspectos espaciales o geográficos (posición, tamaño, velocidad, etc.), ya que los agentes son consideradas entidades que pueden interactuar dinámicamente en espacios multidimensionales (1D, 2D o 3D).

- Tradicionalmente los modelos de ABS han hecho un buen uso de los mecanismos de abstracción basados en clases provistos por los lenguajes de programación orientados a objetos. ABS se puede beneficiar del uso de UML para representar los modelos. UML es un leguaje de modelamiento visual para representar sistemas orientados a objetos que comúnmente es adoptado para apoyar los modelos de agentes en las fases de diseño y comunicación. La programación orientada a objetos permite el modelamiento de forma natural de los sistemas de agentes, con el uso de sus clases de objetos como plantillas para los agentes y los métodos de los objetos para representar los comportamientos de los agentes.

- Existen dos métodos para avanzar el reloj en ABS: el método de avance de tiempo de incremento fijo (ó actualización asincrónica) y el método de avance del siguiente evento (ó actualización sincrónica). Tradicionalmente se ha usado el primer método, ya que es simple de implementar (no requiere una programación y una lista eventos) y facilita el modelamiento del comportamiento natural de los agentes ya que no requiere de un coordinador central que lleve la cuenta de cual es el siguiente evento o de una larga cantidad de sincronización entre los agentes. Sin embargo, puede ser inexacto y por lo tanto es clave determinar el tamaño adecuado del incremento. El segundo método, requiere que la duración de las acciones del agente y el entorno sean determinadas con anticipación. Sin embargo, debido a que pueden ocurrir 
muchas interacciones, entonces puede ser necesario cancelar y reprogramar ciertos eventos que ya han sido puestos en la lista de eventos para ocurrir en el futuro. Esto también puede traer ineficiencias en la aplicación del método.

\subsection{Ejemplo comparativo de los paradigmas aplicado a la simulación de tráfico}

A continuación se muestra en la Figura 2.10 un planteamiento de modelamiento del sistema de tráfico a través de una visión asociada con cada uno de los paradigmas.

Figura 2.10.

Paradigmas de simulación en modelamiento de tráfico.

Vista macro: tráfico se modela como un flujo continuo (SD)

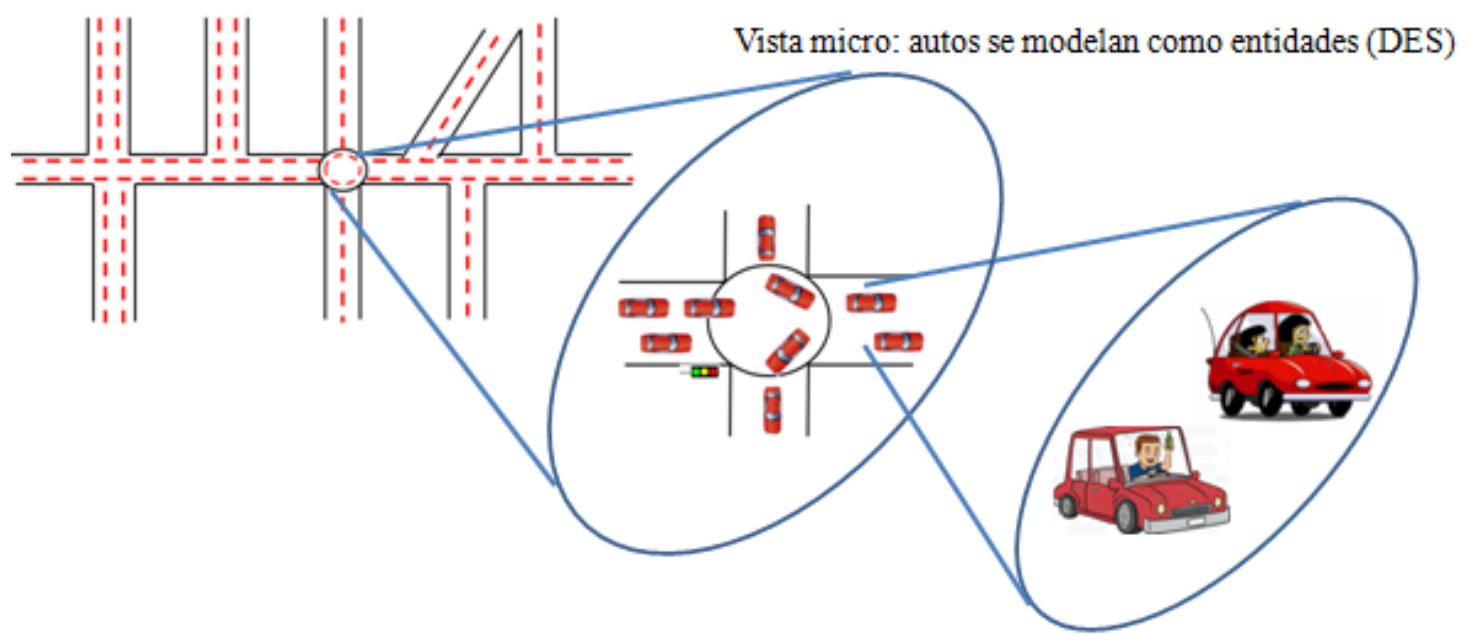

Vista micro: comportamientos de manejo se modelan como reglas de los agentes (ABS)

Elaboración propia.

Es como hacer diferentes escalas de zoom en un mapa. Si lo que se busca, por ejemplo, es estudiar la capacidad de las autopistas (de un área grande) para diferentes niveles de congestión vehicular entonces se puede ver el tráfico como un flujo de automóviles, similar a un fluido moviéndose en un conjunto de tuberías. Esta visión encaja con el paradigma de SD. Aspectos importantes a considerar son la densidad de vehículos y su velocidad media. Por otro lado, si lo que se busca es controlar el tráfico en un área más focalizada, como por ejemplo un óvalo vehicular, entonces esto se puede hacer a través del control de la frecuencia del cambio de luces (fija o variable con la ayuda de cámaras) o si es necesario colocar nuevos semáforos en las entradas al óvalo. A este nivel se requiere representar los carros de forma individual con el fin de mostrar las colas y los tiempos promedios de espera de los autos. El paradigma de DES 
es el adecuado en este caso. Finalmente, si lo que se quiere es modelar el comportamiento de los conductores y como esas características peculiares de manejo influyen sobre los otros conductores y en el tráfico en general entonces se requiere representar a los autos como agentes y a las formas de manejo como reglas de interacción. El paradigma adecuado en este caso es ABS. 


\subsection{Cuadro comparativo de los paradigmas}

La Tabla 2.1 muestra una recolección de los elementos distintivos de cada paradigma expuestos por diversos autores (Marshall et al., 2015b; Behdani, 2012; Tacko y Robinson, 2009):

Tabla 2.1.

Características de los paradigmas de simulación.

\begin{tabular}{|c|c|c|c|}
\hline Aspecto & Dinámica de Sistemas (SD) & $\begin{array}{l}\text { Simulación de Eventos } \\
\text { Discretos (DES) }\end{array}$ & $\begin{array}{l}\text { Simulación Basada en Agentes } \\
\text { (ABS) }\end{array}$ \\
\hline Visión del modelo & $\begin{array}{l}\text { Sucesión de niveles y tasas } \\
\text { interconectados por relaciones } \\
\text { casuales }\end{array}$ & $\begin{array}{l}\text { Entidades fluyendo a través de } \\
\text { una red de actividades y colas }\end{array}$ & $\begin{array}{l}\text { Conjunto de agentes } \\
\text { interactuando entre ellos y el } \\
\text { entorno }\end{array}$ \\
\hline $\begin{array}{l}\text { Perspectiva del } \\
\text { comportamiento del } \\
\text { sistema }\end{array}$ & $\begin{array}{l}\text { De arriba hacia abajo; reglas } \\
\text { globales de comportamiento; por } \\
\text { ejemplo la tasa de compra de un } \\
\text { producto en unidades por mes }\end{array}$ & $\begin{array}{l}\text { De arriba hacia abajo; predefine } \\
\text { comportamiento global de las } \\
\text { entidades; por ejemplo, el } \\
\text { estipular que el } 30 \% \text { de los } \\
\text { artículos son defectuosos }\end{array}$ & $\begin{array}{l}\text { De abajo hacia arriba; } \\
\text { comportamiento global no es } \\
\text { predefinido sino que emerge por la } \\
\text { interacción de los agentes } \\
\text { (comportamiento individual) }\end{array}$ \\
\hline $\begin{array}{l}\text { Causante del } \\
\text { comportamiento dinámico } \\
\text { del sistema }\end{array}$ & $\begin{array}{l}\text { Los bucles de realimentación; } \\
\text { la estructura del sistema } \\
\text { determina su comportamiento a } \\
\text { través del tiempo }\end{array}$ & $\begin{array}{l}\text { La ocurrencia de los eventos; } \\
\text { éstos cambian el estado del } \\
\text { sistema }\end{array}$ & $\begin{array}{l}\text { La interacciones entre los } \\
\text { agentes; simples reglas en cada } \\
\text { agente dan origen al } \\
\text { comportamiento colectivo }\end{array}$ \\
\hline Estructura del sistema & $\begin{array}{l}\text { La estructura del sistema es } \\
\text { fija; no se pueden añadir nuevos } \\
\text { niveles o flujos ni conectar } \\
\text { nuevas relaciones causales } \\
\text { durante la simulación }\end{array}$ & $\begin{array}{l}\text { El flujograma del proceso es } \\
\text { fijo; durante la simulación no se } \\
\text { pueden agregar nuevas } \\
\text { actividades o cambiar las reglas } \\
\text { de decisión que ya han sido } \\
\text { preestablecidas }\end{array}$ & $\begin{array}{l}\text { La estructura del sistema no es } \\
\text { fija; cambia a lo largo del tiempo, } \\
\text { donde nuevos agentes pueden ser } \\
\text { creados y sus interrelaciones } \\
\text { modificadas durante la simulación } \\
\text { permitiendo que los agentes sigan } \\
\text { cursos de acción distintos a los } \\
\text { planteados originalmente }\end{array}$ \\
\hline
\end{tabular}




\begin{tabular}{|c|c|c|c|}
\hline Aspecto & Dinámica de Sistemas (SD) & $\begin{array}{l}\text { Simulación de Eventos } \\
\text { Discretos (DES) }\end{array}$ & $\begin{array}{l}\text { Simulación Basada en Agentes } \\
\text { (ABS) }\end{array}$ \\
\hline Resolución & $\begin{array}{l}\text { Nivel macro: entidades } \\
\text { agrupadas y homogéneas; se } \\
\text { asume que todas las entidades } \\
\text { tienen características similares; } \\
\text { se trabaja con valores promedio. } \\
\text { Por ejemplo, los autos en una } \\
\text { autopista, sin diferenciarlos por } \\
\text { marca o tamaño. }\end{array}$ & $\begin{array}{l}\text { Nivel micro: entidades } \\
\text { individuales (pasivas) y } \\
\text { heterogéneas; por ejemplo, } \\
\text { productos distintos en un proceso } \\
\text { productivo. }\end{array}$ & $\begin{array}{l}\text { Nivel micro: entidades } \\
\text { individuales (activas) y } \\
\text { heterogéneas; por ejemplo, una } \\
\text { cadena de suministro donde los } \\
\text { agentes representan a los } \\
\text { eslabones de la cadena: clientes, } \\
\text { productores, proveedores, y cada } \\
\text { uno con sus propios objetivos. }\end{array}$ \\
\hline Naturaleza de los datos & $\begin{array}{l}\text { Determinístico; los mismos } \\
\text { datos de entrada generan los } \\
\text { mismos resultados }\end{array}$ & $\begin{array}{l}\text { Estocástico; trabaja con variables } \\
\text { aleatorias; análisis de resultados } \\
\text { requiere replicaciones } \\
\text { independientes }\end{array}$ & $\begin{array}{l}\text { Estocástico; mayormente las } \\
\text { reglas de decisión están basadas en } \\
\text { funciones de probabilidad. }\end{array}$ \\
\hline Manejo del tiempo & $\begin{array}{l}\text { Continuo; utiliza el método de } \\
\text { avance del tiempo por } \\
\text { incremento fijo }\end{array}$ & $\begin{array}{l}\text { Discreto; utiliza el método de } \\
\text { avance de tiempo del siguiente } \\
\text { evento }\end{array}$ & $\begin{array}{l}\text { Continuo/Discreto; puede } \\
\text { implementarse en un modelo } \\
\text { cualquiera de los dos métodos de } \\
\text { avance de tiempo: por incremento } \\
\text { fijo ó del siguiente evento }\end{array}$ \\
\hline Formulación matemática & $\begin{array}{l}\text { Ecuaciones diferenciales de } \\
\text { primer orden; éstas representan } \\
\text { a las tasas y los niveles acumulan } \\
\text { las tasas mediantes ecuaciones de } \\
\text { integración }\end{array}$ & $\begin{array}{l}\text { Combinación de expresiones } \\
\text { matemáticas con operadores } \\
\text { lógicos }\end{array}$ & $\begin{array}{l}\text { Combinación de expresiones } \\
\text { matemáticas con operadores } \\
\text { lógicos y reglas de decisión }\end{array}$ \\
\hline $\begin{array}{l}\text { Dependencia de la } \\
\text { trayectoria }\end{array}$ & $\begin{array}{l}\text { Estados futuros del sistema } \\
\text { dependen sólo del estado } \\
\text { actual; el valor de una variable } \\
\text { en el instante "t+1" depende de } \\
\text { los valores de otras variables en } \\
\text { el instante actual "t" }\end{array}$ & $\begin{array}{l}\text { Estados futuros del sistema } \\
\text { dependen sólo del estado } \\
\text { actual; la actualización de una } \\
\text { variable en el siguiente evento } \\
\text { sólo depende del valor que tenía } \\
\text { en el evento anterior }\end{array}$ & $\begin{array}{l}\text { Estados futuros del sistema } \\
\text { dependen del estado actual y de } \\
\text { otros estados anteriores y } \\
\text { decisiones previas; los agentes } \\
\text { pueden poseer en su memoria } \\
\text { interna la recolección de varios }\end{array}$ \\
\hline
\end{tabular}




\begin{tabular}{|l|l|l|l|}
\hline Aspecto & Dinámica de Sistemas (SD) & $\begin{array}{l}\text { Simulación de Eventos } \\
\text { Discretos (DES) }\end{array}$ & $\begin{array}{l}\text { Simulación Basada en Agentes } \\
\text { (ABS) }\end{array}$ \\
\hline Enfoque jerárquico & $\begin{array}{l}\text { Estratégico; donde es más } \\
\text { otros agentes) y que influyan en } \\
\text { sus decisiones futuras }\end{array}$ \\
\hline $\begin{array}{l}\text { común encontrar problemas más } \\
\text { determinística producto de } \\
\text { políticas }\end{array}$ & $\begin{array}{l}\text { Operacional; donde es más } \\
\text { común encontrar problemas } \\
\text { asociados con efectos aleatorios } \\
\text { interconectados }\end{array}$ & $\begin{array}{l}\text { Operacional/Estratégico. En el } \\
\text { caso estratégico, por ejemplo, los } \\
\text { agentes pueden representar a } \\
\text { compañías compitiendo por un } \\
\text { nuevo mercado. } \\
\text { En el caso operacional dos agentes } \\
\text { pueden representar, uno a un } \\
\text { operario y el otro a una máquina. }\end{array}$ \\
\hline
\end{tabular}

Elaboración propia. 


\section{CAPÍTULO III: CASO DE ESTUDIO: E-BIKE SAN REMO}

Este es un caso de estudio ficticio, elaborado con el fin de facilitar la comparación de forma cuantitativa de los paradigmas de simulación.

\subsection{La compañía}

E-bike San Remo (ESR) se dedica a fabricar bicicletas eléctricas. Fue fundada por Roque Molina, un ingeniero electrónico que practicó activamente el ciclismo en los años 80's, llegando a participar en varias competiciones internacionales. Su pasión por el ciclismo es compartida por su dedicación a la electrónica. Justamente esta mezcla de intereses y su visión de que los medios de transporte con energías sostenibles y renovables se irán popularizando en el mercado peruano, es que lo llevó a fundar ESR. La compañía opera como una empresa familiar ubicada en la ciudad de Arequipa-Perú. Roque, su hermano Juan y dos operarios más trabajan en la elaboración de las bicicletas, mientras que su esposa Clara se dedica a la manejar la parte administrativa.

\subsection{El producto y el proceso de fabricación}

Una bicicleta eléctrica es una bicicleta que tiene acoplado un motor eléctrico. Se suministra energía al motor eléctrico por medio de la batería y el motor facilita la función mecánica del pedaleo lo que se conoce como sistema de pedaleo asistido. Al presionar el pedal o al dar vueltas el plato, un controlador electrónico registra esta señal de un sensor y proporciona la energía al motor para que éste asista al avance de la bicicleta a una velocidad y fuerza calculadas. Cuando se deja de pedalear o se acciona el freno, el motor deja de funcionar.

El modelo de bicicleta vendido por ESR se denomina VELOZ y se basa en un diseño ergonómico del cuadro (o marco) que además incluye de una manera eficiente los requerimientos de espacio impuestos por la batería y el motor. Este cuadro es fabricado por ESR con tubos de titanio que se juntan mediante soldadura. El titanio es ligeramente más elástico que el acero, permitiendo absorber las vibraciones de la carretera antes de que lleguen al ciclista. Además es un material que no se oxida y no 
necesita anticorrosivos. Los demás componentes como el motor, la batería, los sensores de movimiento, los neumáticos, etc. se compran de fabricantes de alta calidad y se ensamblan en las instalaciones de ESR. En la Figura 3.1 se muestra la ilustración del modelo VELOZ detallando sus partes más importantes.

El tiempo de producción de una bicicleta VELOZ, es decir, desde que se empieza a trabajar la orden de producción hasta que se deja la bicicleta en el inventario de productos terminados sigue la siguiente distribución de probabilidad: $2+\operatorname{NORM}(1,0.5)$ días. Donde el efecto aleatorio se encuentra en el segundo término indicado por la distribución normal con media de 1 día y desviación estándar de 0.5 días. Por lo tanto, toma en promedio 3 días para fabricar una bicicleta VELOZ. Este tiempo de fabricación es considerando 1 turno de 8 horas diarias.

Figura 3.1.

Ilustración del modelo VELOZ.

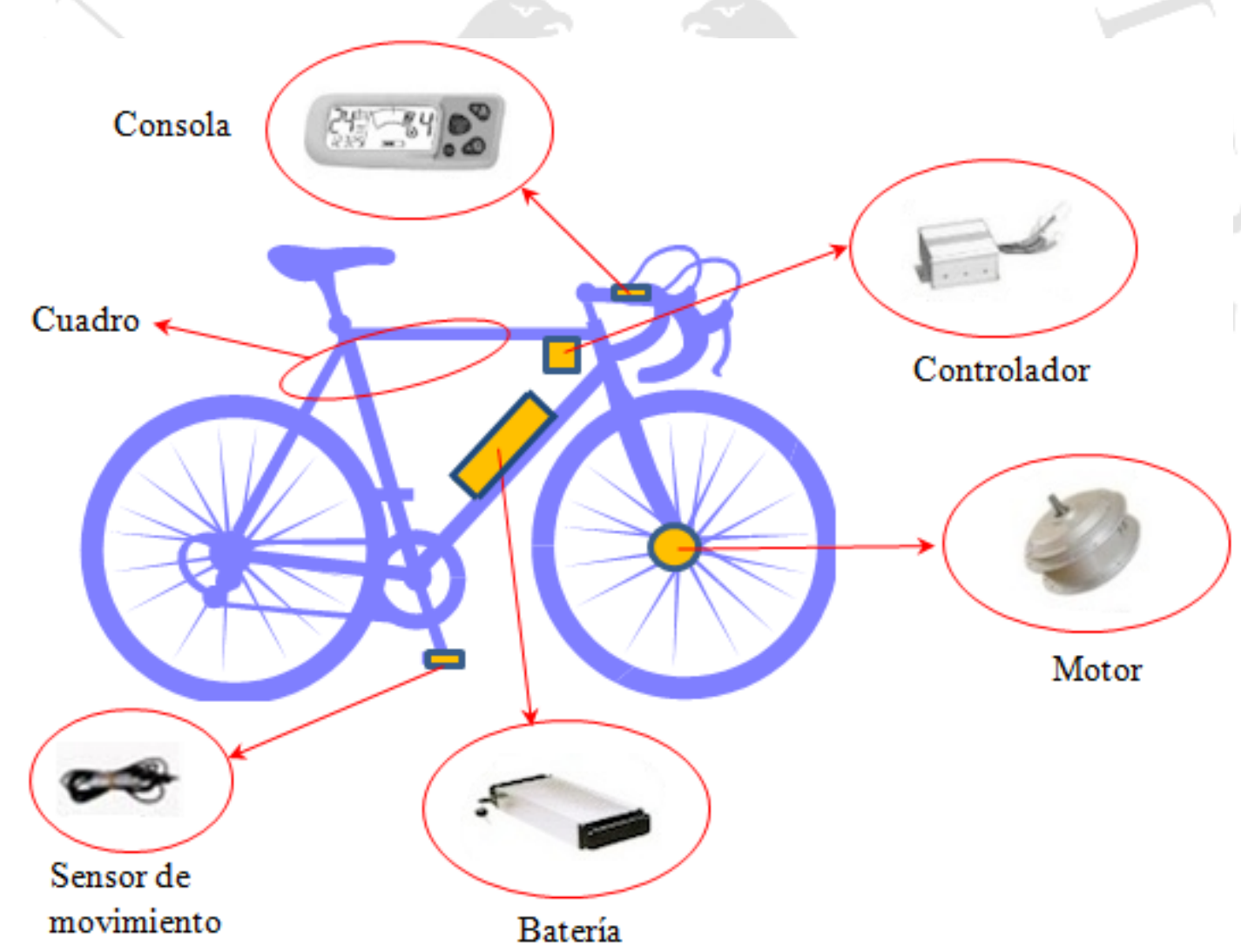

Fuente:Adaptado de Electric Bikes Utah (2015).

Existen dos líneas de producción. En cada línea se trabajan las tres etapas del proceso de fabricación. La primera etapa es la fabricación del marco, la segunda etapa es el ensamble de los componentes y finalmente la tercera etapa es el control de calidad. Roque y un operario trabajan en una de las líneas y Juan con el otro operario trabajan en 
la segunda línea. De esa forma se puede cumplir (en cada línea) con el tiempo de producción de una bicicleta definido anteriormente.

La fabricación del marco se inicia cuando llega la orden de producción, es decir, no se produce para inventario. Se asume que una orden de producción es sólo por una bicicleta. El resto de componentes se compran en tiendas locales o pueden ser enviados desde Lima, en este último caso, con un tiempo máximo de entrega de 1 día. Esto implica que estos componentes ya están listos para su uso cuando se termina de fabricar el marco. Además, se mantiene un inventario de partes pequeñas como pedales, frenos o cadenas.

\subsection{El pronóstico de ventas}

\subsubsection{Modelo de difusión tecnológica}

Se utilizará el modelo de Bass (1969) para representar las ventas de las bicicletas eléctricas. Este modelo permite estimar el número de consumidores que adquieren (adoptan) $)^{5}$ un nuevo producto a lo largo del tiempo. Existe un mercado potencial conformado por todas aquellas personas que tienen acceso a la información sobre el producto nuevo y además tienen la capacidad para adquirirlo (compradores potenciales). La adquisición o compra del producto se ve influenciada por dos tipos de factores:

a) Factor de influencia externa, donde la información que llega a los compradores potenciales sobre el nuevo producto proviene de fuentes externas como los medios masivos de comunicación.

b) Factor de influencia interna, donde la información del producto nuevo llega por una interacción entre los compradores potenciales y los que ya han adquirido el producto (compradores). Este es un proceso de imitación originado por la interacción de "boca a boca". Dichas interacciones no tienen que ocurrir necesariamente de forma física, pueden ser por teléfono, e-mail, etc.

La población objetivo representa el número máximo de consumidores que pueden adquirir el producto. Esto no significa toda la población del país o región bajo análisis, sino sólo los compradores potenciales. En este modelo el tamaño de la

\footnotetext{
En este documento se usan como equivalentes los términos: "comprar un producto" y "adoptar un producto"
} 
población objetivo se considera constante a lo largo del tiempo, es decir, la población objetivo está conformada por la suma de los consumidores potenciales y los compradores. Por lo tanto, al inicio, el mercado potencial es igual a la población objetivo y luego la innovación va penetrando dicho mercado, a través de las influencias interna y externa. El proceso de adopción se inicia cuando los primeros compradores toman conciencia de la innovación a través de fuentes externas de información como son los medios de comunicación. Luego, la innovación se difunde también a través del boca a boca, donde los que ya han adquirido el producto se ponen en contacto con los que no lo tienen exponiéndolos con la idea o el deseo de comprar el producto nuevo De allí saldrán nuevos adeptos que persuadirán a terceros y así sucesivamente hasta la saturación de la población objetivo. En ese momento, ya no hay más compradores potenciales y todas las personas de la población han adquirido el producto.

Se usará la siguiente notación para representar a las variables y parámetros del modelo:

M: tamaño de la población objetivo

$\mathrm{C}_{\mathrm{t}}$ : número acumulado de compradores en el tiempo $\mathrm{t}$

$\mathrm{P}_{\mathrm{t}}$ : número total de compradores potenciales en el tiempo $\mathrm{t}$.

Por lo expresado anteriormente se cumple que $\mathrm{M}=\mathrm{P}_{\mathrm{t}}+\mathrm{C}_{\mathrm{t}}$

La tasa de adopción (TA) o compra del producto nuevo se expresa en personas/unidad de tiempo. Se calcula sumando la tasa de adopción por la publicidad (factor externo) y la tasa de adopción por el boca a boca (factor interno).

$\mathrm{TA}_{\mathrm{t}}=\mathrm{TA}$ por Publicidad $t+\mathrm{TA}$ por Boca a Boca $\mathrm{t}$

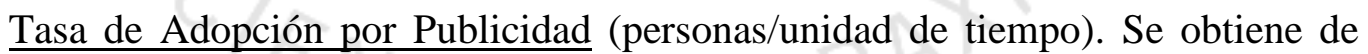
multiplicar el número de compradores potenciales por una Fracción de Efectividad debido a la campaña publicitaria. Esta fracción representa la proporción o el porcentaje de los compradores potenciales que adquieren el producto en un periodo de tiempo dado. Por ejemplo, una Fracción de Efectividad de 0.1 semanal indica que cada semana el 10 por ciento de los compradores potenciales adquieren el producto por influencia de los medios de comunicación.

TA por Publicidad ${ }_{\mathrm{t}}=\mathrm{P}_{\mathrm{t}} *$ Fracción de Efectividad 
Por ejemplo, en la semana t para $P_{t}=40$ y Fracción de Efectividad=0.1 semanal entonces

TA por Publicidad $_{\mathrm{t}}=40 * 0.1=4$ personas por semana (compran el producto)

Tasa de Adopción por el Boca a Boca (personas/unidad de tiempo). Se calcula multiplicado los siguientes cuatro factores: a) número de compradores, b) tasa de contacto c) probabilidad de un contacto favorable y d) fracción de adopción (Morecroft, 2007).

La tasa de contacto representa la tendencia a socializar, indicando el número de personas promedio que un individuo puede contactar para comentar sobre el producto nuevo en un periodo de tiempo. Por ejemplo, una tasa de contacto de 20 semanal indica que una persona está en contacto en una semana con un promedio con 20 personas a las cuales les comentó sobre el producto nuevo.

La probabilidad de un contacto favorable indica la probabilidad de que un contacto aleatorio entre dos personas se de entre un comprador potencial y un comprador. Se asume que un contacto entre dos compradores o un contacto entre dos compradores potenciales no genera una adquisición del producto. Para cada comprador existen $\mathrm{P}_{\mathrm{t}}$ compradores potenciales a los cuales se puede contactar en un tiempo determinado. Por lo tanto, para cada comprador la probabilidad de un contacto favorable se define como $\mathrm{P}_{\mathrm{t}} / \mathrm{M}$.

No todo contacto favorable implica que el producto va a ser comprado. La fracción de adopción representa la proporción o el porcentaje de contactos favorables que sí terminaron en la adquisición del producto. Esto se da por parte de los que eran compradores potenciales y que se convierten ahora en compradores reales. Por ejemplo, una Fracción de Adopción de 0.05 ó 5\% indica que de 100 contactos favorables sólo 5 de ellos terminan en compras del producto.

TA por Boca a Boca $\mathrm{t}_{\mathrm{t}}=\mathrm{C}_{\mathrm{t}} *$ Tasa de Contacto $* \mathrm{P}_{\mathrm{t}} / \mathrm{M} *$ Fracción de Adopción

Por ejemplo, en la semana $\mathrm{t}$ para $\mathrm{C}_{\mathrm{t}}=60, \mathrm{P}_{\mathrm{t}}=40, \mathrm{M}=100$, Tasa de Contacto $=5$ semanal y Fracción de Adopción=0.05 entonces

TA por Boca a Boca $=60 * 5 * 40 / 100 * 0.05=6$ personas por semana (compran el producto) 
Finalmente para completar el ejemplo, la Tasa de Adopción del producto en la semana t sería la suma de los dos tasas anteriores:

$\mathrm{TA}_{\mathrm{t}}=4+6=10$ personas por semana (compran el producto)

El número acumulado de compradores en el tiempo t estará dado por:

$\mathrm{C}_{\mathrm{t}}=\int_{0}^{\mathrm{t}} \mathrm{TA}_{\mathrm{s}} \mathrm{ds}$

Además se cumple que: Límite ${ }_{t \rightarrow \infty} \mathrm{C}_{\mathrm{t}}=\mathrm{M}$

Otra forma de expresar $\mathrm{C}_{\mathrm{t}}$ es la siguiente:

$\mathrm{C}_{\mathrm{t}}=\mathrm{X}_{\mathrm{t}}+\mathrm{C}_{\mathrm{t}-1}, \mathrm{t} \geq 1$

donde $\mathrm{X}_{\mathrm{t}}$ representa el número de compradores que han adquirido el producto en el tiempo t y se cumple que: $\mathrm{X}_{\mathrm{t}}=\mathrm{TA}_{\mathrm{t}} \Delta \mathrm{t}$.

La Figura 3.2 muestra como cambia a lo largo del tiempo la Tasa de Adopción del modelo de Bass y sus componentes la Tasa de Adopción por Publicidad y la Tasa de Adopción por Boca a Boca. La forma de una curva de campana de la Tasa de Adopción viene influenciada por la Tasa de Adopción por Boca a Boca. La Tasa de Adopción por Publicidad influye en el punto de partida de dicha curva de campana.

Figura 3.2.

Modelo de Bass.

\section{Tasa de Adopción}

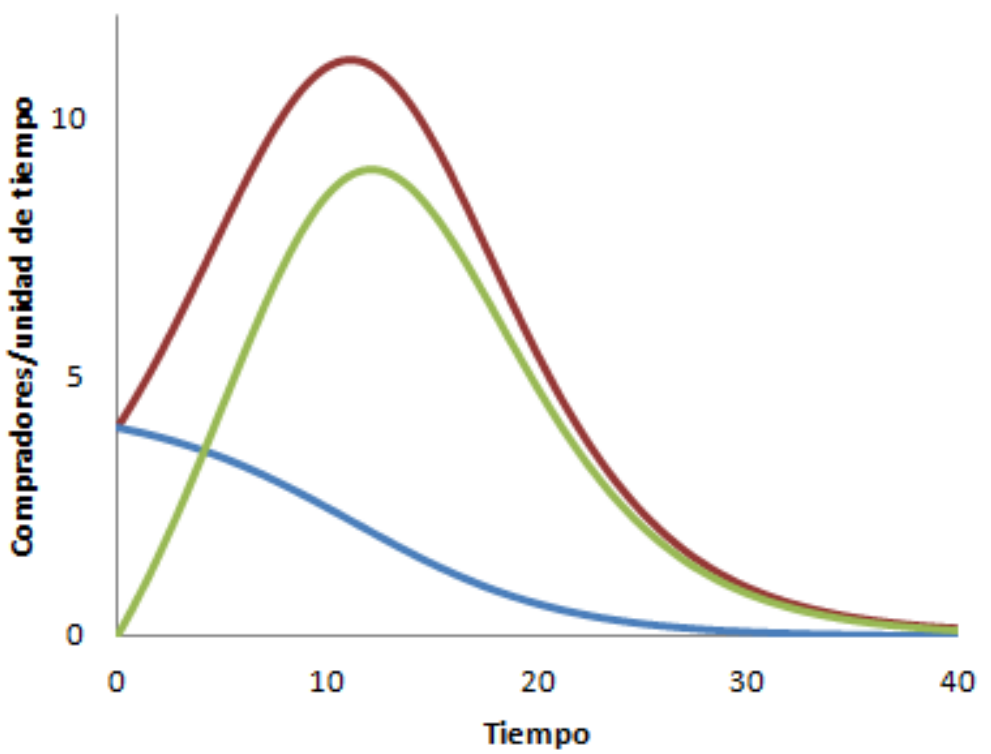

- Tasa de Adopción

Tasa de Adopción por Publicidad

- Tasa de Adopción por Boca a Boca

Elaboración propia. 


\subsubsection{Segmento de mercado y parámetros del modelo}

El mercado o población objetivo de la empresa San Remo está conformado por personas de la ciudad de Arequipa amantes del ciclismo y que además estarían dispuestos a pagar 2000 dólares por una bicicleta eléctrica de muy alta calidad. Estas personas participan continuamente en actividades de ciclismo, son miembros activos de asociaciones de ciclistas y de transporte ecológico y que en muchos de los casos utilizan la bicicleta como un medio de transporte rutinario.

Se ha identificado un mercado potencial en Arequipa de 200 personas, es decir, M=200. El resto de los parámetros del modelo de Bass quedan definidos de la siguiente manera:

Fracción de Efectividad por la publicidad $=0.01$ ó $1 \%$ quincenal

Tasa de Contacto $=8$ personas por quincena

Fracción de Adopción = 0.045 ó 4.5\%

Estos valores se estimaron basándose en datos de estudios donde se aplicó el modelo de Bass para determinar la demanda de bicicletas eléctricas en pequeñas ciudades europeas. Luego estos datos se ajustaron a la realidad de una ciudad como Arequipa con una población de alrededor de 800 mil habitantes. El esquema de este modelo de negocio se muestra en la Figura 3.3.

Figura 3.3.

Esquema del modelo de negocio de ESR.

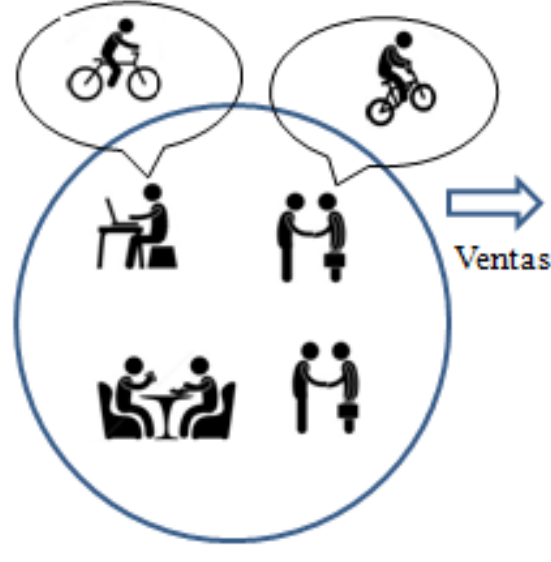

Pronóstico de ventas

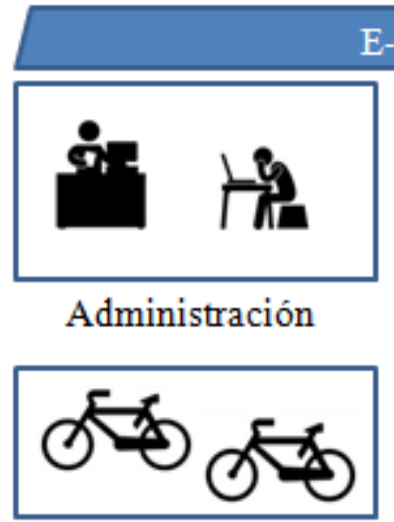

Almacén de PT

\section{E-Bike San Remo}
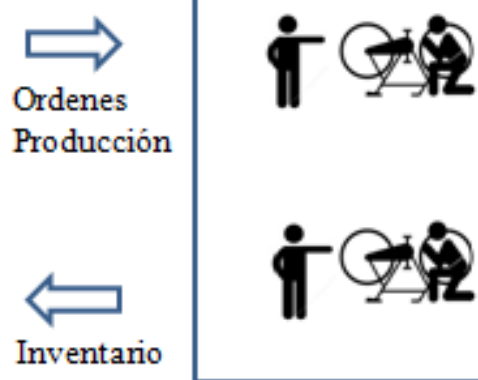

Líneas de producción

Elaboración propia. 
Adicionalmente a los parámetros del modelo de Bass se considera un tasa de producción de la bicicleta modelo VELOZ de 10 bicicletas por quincena. Eso se logra trabajando dos líneas de producción, donde cada línea puede fabricar 5 bicicletas quincenales. Es decir, 5 bicicletas en 15 días equivale a 3 días por bicicleta, que fue el valor promedio indicado en la sección 3.2.

\subsection{Modelo de simulación de eventos discretos}

Una versión estocástica del modelo de Bass descrito en la sección 3.3 se puede expresar haciendo que el número de compradores en el tiempo t sea una variable aleatoria. Por lo tanto,

$C_{t}=X_{t}+C_{t-1}, t \geq 1$

Siendo en este caso $X_{t}$ una variable aleatoria con una distribución de Poisson, es decir,

$P\left(X_{t}=x_{t}\right)=e^{-\lambda_{t}} \frac{\lambda_{t}^{x_{t}}}{x_{t} !}$

donde $\lambda_{\mathrm{t}}=\mathrm{TA}_{\mathrm{t}}=\mathrm{TA}$ por Publicidad $t+\mathrm{TA}$ por Boca a Boca $\mathrm{t}$

Para representar este modelo de Bass en DES se usará una forma equivalente:

Si $X_{t}$ es una variable aleatoria de Poisson con parámetro $\lambda_{t}$, que describe el número de compradores por unidad de tiempo t, entonces la variable aleatoria $Y_{t}$ que describe el tiempo entre dos compras consecutivas ocurridas en los instantes t y t-1 tiene una distribución exponencial con parámetro $\beta_{\mathrm{t}}=1 / \lambda_{\mathrm{t}}$, es decir,

$\mathrm{P}\left(\mathrm{Y}_{\mathrm{t}} \leq \mathrm{y}_{\mathrm{t}}\right)=1-\mathrm{e}^{-\beta_{\mathrm{t}} \mathrm{y}_{\mathrm{t}}}$

En la Figura 3.4 se muestra el modelo de negocio de ESR (pronóstico de ventas y producción de la bicicleta) usando el software ARENA que trabaja el enfoque de DES por procesos (Rockwell Software Inc., 2010).

El modelo de Bass requiere inicializar la Tasa de Adopción en el instante $\mathrm{t}=0$. Esto se logra haciendo que la Tasa de Adopción para $\mathrm{t}=0$ sea igual al Tasa de Adopción por Publicidad, es decir, $\mathrm{TA}_{\mathrm{t}=0}=\mathrm{TA}$ por Publicidad $\mathrm{t}_{\mathrm{t}=0}=\mathrm{P}_{\mathrm{t}=0} *$ Fracción de Efectividad. Ya que el valor de la Tasa de Adopción por el Boca a Boca en $t=0$ es cero. Esto es debido a que $\mathrm{P}_{\mathrm{t}=0}=200$ y $\mathrm{C}_{\mathrm{t}=0}=0$, éste último factor hace que el producto de los términos sea cero. 
En ARENA se crea una entidad ficticia en el instante cero con el único propósito de inicializar la Tasa de Adopción e inmediatamente ser eliminada del sistema.

Figura 3.4.

Modelo de negocio de ESR en ARENA.
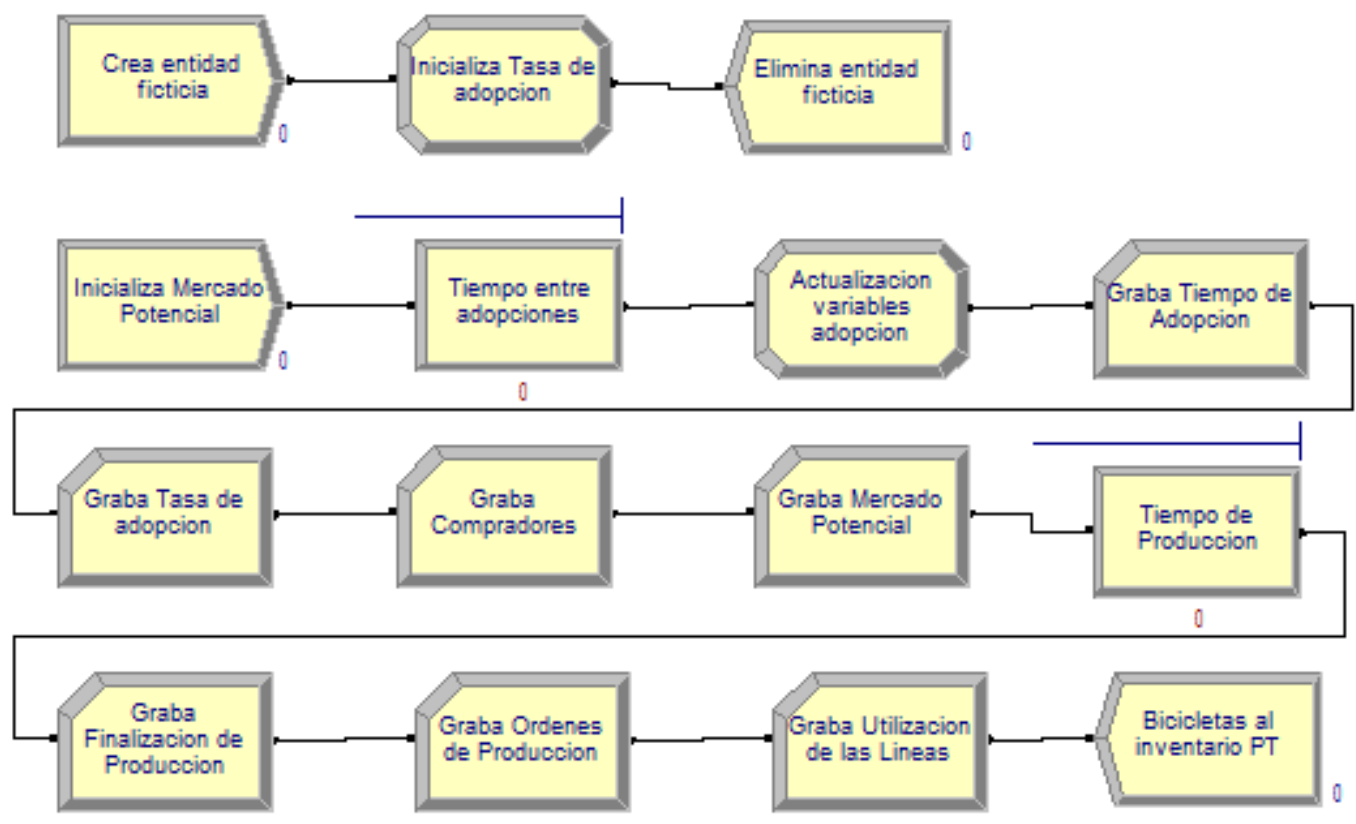

Elaboración propia.

El Mercado Potencial se inicializa haciendo una sola creación de 200 entidades en $\mathrm{t}=0$ correspondientes al tamaño de la población objetivo. Luego se van determinando las nuevas adopciones del producto, una a una, representadas por entidades distanciadas por un tiempo exponencial entre adopciones EXPO(1/Tasa Adopción). La Tasa de Adopción es variable y se actualiza cada vez que ocurre una nueva adopción. En ese momento también se graban en un archivo de Excel las estadísticas de las variables: Tiempo de Adopción, Tasa de Adopción, Compradores y Mercado potencial.

Finalmente, cada adopción se convierte en una orden de producción de una bicicleta, la cual es fabricada en - un tiempo $2+\operatorname{NORM}(1,0.5)$ días ó $(2+\operatorname{NORM}(1,0.5)) / 15$ quincenas. El valor de la variable Ordenes de Producción representa las órdenes que están en espera de fabricación, es decir, el número de entidades en cola en espera de ser procesadas en la producción. Luego se graban en un archivo de Excel las estadísticas de las variables: Finalización de Producción, Ordenes de Producción y la medida de desempeño Porcentaje de Utilización de Líneas (de producción) y la entidad deja el sistema rumbo al inventario de productos terminados. 
El código del modelo en el lenguaje de simulación SIMAN, en el cual se basa el software ARENA, se muestra en el Anexo 1.

Se corrieron 1000 replicaciones del modelo y a continuación se muestran gráficamente los resultados de algunas de las replicaciones de la simulación. La Figura 3.5 muestra 3 pares de trayectorias de la difusión de la bicicleta en el mercado, obtenidas en la replicaciones 6, 20 y 1 . Cada par está conformado por dos curvas del mismo color: a) una curva ascendente que indica el número acumulado de compradores (línea continua) y b) una curva descendente que representa el cambio en el tamaño del mercado potencial (compradores potenciales) mostrada como una línea punteada.

Figura 3.5.

Resultados de tres replicaciones de Compradores y Mercado Potencial.

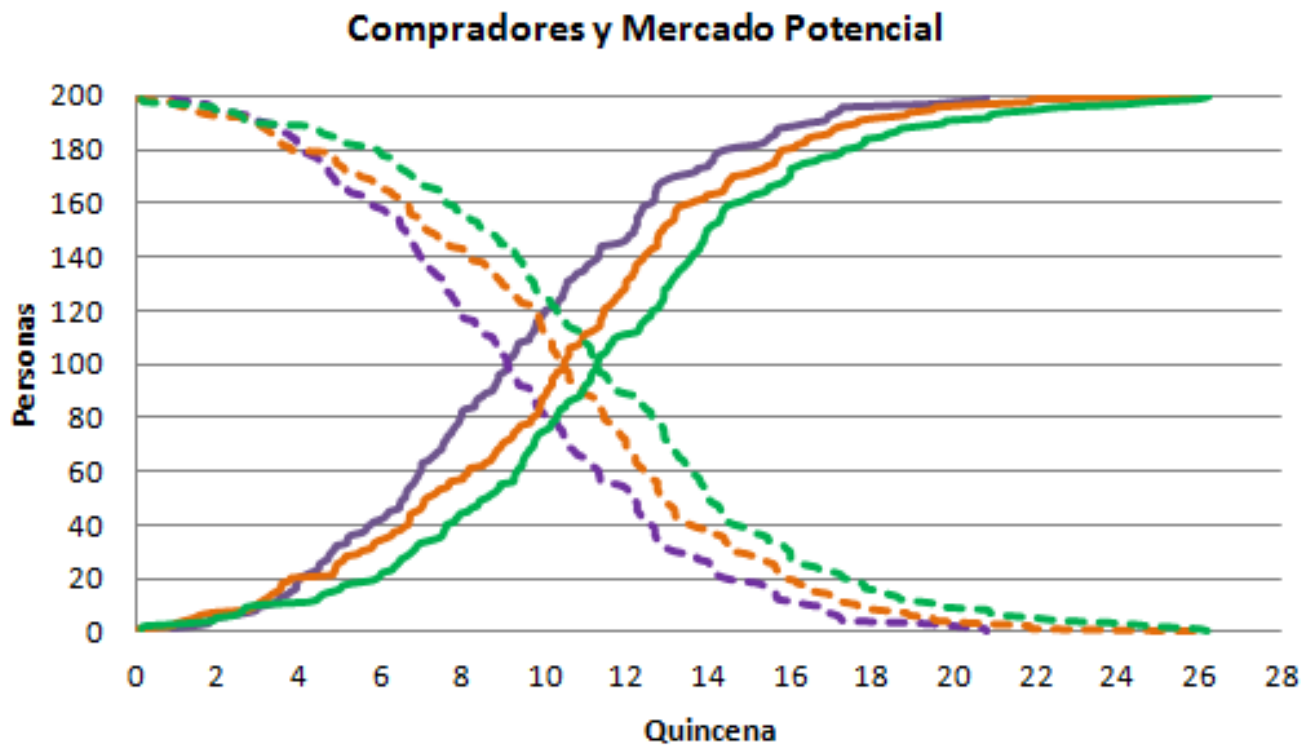

\footnotetext{
DES: Replicación 6 - Compradores - - DES: Replicación 6 - Merc. Pot.

—DES: Replicación 20 - Compradores - - DES: Replicación 20 - Merc. Pot.

DES: Replicación 1 - Compradores - - DES: Replicación 1 - Merc. Pot.
}

Elaboración propia.

$\mathrm{Al}$ instante de tiempo donde se interceptan ambas curvas (del mismo color), se denomina tiempo de intersección, y determina el momento en el que la innovación ha alcanzado el 50\% del mercado potencial. Para el caso de ESR se interpreta que 100 personas ya han comprado la bicicleta y aún restan 100 más como compradores potenciales. El instante de tiempo donde ocurre esta intersección varía de una replicación a otra, justamente porque los tiempos entre adopciones siguen una distribución aleatoria. Por ejemplo, en la replicación 6 ese tiempo corresponde a un 
periodo de 9.1 quincenas, en la replicación 20 se da en un periodo de 10.4 quincenas y en la replicación 1 se da en un tiempo posterior correspondiente a 11.3 quincenas.

Por otro lado, al instante de tiempo en el que se alcanza el mayor ritmo de ventas o mayor valor de la tasa de adopción (compradores/quincena) se denomina tiempo de inflexión (o punto de inflexión), como se muestra en la Figura 3.6.

Figura 3.6.

Resultados de tres replicaciones de la Tasa de Adopción.

Tasa de Adopción

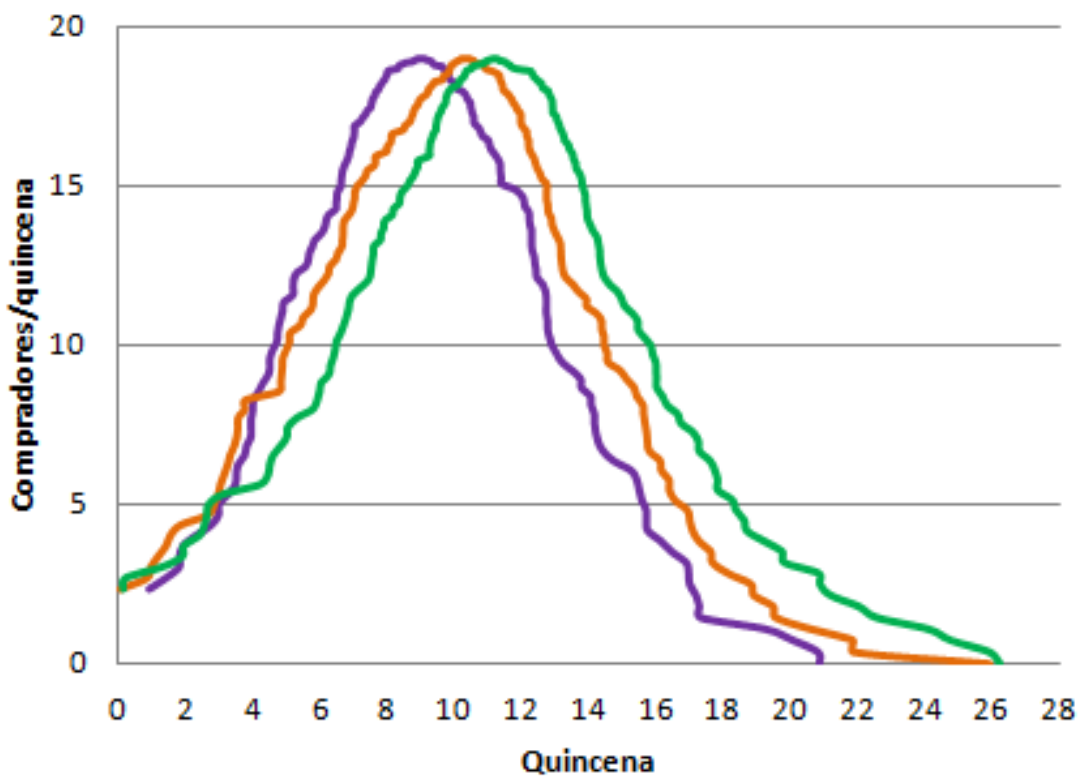

DES: Replicación 6 DES: Replicación 20 DES: Replicación 1

Elaboración propia.

En el modelo de Bass el tiempo de inflexión no puede ocurrir después del tiempo de intersección (Gómez-García, 2003). El valor de ambos tiempos está determinado por los parámetros: fracción de efectividad, tasa de contacto y fracción de adopción. En la literatura de los modelos de difusión, a la fracción de efectividad se le denomina parámetro "p" y al producto de la tasa de contacto por la fracción de adopción se le denomina parámetro "q". Mientras mayor sea la diferencia entre p y q, es decir, mientras más grande sea el término q/p entonces se cumple que el tiempo de inflexión es muy cercano al tiempo de intersección (Herrera-Madrid, 2010).

Por otro lado, la Figura 3.7 muestra el comportamiento del nivel de órdenes de producción. El incremento en este nivel viene dado por la compra de una bicicleta (que genera una nueva orden de producción) de acuerdo al modelo de difusión de Bass y la 
disminución de este nivel se da cada vez que una bicicleta empieza a ser fabricada (se inicia la producción de dicha orden y por lo tanto la orden deja la cola de espera). Al seguir los tiempos entre adopciones y de fabricación de la bicicleta distribuciones de probabilidad, entonces el nivel de las órdenes de producción también es una variable aleatoria.

Figura 3.7.

Resultados de tres replicaciones de las Ordenes de Producción.

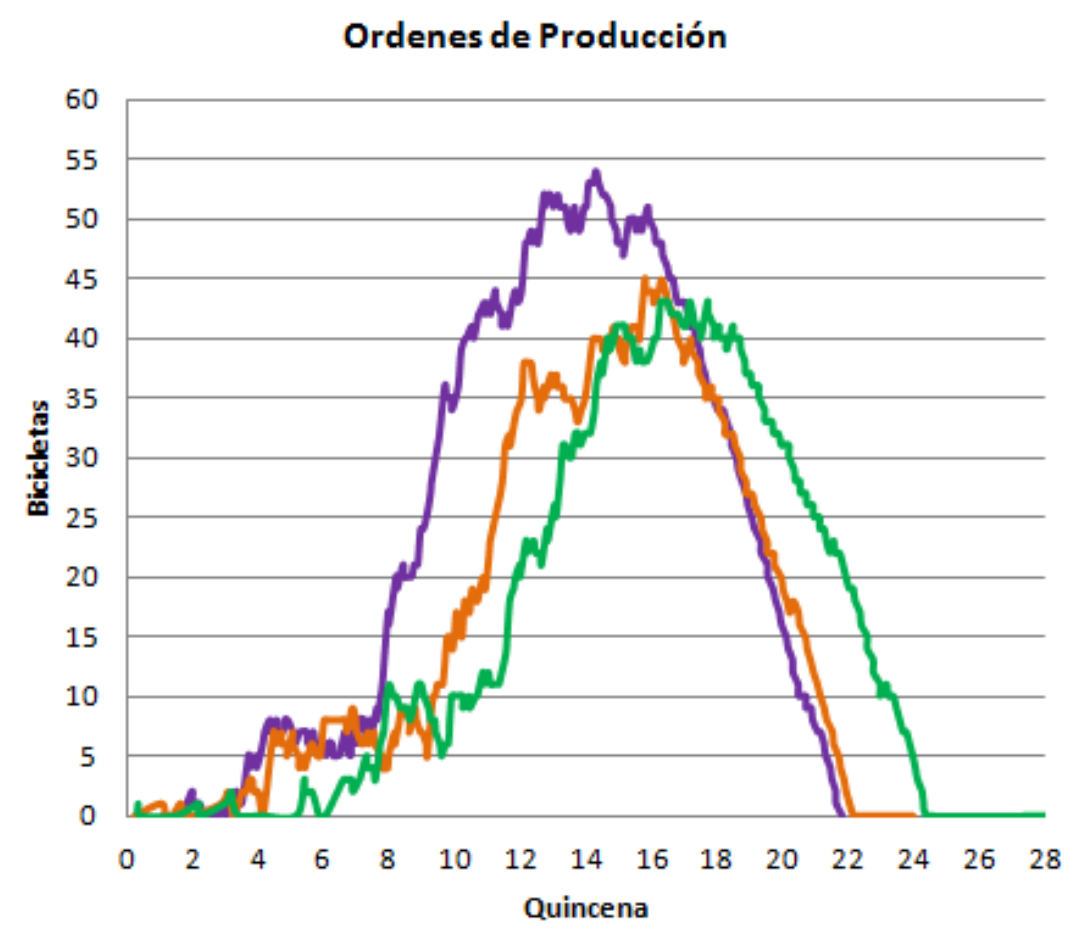

DES: Replicación 6 DES: Replicación 20 DES: Replicación 1

Elaboración propia.

\subsubsection{Valores promedio de las variables}

Una forma de recolectar la información correspondiente a "n" replicaciones es mostrando el valor promedio de las curvas asociadas con cada replicación. El tiempo total considerado en cada replicación corresponde al momento transcurrido desde la primera compra o adopción hasta que se termina de fabricar la bicicleta de la persona 200 (última adopción del mercado potencial) y ésta se encuentra en el inventario de productos terminados.

En todos los gráficos de DES donde se muestre una curva que representa al valor promedio de " $n$ " replicaciones para alguna variable o medida de 
desempeño, las coordenadas de cada punto de esa curva se representan por el par $\left(\overline{\mathrm{t}}_{\mathrm{i}}, \overline{\mathrm{s}}_{\mathrm{i}}\right)$ donde:

$\overline{\mathrm{t}}_{\mathrm{i}}$ es el tiempo promedio asociado con la entidad $\mathrm{i}$ y se representa como el promedio de los $\mathrm{t}_{\mathrm{ij}}$ que son los tiempos tomados a la entidad i en la replicación j (ver Tabla 3.1), entonces:

$\bar{t}_{\mathrm{i}}=\frac{\sum_{\mathrm{j}=1}^{\mathrm{n}} \mathrm{t}_{\mathrm{ij}}}{\mathrm{n}}$

Tabla 3.1

Valores promedio de los tiempos por replicación.

\begin{tabular}{|c|c|c|c|c|c|}
\hline Entidad & Promedio & Replicación 1 & Replicación 2 & $\ldots$ & Replicación n \\
\hline 1 & $\overline{\mathrm{t}_{1}}$ & $\mathrm{t}_{1,1}$ & $\mathrm{t}_{1,2}$ & $\ldots$ & $\mathrm{t}_{1, \mathrm{n}}$ \\
\hline 2 & $\overline{\mathrm{t}_{2}}$ & $\mathrm{t}_{2,1}$ & $\mathrm{t}_{2,2}$ & $\ldots$ & $\mathrm{t}_{2, \mathrm{n}}$ \\
\hline$:$ & $:$ & $:$ & $:$ & $:$ & $:$ \\
\hline 199 & $\overline{\mathrm{t}_{199}}$ & $\mathrm{t}_{199,1}$ & $\mathrm{t}_{199,2}$ & $\ldots$ & $\mathrm{t}_{199, \mathrm{n}}$ \\
\hline 200 & $\overline{\mathrm{t}_{200}}$ & $\mathrm{t}_{200,1}$ & $\mathrm{t}_{200,2}$ & $\ldots$ & $\mathrm{t}_{200, \mathrm{n}}$ \\
\hline
\end{tabular}

Elaboración propia.

De igual manera, $\overline{\mathrm{s}}_{\mathrm{i}}$ es el valor promedio de la variable o medida de desempeño asociado con la entidad i y se representa como el promedio de los $\mathrm{s}_{\mathrm{ij}}$ que son los valores de la variable o medida de desempeño tomados a la entidad i en la replicación j (ver Tabla 3.2), entonces:

$\overline{\mathrm{s}}_{\mathrm{i}}=\frac{\sum_{\mathrm{j}=1}^{\mathrm{n}} \mathrm{s}_{\mathrm{ij}}}{\mathrm{n}}$

Tabla 3.2.

Valores promedio de las variables por replicación.

\begin{tabular}{|c|c|c|c|c|c|}
\hline Entidad & Promedio & Replicación 1 & Replicación 2 & $\ldots$ & Replicación n \\
\hline 1 & $\overline{\mathrm{s}_{1}}$ & $\mathrm{~s}_{1,1}$ & $\mathrm{~s}_{1,2}$ & $\ldots$ & $\mathrm{s}_{1, \mathrm{n}}$ \\
\hline 2 & $\overline{\mathrm{s}_{2}}$ & $\mathrm{~s}_{2,1}$ & $\mathrm{~s}_{2,2}$ & $\ldots$ & $\mathrm{s}_{2, \mathrm{n}}$ \\
\hline$:$ & $:$ & $:$ & $:$ & $:$ & $:$ \\
\hline 199 & $\overline{\mathrm{s}_{199}}$ & $\mathrm{~s}_{199,1}$ & $\mathrm{~s}_{199,2}$ & $\ldots$ & $\mathrm{s}_{199, \mathrm{n}}$ \\
\hline 200 & $\overline{\mathrm{s}_{200}}$ & $\mathrm{~s}_{200,1}$ & $\mathrm{~s}_{200,2}$ & $\ldots$ & $\mathrm{s}_{200, \mathrm{n}}$ \\
\hline
\end{tabular}

Elaboración propia. 
Para el caso de las variables Tasa de Adopción, Compradores y Mercado Potencial, como cada entidad representa una compra del producto, entonces el tiempo $\bar{t}_{i}$ es el tiempo promedio en que ocurre la compra número i de una bicicleta.

Por ejemplo, para la variable Compradores, $\overline{\mathrm{t}}_{5}=1.93$ representa el tiempo promedio en que se realizó la compra del cliente número 5. El valor promedio de esta variables es $\overline{\mathrm{s}}_{5}=5$ compradores. En realidad $\mathrm{s}_{5 \mathrm{j}}=5$ en cada replicación j. Lo que quiere decir, que en cada replicación cuando se realiza la compra número 5 entonces la variable Compradores siempre toma un valor constante, indicando que en el instante $t_{5 j}$ hay 5 compradores. Por lo tanto, lo que va a variar en cada replicación j es el tiempo $t_{5 j}$ en el que el sistema tiene 5 compradores.

En ese mismo instante $\overline{\mathrm{t}}_{5}$, para la variable Mercado Potencial $\overline{\mathrm{s}}_{5}=195$ compradores potenciales (se resta 5 de 200). Este es un caso similar al de la variable Compradores, donde $s_{5 j}=195$ en cada replicación j y lo que varía es el tiempo $t_{5 j}$.

De forma simultánea en el instante $\overline{\mathrm{t}}_{5}$, la variable Tasa de Adopción toma el valor $\overline{\mathrm{s}}_{5}=3.705$ (que se obtiene de sumar la TA por Publicidad=195*0.01=1.95 con la TA por Boca a Boca $=5 * 8 * 195 / 200 * 0.045=1.755$ ). Otro caso similar al de las variables Compradores y Mercado Potencial, donde el valor de la variable Tasa de Adopción $\mathrm{s}_{5 \mathrm{j}}=3.705$ es el mismo en cada replicación y lo que varía es el instante de tiempo $\mathrm{t}_{5 \mathrm{j}}$ en que ocurre esa tasa.

Para el caso de la variable Ordenes de Producción y la medida de desempeño Porcentaje de Utilización de Líneas, $\overline{\mathrm{t}}_{\mathrm{i}}$ es el tiempo promedio en que se termina de fabricar la bicicleta asociada con la orden de producción número i.

Por ejemplo, para la variable Ordenes de Producción, $\overline{\mathrm{t}}_{18}=4.62$ representa el tiempo promedio en que se terminó de fabricar la orden de producción número 18. El valor promedio de esta variable es $\overline{\mathrm{s}}_{18}=2.2$ órdenes de producción en espera de ser procesadas en ese instante de tiempo. En este caso, la variable Ordenes de Producción puede tomar un valor $\mathrm{s}_{18 \mathrm{j}}$ distinto en cada replicación $\mathrm{j}$ para cada instante de tiempo $\mathrm{t}_{18 \mathrm{j}}$.

En ese mismo instante $\overline{\mathrm{t}}_{18}$, para la medida de desempeño Porcentaje de Utilización de Líneas el valor promedio de esta medida de desempeño es de $\overline{\mathrm{s}}_{18}=73 \%$. De forma similar a la variable Ordenes de Producción, esta medida de desempeño puede tomar un valor $\mathrm{s}_{18 \mathrm{j}}$ distinto en cada replicación $\mathrm{j}$ para cada instante de tiempo $\mathrm{t}_{18 \mathrm{j}}$. 
Las Figuras 3.8 y 3.9 muestran los valores promedio correspondientes a las 1000 replicaciones de las variables Compradores, Mercado Potencial, Tasa de Adopción y Ordenes de Producción.

De la Figura 3.8 se aprecia que el tiempo de intersección ocurre en la quincena 10.1. El tiempo para que todos los compradores potenciales hayan adquirido una bicicleta es aproximadamente 26 quincenas. El tiempo de inflexión ocurre en la quincena 10 (Figura 3.9). Lo cual indica que ambos puntos son bastante similares. En este caso $\mathrm{p}=0.01$ y $\mathrm{q}=0.36$ siendo $\mathrm{q} / \mathrm{p}=36$. Se verá más adelante en uno de los escenarios que un valor más pequeño de $\mathrm{q} / \mathrm{p}$ hacen que estos dos tiempos sean bien distintos.

Figura 3.8.

Valores promedio de Compradores y Mercado Potencial.

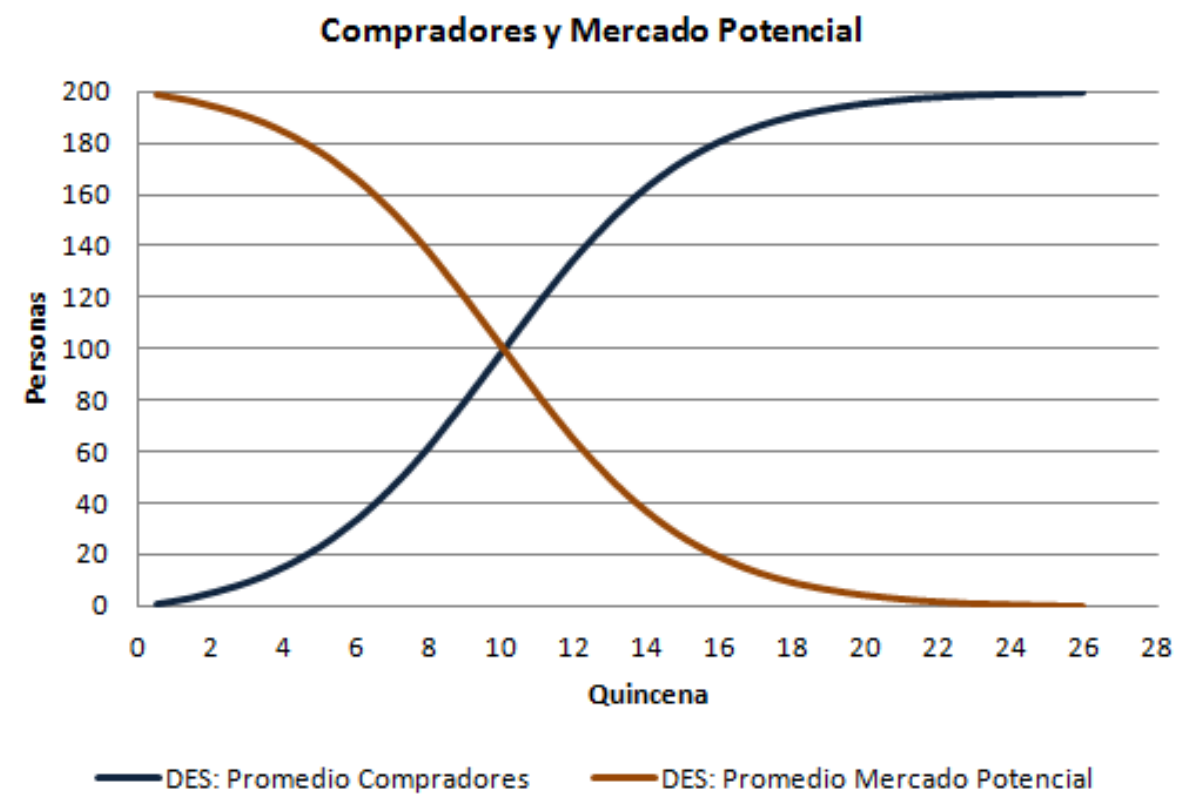

Elaboración propia.

Un aspecto interesante de resaltar es que la curva de Compradores es convexa hasta el tiempo de inflexión y luego cambia a cóncava. Además como se aprecia en la Figura 3.9, la tasa de adopción se va incrementado hasta justo antes del tiempo de inflexión y luego va disminuyendo con una tendencia a estabilizarse en cero. Esto indica que el proceso de adopción de los compradores es más acelerado al inicio hasta el tiempo de inflexión. El valor máximo de la tasa de adopción fue de 19.01 compradores por quincena.

En la Figura 3.9 también se nota que tomó en promedio 14.5 quincenas el alcanzar un nivel máximo de 54.33 órdenes de producción. Este crecimiento de las 
órdenes está relacionado con que la tasa promedio de adopción (que marca el flujo de llegada de las órdenes de producción) es mayor a la tasa máxima de producción (10 bicicletas/quincena) entre las quincenas 5.4 y 14.3. Pasada la quincena 14.3 la tasa promedio de producción supera a la de adopción y las órdenes empiezan a decrecer. Después de aproximadamente 26 quincenas ya no hay órdenes pendientes de producción.

Figura 3.9.

Valor promedio de Tasa de Adopción y Ordenes de Producción.

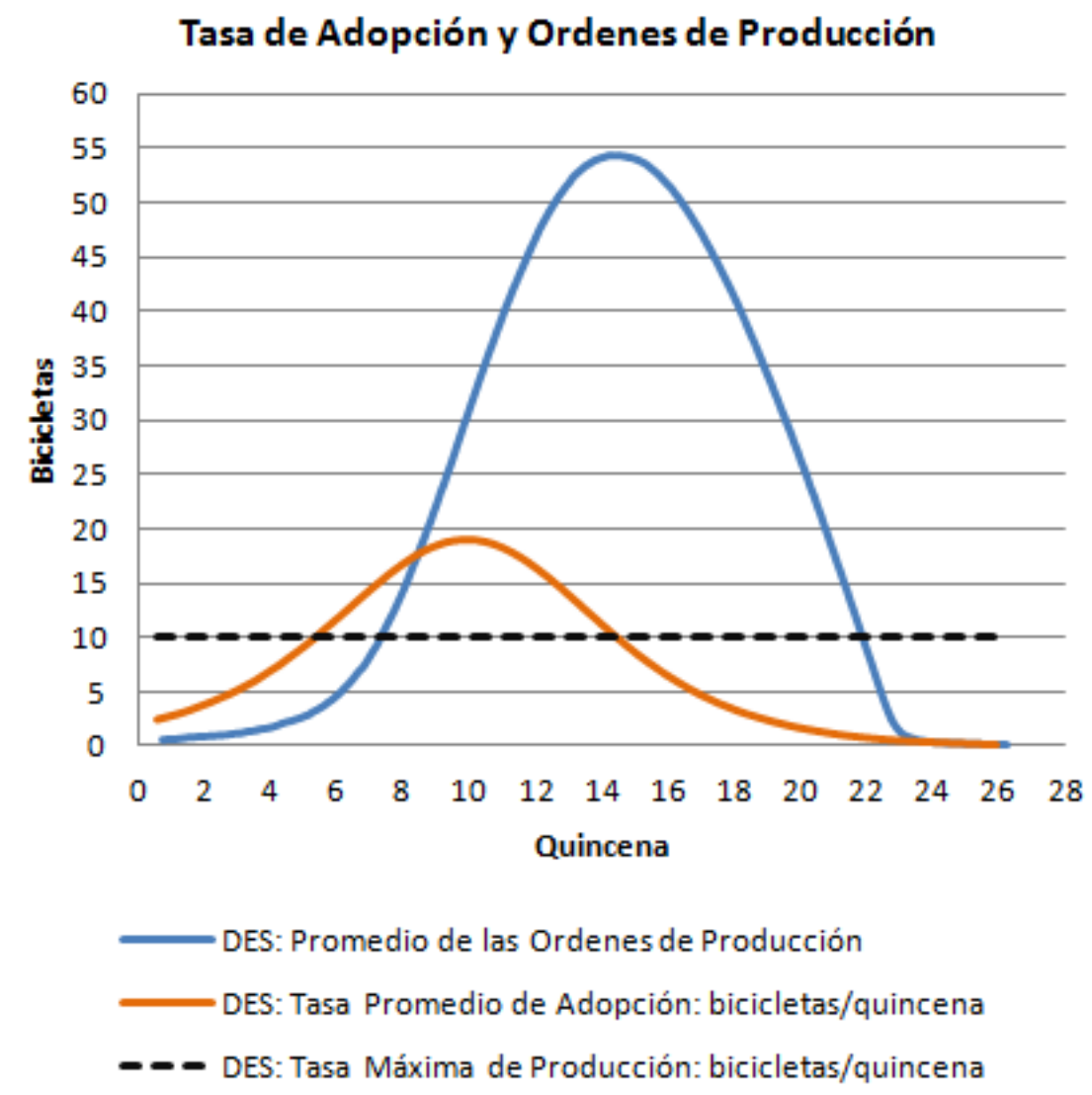

Elaboración propia.

La Figura 3.10 muestra el nivel de utilización de las dos líneas de producción.

Se trabaja a la tasa máxima de producción aproximadamente desde finales de la quincena 7 hasta mediados de la quincena 22. Trabajando a la máxima capacidad de 10 bicicletas por quincena, toma como 5.5 quincenas adicionales terminar las 54.33 órdenes de producción acumuladas hasta la quincena 14.5 (Figura 3.9). Es decir, si se cumpliera que a partir de la quincena 14.5 ya no llegaran más órdenes de producción, ESR terminaría con su órdenes pendientes en la quincena 20 (14.5+5.5). Sin embargo, 
como se muestra en la Figura 3.9, siguen llegando órdenes, aunque a un nivel decreciente y eso hace que se concluya todo el trabajo en la quincena 26.

Figura 3.10.

Valor promedio del Porcentaje de Utilización de las líneas de producción.

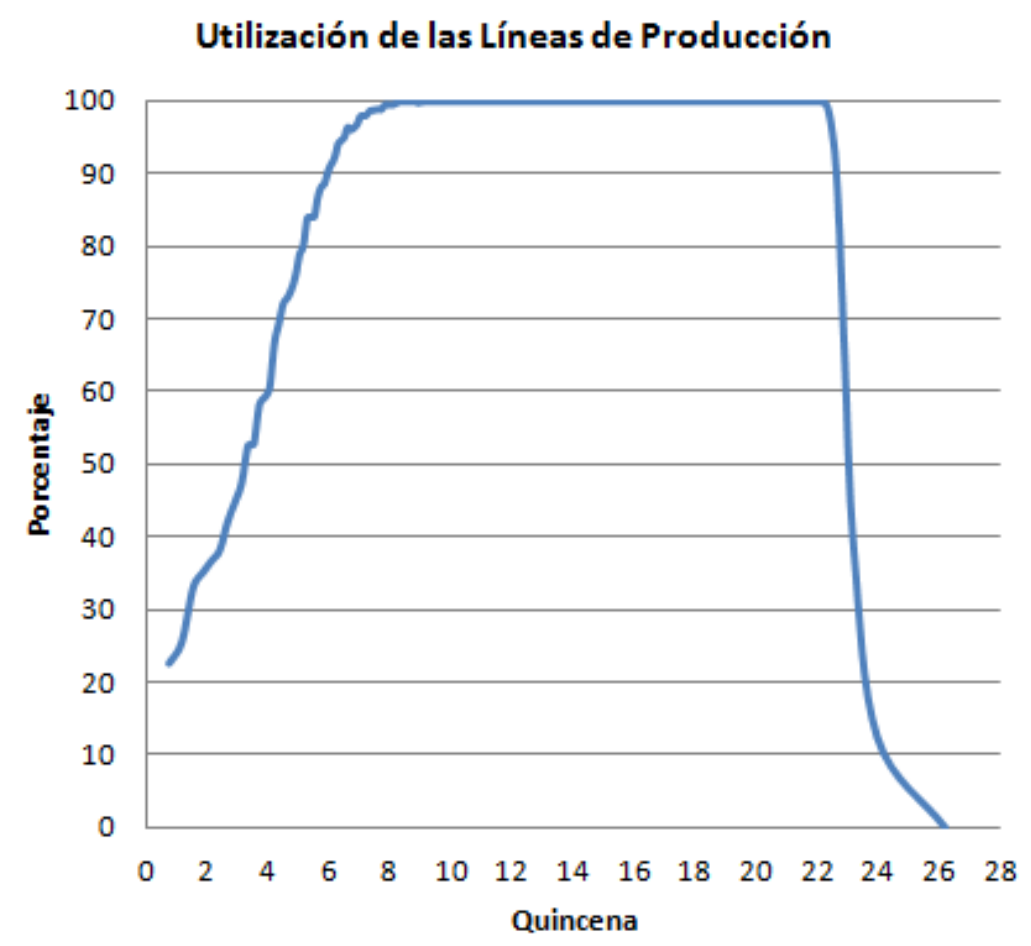

DES: Promedio de Porcentaje de Utilización

Elaboración propia.

\subsection{Modelo de dinámica de sistemas}

El modelo de SD se genera en dos etapas. Primero se elabora el diagrama causal que involucra las relaciones entre las variables y parámetros del modelo. Segundo, se procede a convertir el diagrama causal en el diagrama de Forrester que permite hacer las simulaciones requeridas.

\subsubsection{Diagrama causal del modelo}

En la Figura 3.11 se muestra el diagrama causal del modelo de negocio de ESR (pronóstico de ventas y producción de la bicicleta). Se pueden apreciar cuatro bucles, tres de ellos asociados con el proceso de adopción (B1, B2 y R3) y el último con el proceso de producción (B4). 
Analizando el proceso de adopción, el bucle B1 es un bucle de balance que me indica como se va saturando el mercado por el factor externo de la publicidad al reducirse el mercado potencial e incrementarse el número de compradores. Está conformado por las variables: Mercado Potencial, Tasa de Adopción por Publicidad y Tasa de Adopción. Este bucle tiene la siguiente interpretación: una reducción en el Mercado Potencial origina una reducción en la Tasa de Adopción por Publicidad que a su vez causa una reducción en la Tasa de Adopción. Una reducción en la Tasa de Adopción causa que el Mercado Potencial tenga una cantidad mayor de personas que si no hubiera ocurrido el cambio en la Tasa de Adopción. Esto significa que la reducción de las personas que conforman el mercado potencial no se realiza de forma abrupta sino que va convergiendo a cero.

Figura 3.11.

Diagrama causal para el modelo de negocio de ESR.

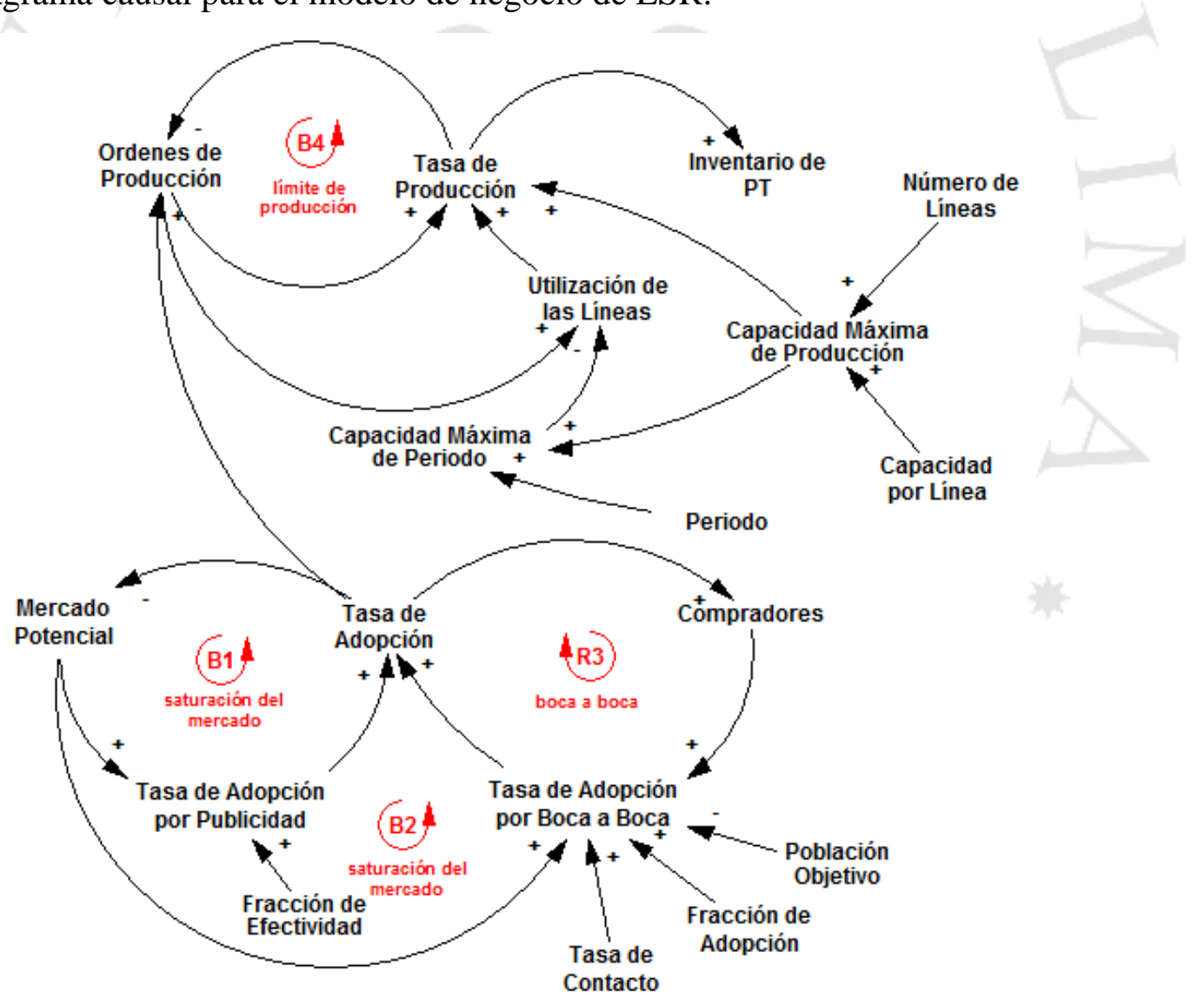

Elaboración propia.

La Tasa de Adopción por Publicidad también está influenciada por la Fracción de Efectividad, lo cual indica que mientras más efectiva es la campaña de promoción de los medios de comunicación mayor es la Tasa de Adopción. 
El bucle B2 es también un bucle de balance que indica como se va saturando el mercado por el factor interno del boca a boca. Está conformado por las variables: Mercado Potencial, Tasa de Adopción por Boca a Boca y Tasa de Adopción. Este bucle tiene la siguiente interpretación: una reducción en el Mercado Potencial origina una reducción en la Tasa de Adopción por Boca a Boca que a su vez causa una reducción en la Tasa de Adopción. Una reducción en la Tasa de Adopción causa que el Mercado Potencial tenga una cantidad mayor de personas que si no hubiera ocurrido el cambio en la Tasa de Adopción.

La Tasa de Adopción por el Boca a Boca está influenciada positivamente por la Tasa de Contacto y la Fracción de Adopción, lo cual indica que ambos: un mayor contacto entre las personas (mayor tendencia a socializar) o un amento en la Fracción de Adopción (mayor impacto de la innovación) implican un aumento en la Tasa de Adopción. Por el contrario, la Población Objetivo tiene una influencia negativa en la Tasa de Adopción. Al incrementarse la Población Objetivo entonces la probabilidad de un contacto favorable se reduce implicando una disminución en la Tasa de Adopción.

El bucle R3 es un bucle de refuerzo que me indica como se incrementa el número de compradores. Está conformado por las variables: Compradores, Tasa de Adopción por Boca a Boca y Tasa de Adopción. Este bucle tiene la siguiente interpretación: un aumento en el número de Compradores origina un incremento en la Tasa de Adopción por el Boca a Boca, la cual a su vez causa un aumento en la Tasa de Adopción. Un incremento de la Tasa de Adopción causa que el número de Compradores aumente en una cantidad mayor que si no hubiera ocurrido el cambio en la Tasa de Adopción.

Analizando el proceso de producción, el bucle B4 es un bucle de balance que me indica como se van fabricando las órdenes de producción. Este bucle tiene la siguiente interpretación: un aumento en las Ordenes de Producción origina un incremento en la Tasa de Producción. Un aumento en la Tasa de Producción causa que el número de Ordenes de Producción disminuya en una cantidad mayor que si no hubiera ocurrido el cambio en la Tasa de Producción.

La Tasa de Producción está influenciada positivamente por la Utilización de la Capacidad y la Capacidad Máxima de Producción. De igual manera, la capacidad 
Máxima de Producción está influenciada positivamente por el Número de Líneas (de producción) y la Capacidad por Línea.

La Capacidad Máxima de Periodo indica cual es el máximo nivel de producción que se puede conseguir para un periodo de análisis generalmente distinto al definido en la Capacidad Máxima de Producción. Por ejemplo, si la Capacidad Máxima de Producción fue definida como 10 bicicletas por quincena entonces la máxima producción que se logra en el periodo de una semana es 5 bicicletas. El periodo utilizado es el definido por el $\Delta \mathrm{t}$ ó TIME STEP en el modelo de SD. Finalmente, la Capacidad Máxima de Periodo está influenciada positivamente por ambos: el Periodo y la Capacidad Máxima de Producción.

La Utilización de las Líneas está influenciada positivamente por las Ordenes de Producción. Si éstas últimas se incrementan entonces hay una mayor Utilización de las Líneas. Por el contrario, si la Capacidad Máxima de Periodo se incrementa esto reduce la Utilización de las Líneas, al tener el mismo número de órdenes de producción pero en una capacidad de producción mayor.

Se mencionaron en los párrafos anteriores la interpretación de las variables y sus interconexiones con los bucles del modelo. Sin embargo, hay que hacer notar que existen dos bucles más de balance que denominaremos B5 y B6 que no son fáciles de distinguir a primera vista. Mientras que los bucles B1 y B2 afectan exclusivamente a la variable Mercado Potencial y el bucle R3 afecta exclusivamente a la variable Compradores. Los bucles B5 y B6 afectan a ambas variables simultáneamente. Si aislamos la parte del diagrama causal que corresponde únicamente al pronóstico de la demanda, entonces en la Figura 3.12 se muestra el bucle B5 conformado por las variables: Mercado Potencial, Tasa de Adopción por Publicidad, Tasa de Adopción (que suma a Compradores), Compradores, Tasa de Adopción por Boca a Boca y Tasa de adopción (que resta a Mercado Potencial). Este bucle tiene la siguiente interpretación: una reducción en el Mercado Potencial origina una reducción en la Tasa de Adopción por Publicidad que a su vez causa una reducción en la Tasa de Adopción (que afecta a los compradores). Una reducción en la Tasa de Adopción origina que disminuya el número de Compradores y esto genera una reducción en la Tasa de Adopción por Boca a Boca que a su vez causa una reducción en la Tasa de Adopción (que afecta al mercado potencial). Una reducción en la Tasa de Adopción causa que el 
Mercado Potencial tenga una cantidad mayor de personas que si no hubiera ocurrido el cambio en la Tasa de Adopción.

Figura 3.12.

Diagrama causal para el bucle de balance B5.

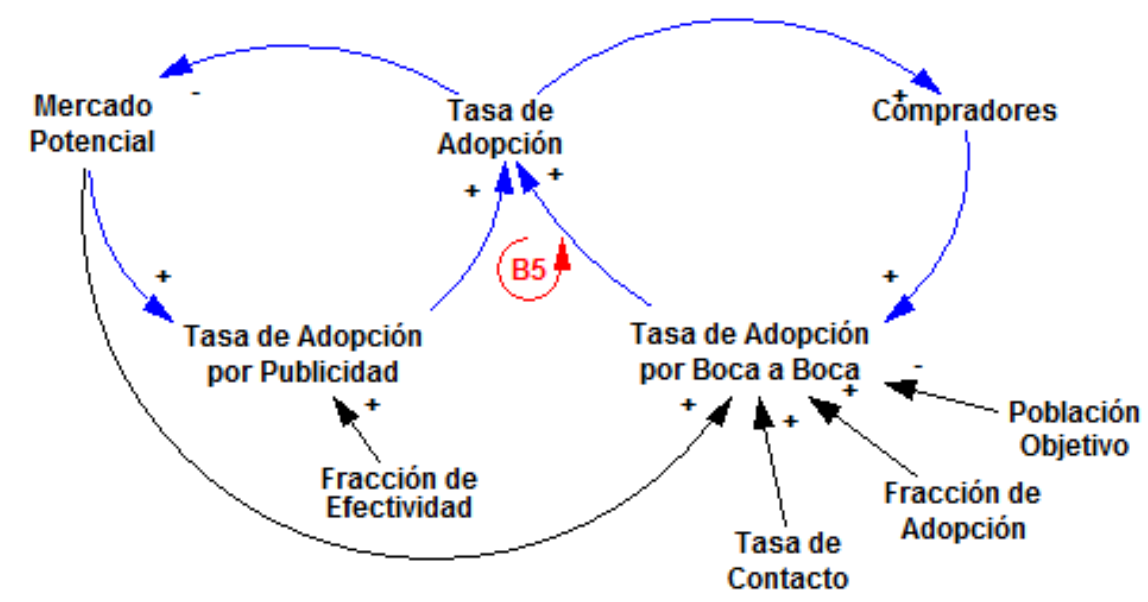

Elaboración propia.

En la Figura 3.13 se muestra el bucle B6. Está conformado por las variables: Mercado Potencial, Tasa de Adopción por Boca a Boca, Tasa de Adopción (que suma a Compradores), Compradores, Tasa de Adopción por Boca a Boca y Tasa de adopción (que resta a Mercado Potencial).

Figura 3.13.

Diagrama causal para el bucle de balance B6.

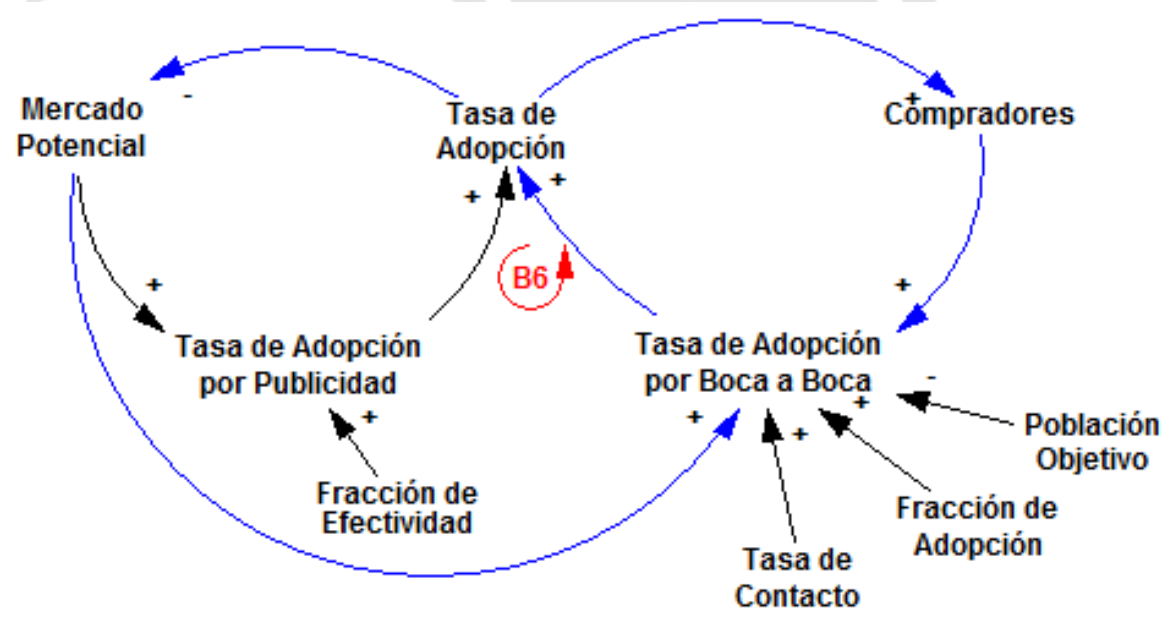

Elaboración propia.

Este bucle tiene la siguiente interpretación: una reducción en el Mercado Potencial origina una reducción en la Tasa de Adopción por Boca a Boca que a su vez causa una reducción en la Tasa de Adopción (que afecta a los compradores). Una 
reducción en la Tasa de Adopción origina que disminuya el número de Compradores y esto genera una reducción en la Tasa de Adopción por Boca a Boca que a su vez causa una reducción en la Tasa de Adopción (que afecta al mercado potencial). Una reducción en la Tasa de Adopción causa que el Mercado Potencial tenga una cantidad mayor de personas que si no hubiera ocurrido el cambio en la Tasa de Adopción.

\subsubsection{Diagrama de Forrester del modelo}

Hecho el análisis de causalidad entre las variables del modelo se procede a la conversión del diagrama causal al diagrama de Forrester, como se muestra en la Figura 3.14.

Figura 3.14.

Diagrama de Forrester para el modelo de negocio de ESR.

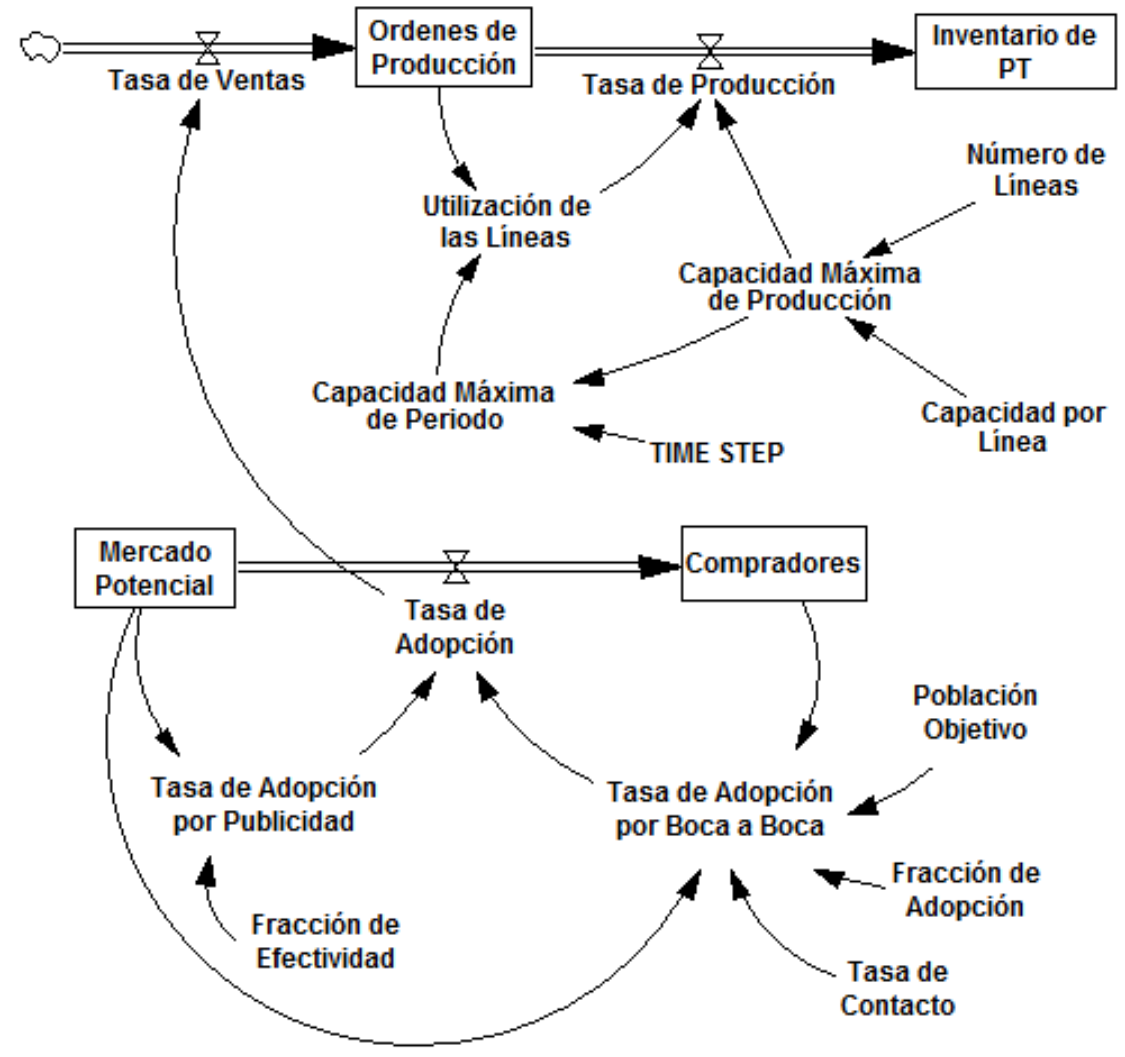

Elaboración propia.

Esto se logra haciendo que las tasas sean variables de flujo. Además se definen cuatro variables de nivel: Mercado Potencial, Compradores, Ordenes de Producción e Inventario de Productos Terminados. Este diagrama se ha creado usando el software VENSIM (Ventana Systems, 2013). El código completo del modelo se puede ver en el Anexo 2. 
En la Figura 3.14, la Tasa de Adopción es igual a la Tasa de Ventas. La primera influye en la variable Compradores y la segunda en la variable Ordenes de Producción. Aunque se sabe que cada comprador genera una orden de producción, se debió usar dos variables distintas: una para los compradores y otra para las órdenes de producción. Eso debido a que, por un lado, se debe mantener un número acumulado de los compradores (necesario para el cálculo de la Tasa de Adopción por Boca a Boca) mientras que por otro lado las órdenes de producción van a ir disminuyendo conforme se vayan fabricando las bicicletas.

La Figura 3.15 muestra las curvas de las variables Compradores y Mercado Potencial generadas por el modelo de SD.

Figura 3.15.

Comparación SD y DES: Compradores y Mercado Potencial.

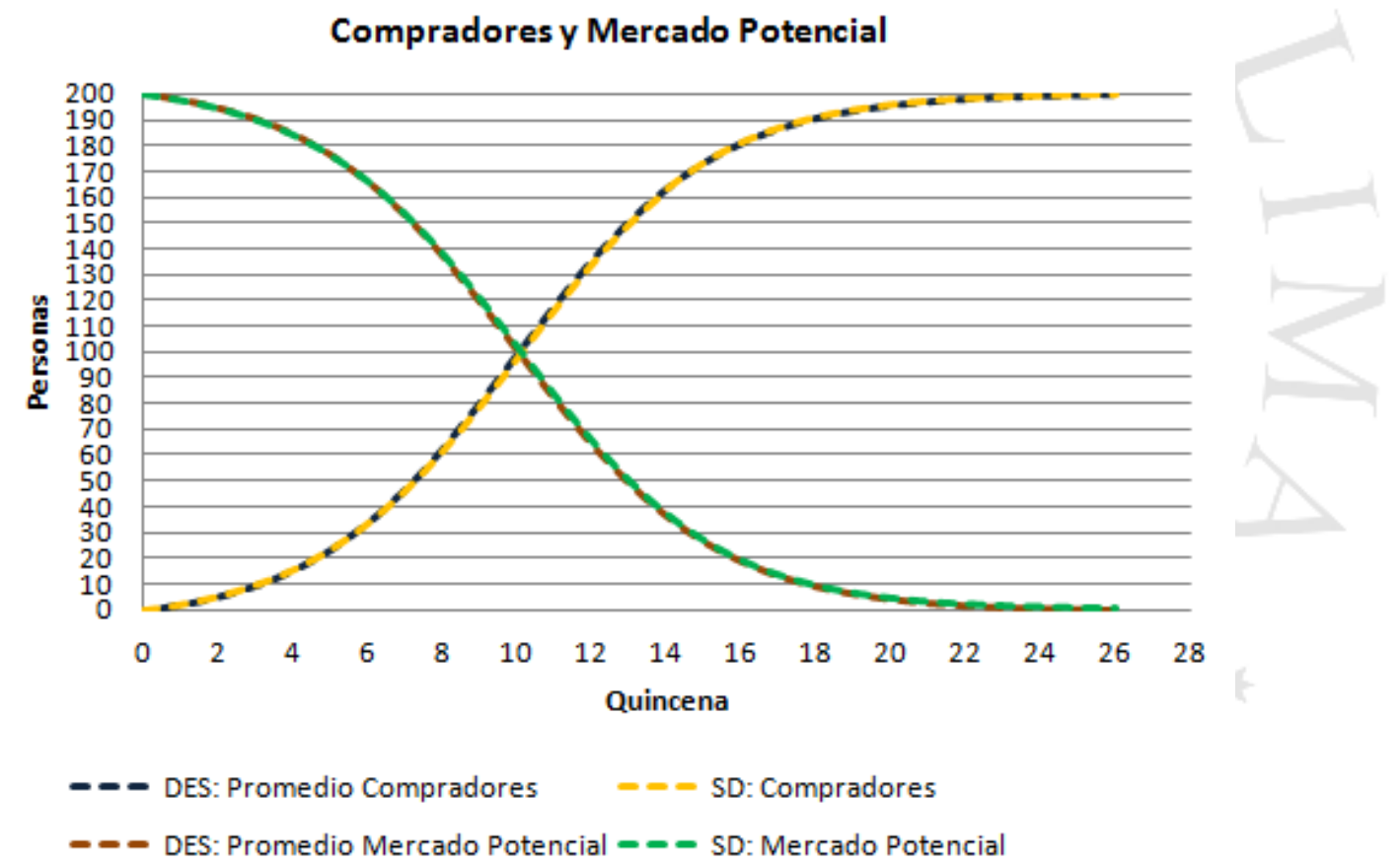

Elaboración propia.

Mientras que la Figura 3.16 muestra la curva de la Tasa de Adopción. Al trabajar el modelo de $\mathrm{SD}^{6}$ con valores determinísticos, sólo se requiere un corrida de la simulación. Además estas curvas se comparan con las curvas promedio generadas por el modelo de DES y se aprecia que son equivalentes.

\footnotetext{
$6 \quad$ El valor de $\Delta t$ usado en los modelos de SD en este documento es de 0.25 a menos que se indique lo contrario.
} 
Figura 3.16.

Comparación SD y DES: Tasa de Adopción.

Tasa de Adopción

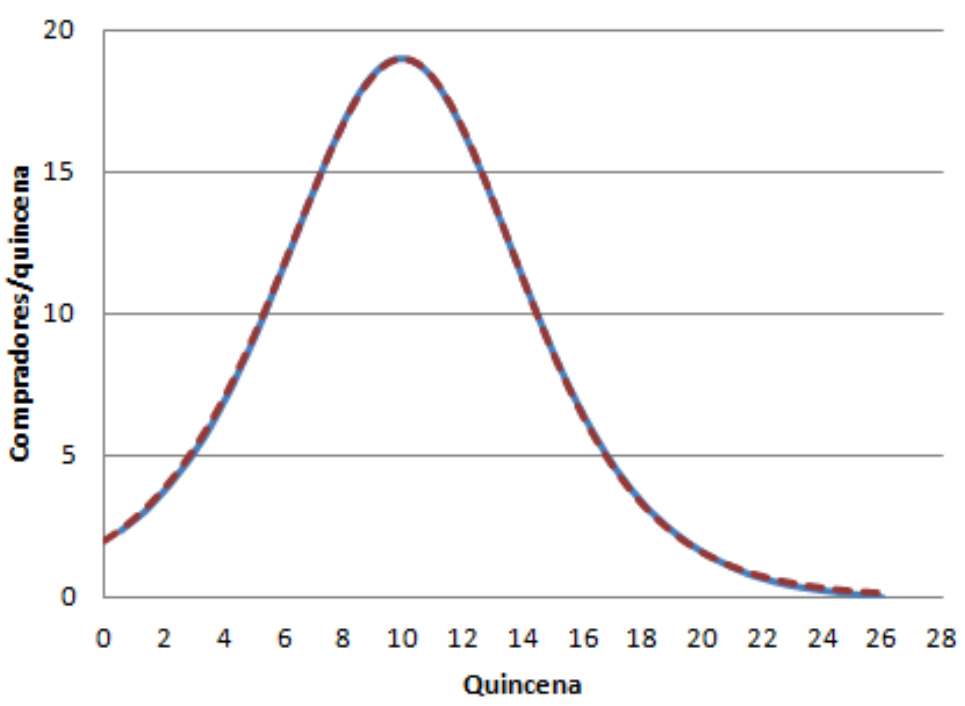

DES: Promedio - - - SD

Elaboración propia.

La Figura 3.17 muestra el comportamiento de la variable Ordenes de Producción.

Figura 3.17.

Comparación SD y DES: Ordenes de Producción.

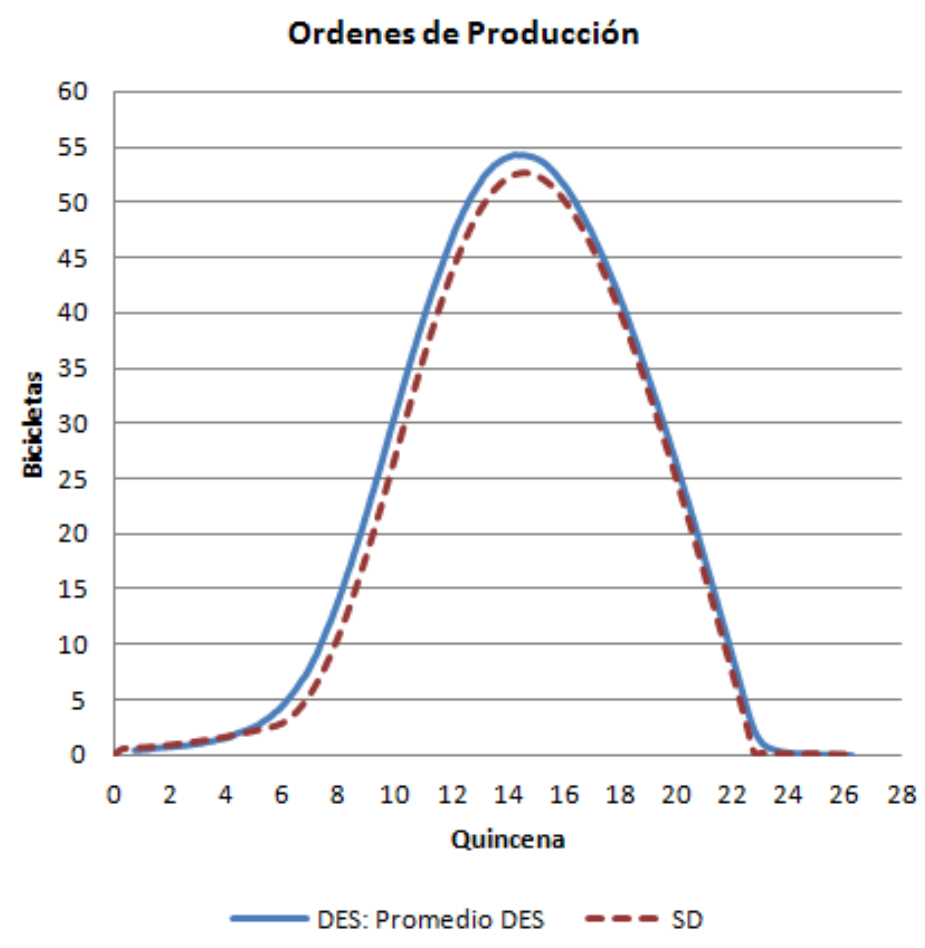

Elaboración propia. 
La Figura 3.18 muestra la medida de desempeño Porcentaje de Utilización de las Líneas generado por el modelo de SD. Se aprecia que en ambos casos las curvas también son bastante similares a las generadas con el modelo de DES.

Hay que recordar que en el caso de las variables Compradores, Mercado Potencial y Tasa de Adopción la variabilidad asociada viene originada por la aleatoriedad de los tiempos entre adopciones. Por otro lado, en el caso de las Ordenes de Producción, esta variabilidad es mayor, al estar afectada no solo por el tiempo entre adopciones, sino que además se ve afectada por el tiempo de fabricación. Y esa mayor variación se ve reflejada también en la utilización de las líneas de producción.

Figura 3.18.

Comparación SD y DES: Utilización de las Líneas.

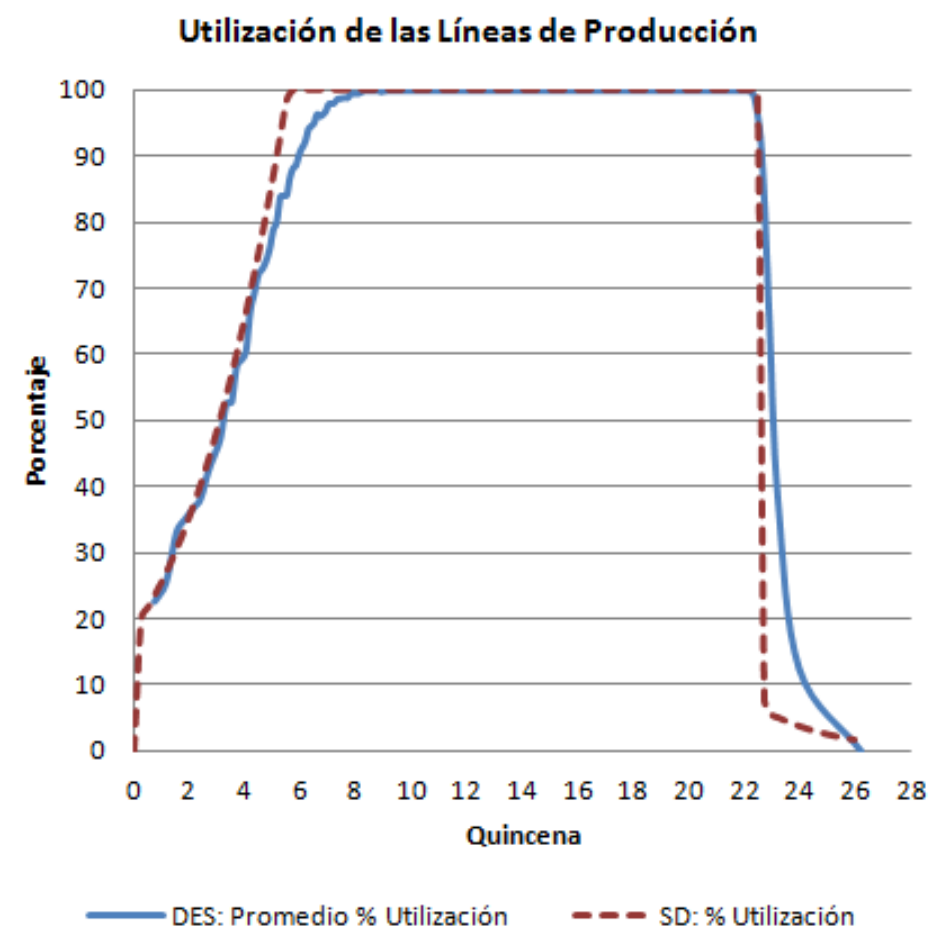

Elaboración propia.

\subsection{Modelo de simulación basada en agentes}

En el caso de ABS también se trabaja una versión estocástica del modelo de Bass. Sin embargo, a diferencia del modelo usado para DES en la sección 3.4, la versión de ABS no requiere definir una ecuación para el cálculo de la tasa de adopción. Es decir, no existe una tasa de adopción predefinida, sino que el número de compradores del producto resulta de la interacción entre un comprador potencial y un comprador. 
En el caso de SD el propósito de usar la tasa de adopción es el de determinar el número agregado de compradores de un periodo de tiempo a otro. El caso de DES es similar. Aunque cada entidad representa a una persona que ya ha adoptado el producto, se usa el tiempo entre adopciones (compras) sucesivas para representar el resultado de dichas interacciones (como un lapso de tiempo transcurrido) pero no se representan cada una de las interacciones como tal.

Como ABS trabaja a nivel de interacciones entre individuos, lo que se reproduce en ese modelo son cada una de esas interacciones y luego se agregan esos resultados para determinar el número de compradores. Es debido a ello que ABS requiere mucho más esfuerzo computacional que SD ó DES. La ventaja es que se gana flexibilidad para poder mostrar el comportamiento de sistemas en situaciones donde las interrelaciones entre los individuos no son fáciles de representar en una ecuación matemática. Un ejemplo de esta dificultad para el cálculo de la tasa de adopción se podrá apreciar más adelante en las secciones 3.7.5 y 3.7.6.

Se usará el software NETLOGO (Wilensky, 1999) de ABS para construir el modelo de negocio de ESR. Como NETLOGO no tiene un ambiente gráfico de modelamiento similar a VENSIM o ARENA, se mostrará en la Figura 3.19 un diagrama de bloques con los pasos más relevantes de la lógica del modelo. El código completo del modelo se puede ver en el Anexo 3. A diferencia de ARENA donde, por ejemplo, es muy fácil cambiar las prioridades de entidades en las colas mediante el uso de selección de opciones en menús, en NETLOGO cualquiera de estos procesos se deben programar completamente.

El avance del reloj de simulación se realiza de forma similar a como se hace en $\mathrm{SD}$, es decir, en intervalos constantes de tiempo. El número de iteraciones que se requieren en una replicación del modelo se calcula de la siguiente forma:

Número de iteraciones por quincena $=\frac{200 \text { personas } x \frac{8 \text { personas }}{\text { persona } x \text { quincena }}}{2 \frac{\text { personas }}{\text { iteración }}}=800 \frac{\text { iteraciones }}{\text { quincena }}$

El número de 8 personas que se contactan por quincena, por cada persona de la población objetivo, viene dado de la tasa de contacto definida en la sección 3.3. Por otro lado, en cada iteración del boca a boca hay 2 personas involucradas. Considerando los resultados de los modelos de DES y SD que muestran un horizonte de tiempo promedio de 26 quincenas para fabricar todas la órdenes de producción entonces tenemos: 
Número de iteraciones (por replicación) $=$

$800 \frac{\text { iteraciones }}{\text { quincena }} \times 26$ quincenas $=20800$ iteraciones

Figura 3.19.

Diagrama de bloques con la lógica del modelo de ABS.

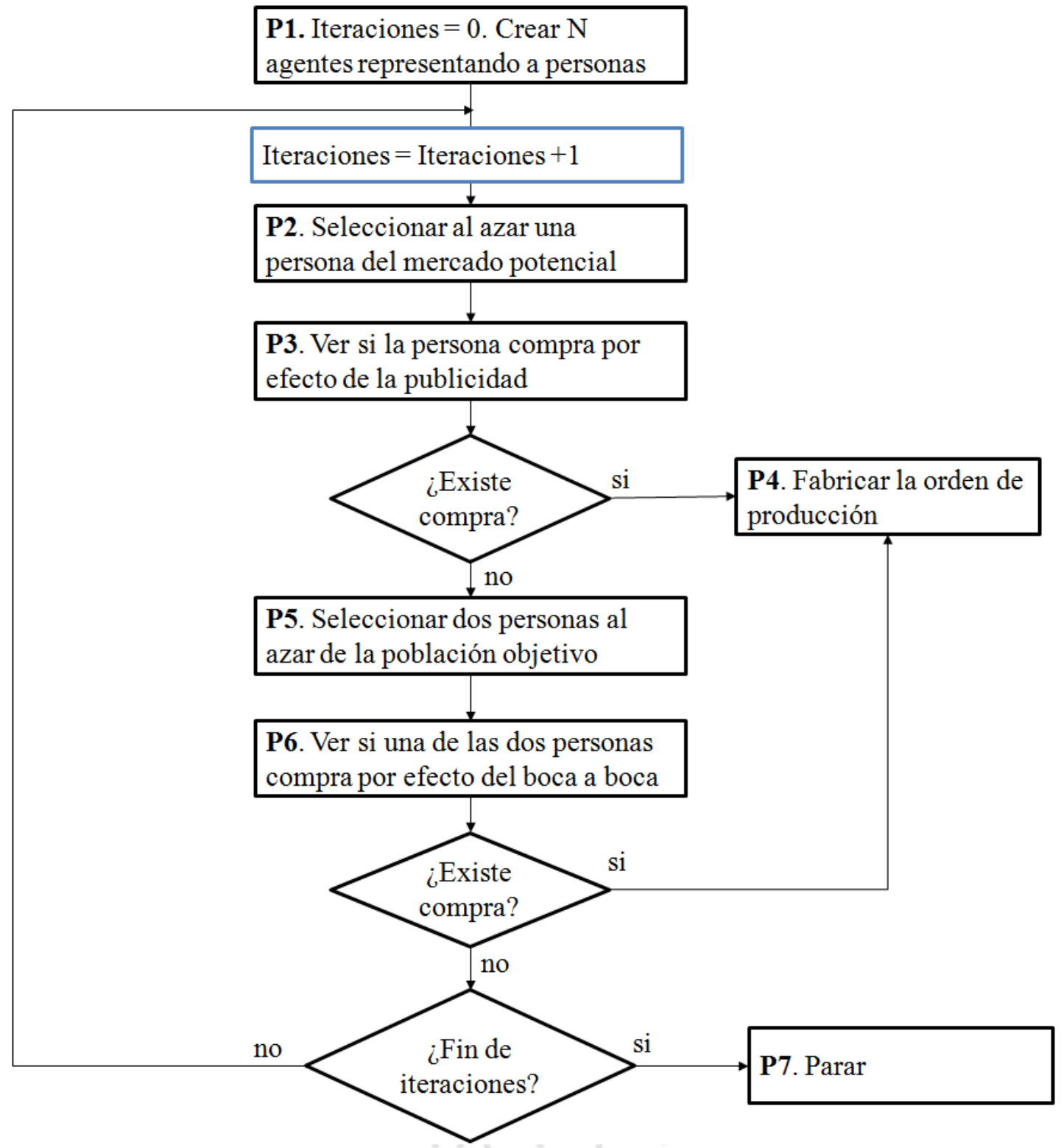

Elaboración propia.

A continuación se hace una explicación más detallada de cada uno de los bloques de la Figura 3.19.

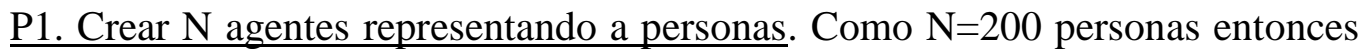
se crean 200 agentes del tipo personas. Se coloca para cada agente dos atributos: uno binario que guarda la información si ha adoptado el producto (valor 1) o no ha adoptado 
(valor 0). El otro atributo guarda la probabilidad de adopción por el boca a boca que en este caso es constante e igual a 0.045 .

$\underline{\mathrm{P} 2 . \text { Seleccionar al azar una persona del mercado potencial. El mercado potencial }}$ está conformado por todas las personas cuyo atributo de haber adoptado es igual a cero. NETLOGO tiene una función que selecciona una de esas personas al azar.

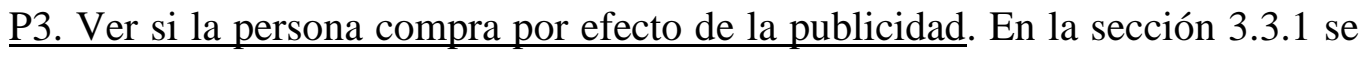
definió la tasa de adopción por publicidad como el porcentaje de los compradores potenciales que adquieren el producto en un periodo de tiempo dado (que es el $1 \%$ quincenal). Este es un porcentaje agregado sobre el mercado potencial, el cual se debe transformar en una probabilidad de compra que afecte a un sólo individuo. En la tercera columna de la Tabla 3.3 se muestran el número de compradores por quincena debido únicamente al efecto de la publicidad. Por otro lado, se sabe que en cada iteración una persona se ve influenciada por la publicidad entonces si dividimos el número de compradores por quincena entre las 800 iteraciones que se dan en cada quincena obtenemos la probabilidad de compra. Por ejemplo, se sabe que al final de la primera quincena dos personas deben haber adquirido el producto entonces como hay 800 iteraciones en cada quincena, dos de esas iteraciones deben llegar a una compra. La probabilidad sería $2 / 800=0.0025$.

Tabla 3.3.

Probabilidades de compra por efecto de la publicidad.

\begin{tabular}{cccc}
\hline Quincena & Merc. Potencial & Compradores & Probabilidad \\
\hline 1 & 200.0 & 2.00 & 0.0025 \\
2 & 198.0 & 1.98 & 0.0025 \\
3 & 196.0 & 1.96 & 0.0025 \\
4 & 194.1 & 1.94 & 0.0024 \\
5 & 192.1 & 1.92 & 0.0024 \\
6 & 190.2 & 1.90 & 0.0024 \\
7 & 188.3 & 1.88 & 0.0024 \\
8 & 186.4 & 1.86 & 0.0023 \\
9 & 184.5 & 1.85 & 0.0023 \\
10 & 182.7 & 1.83 & 0.0023 \\
11 & 180.9 & 1.81 & 0.0023 \\
12 & 179.1 & 1.79 & 0.0022 \\
13 & 177.3 & 1.77 & 0.0022 \\
14 & 175.5 & 1.76 & 0.0022 \\
15 & 173.7 & 1.74 & 0.0022 \\
16 & 172.0 & 1.72 & 0.0022
\end{tabular}




\begin{tabular}{cccc}
\hline Quincena & Merc. Potencial & Compradores & Probabilidad \\
\hline 17 & 170.3 & 1.70 & 0.0021 \\
18 & 168.6 & 1.69 & 0.0021 \\
19 & 166.9 & 1.67 & 0.0021 \\
20 & 165.2 & 1.65 & 0.0021 \\
21 & 163.6 & 1.64 & 0.0020 \\
22 & 161.9 & 1.62 & 0.0020 \\
23 & 160.3 & 1.60 & 0.0020 \\
24 & 158.7 & 1.59 & 0.0020 \\
25 & 157.1 & 1.57 & 0.0020 \\
26 & 155.6 & 1.56 & 0.0019 \\
\hline
\end{tabular}

Elaboración propia.

Se puede apreciar que se inicia con una probabilidad de 0.0025 en la primera quincena y aproximadamente cada cuatro quincenas esa probabilidad disminuye en 0.0001 .

$\underline{\text { P4. Fabricar la orden de producción. Ese proceso es similar al que se tiene en }}$ DES, cada vez que hay una compra se genera una orden de producción. Estas órdenes se envían a las líneas de producción para su fabricación, y si ambas líneas están ocupadas entonces se forma una cola de espera. Una vez procesada la orden de producción deja el sistema rumbo al almacén de productos terminados.

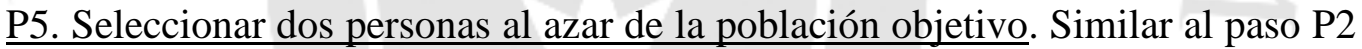
pero en este caso se seleccionan dos personas de la población objetivo, es decir, aquí si se pueden dar tres posibilidades: (1) que ambas personas ya hayan comprado, (2) que ninguna de las dos personas haya comprado el producto y (3) que sólo una de las dos personas haya comprado el producto. Es este último caso el que puede generar una adopción por el efecto boca a boca.

P6. Ver si una de las dos personas compra por el efecto del boca a boca. Para la persona que no ha adoptado el producto se toma su probabilidad de adopción por boca a boca y se compara dicho valor con el de un número aleatorio entre 0 y 1 . Si sale un valor del número aleatorio menor a dicha probabilidad entonces la persona compra el producto.

P7. Parar. Cuando se llega al número de 20,800 iteraciones se detiene el programa y se graban en un archivo las variables objeto de estudio.

Al correr el modelo de ABS en cada iteración puede suceder que dos personas como máximo compren el producto. La primera persona podría adoptar el producto por 
el efecto de la publicidad y la segunda persona podría adoptar el producto por el efecto del boca a boca. Ambos efectos son independientes uno del otro, por lo tanto, la probabilidad de que dos personas adopten el producto en una misma iteración es muy baja. Un análisis similar en el modelo de DES para que dos personas compren el producto de forma simultánea, es necesario que el tiempo entre adopciones sea prácticamente cero y eso es muy poco probable con los datos de este caso de estudio.

Como cada quincena representa 800 iteraciones entonces cada iteración es equivalente a 1/800 quincenas. En la Figura 3.20 se muestra (a modo de ejemplo) que el modelo de ABS calcula todas las iteraciones entre las personas hasta que se realiza una compra. Mientras que en modelo de DES sólo se consideran las compras ya realizadas. Esto genera una diferencia computacional de ambos paradigmas, ya que mientras que en el modelo de DES en cada replicación solo se generan 200 entidades correspondientes a las 200 personas del mercado objetivo, en el modelo de ABS en cada replicación se deben calcular 20800 iteraciones.

Figura 3.20.

Cronograma de iteraciones en ABS.

\section{Modelo de Agentes}

\begin{tabular}{|c|c|c|c|}
\hline Iteración & Estado del agente & Quincena & \\
\hline Iteración 1 & No ha adoptado & $1 / 800$ & No se ha comprado en las primeras \\
\hline $\begin{array}{c}: \\
\text { Iteración } 49\end{array}$ & $\begin{array}{c}: \\
\text { No ha adoptado }\end{array}$ & $\begin{array}{c}: \\
49 / 800\end{array}$ & 49 iteraciones \\
\hline Iteración 50 & SI ha adoptado & $50 / 800$ & $\longrightarrow$ Coincide con la 1ra compra en DES \\
\hline $\begin{array}{l}\text { Iteración } 51 \\
\quad:\end{array}$ & $\begin{array}{c}\text { No ha adoptado } \\
\text { : }\end{array}$ & $\begin{array}{l}51 / 800 \\
:\end{array}$ & No se ha comprado en las siguientes \\
\hline Iteración 352 & No ha adoptado & $352 / 800$ & 302 iteraciones \\
\hline Iteración 353 & SI ha adoptado & $353 / 800$ & $\longrightarrow$ Coincide con la 2 da compra en DES \\
\hline
\end{tabular}

Elaboración propia.

Es debido a ese mayor esfuerzo computacional que requiere ABS, que sólo se corrieron 100 replicaciones, a diferencia de las 1000 replicaciones que se corrieron con el modelo de DES. De todas formas estas 100 replicaciones implicaron 2,080,000 iteraciones.

Similar al caso de DES, para las variables de estudio, se construyen las curvas que representa al valor promedio de "n" replicaciones. Las coordenadas de cada punto de esas curvas se representan por el par $\left(\bar{t}_{\mathrm{k}}, \overline{\mathrm{s}}_{\mathrm{i}}\right)$ donde: 
$\overline{\mathrm{t}}_{\mathrm{k}}$ es el tiempo promedio asociado con la iteración $\mathrm{k}$ y se representa como el promedio de los $t_{k j}$ que son los tiempos tomados en la iteración $k$ en la replicación j, entonces:

$\overline{\mathrm{t}}_{\mathrm{k}}=\frac{\sum_{\mathrm{j}=1}^{\mathrm{n}} \mathrm{t}_{\mathrm{kj}}}{\mathrm{n}}$

De igual manera, $\overline{\mathrm{s}}_{\mathrm{k}}$ es el valor promedio de la variable o medida de desempeño asociado con la iteración $\mathrm{k}$ y se representa como el promedio de los $\mathrm{s}_{\mathrm{kj}}$ que son los valores de la variable o medida de desempeño tomados en la iteración $\mathrm{k}$ en la replicación j, entonces: $\overline{\mathrm{s}}_{\mathrm{k}}=\frac{\sum_{\mathrm{j}=1}^{\mathrm{n}} \mathrm{s}_{\mathrm{kj}}}{\mathrm{n}}$

La Figura 3.21 muestra las curvas de las variables Compradores y Mercado Potencial generadas por el modelo de ABS. Además estas curvas se comparan con las curvas promedio generadas por el modelo de DES y se aprecia que son equivalentes. Como se mencionó anteriormente, el modelo de ABS no requiere de una tasa de adopción para determinar el número de compradores. Por lo tanto, no se generó dicha curva a diferencia de los modelos de SD y DES que si la requieren.

Figura 3.21.

Comparación ABS y DES: Compradores y Mercado Potencial.

Compradores y Mercado Potencial
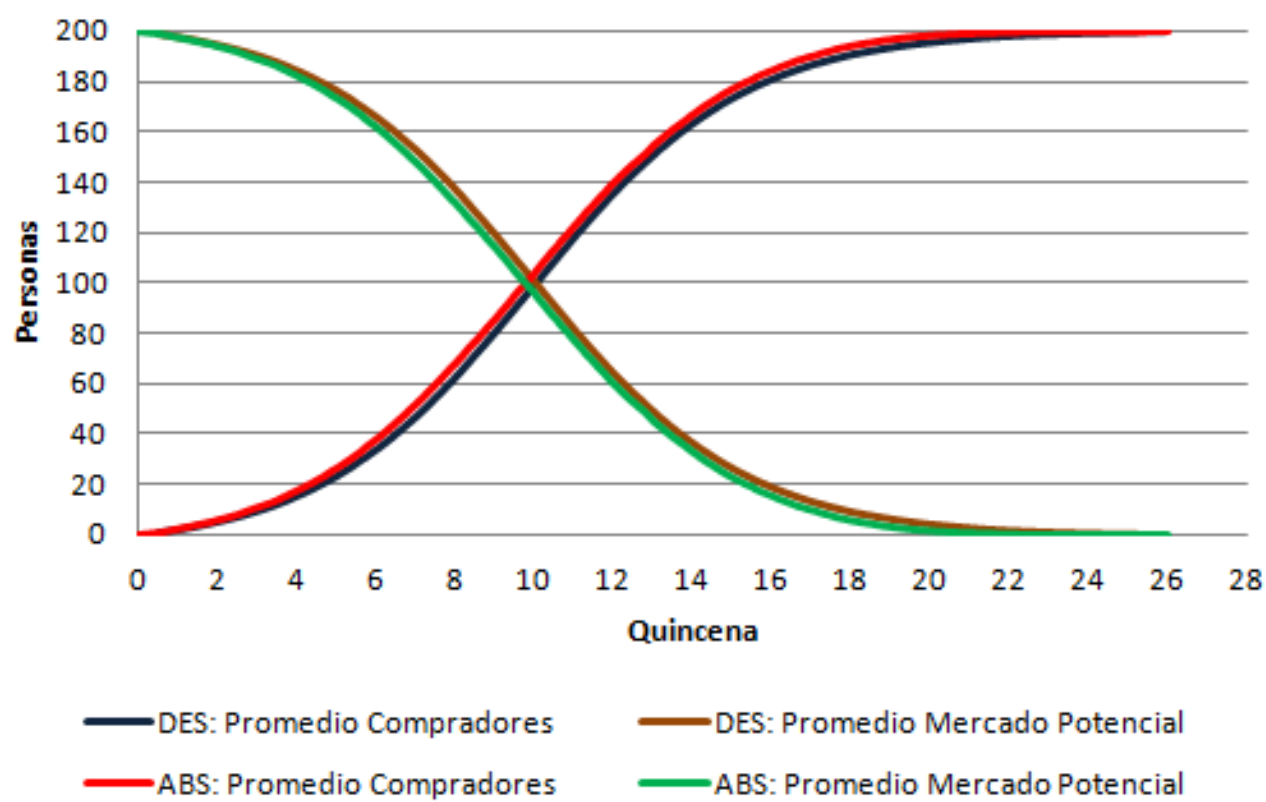

Elaboración propia. 
La Figura 3.22 muestra el comportamiento de la variable Ordenes de Producción generado por el modelo de ABS. Se aprecia que también es bastante similar al generado con el modelo de DES.

Figura 3.22.

Comparación ABS y DES: Ordenes de Producción.

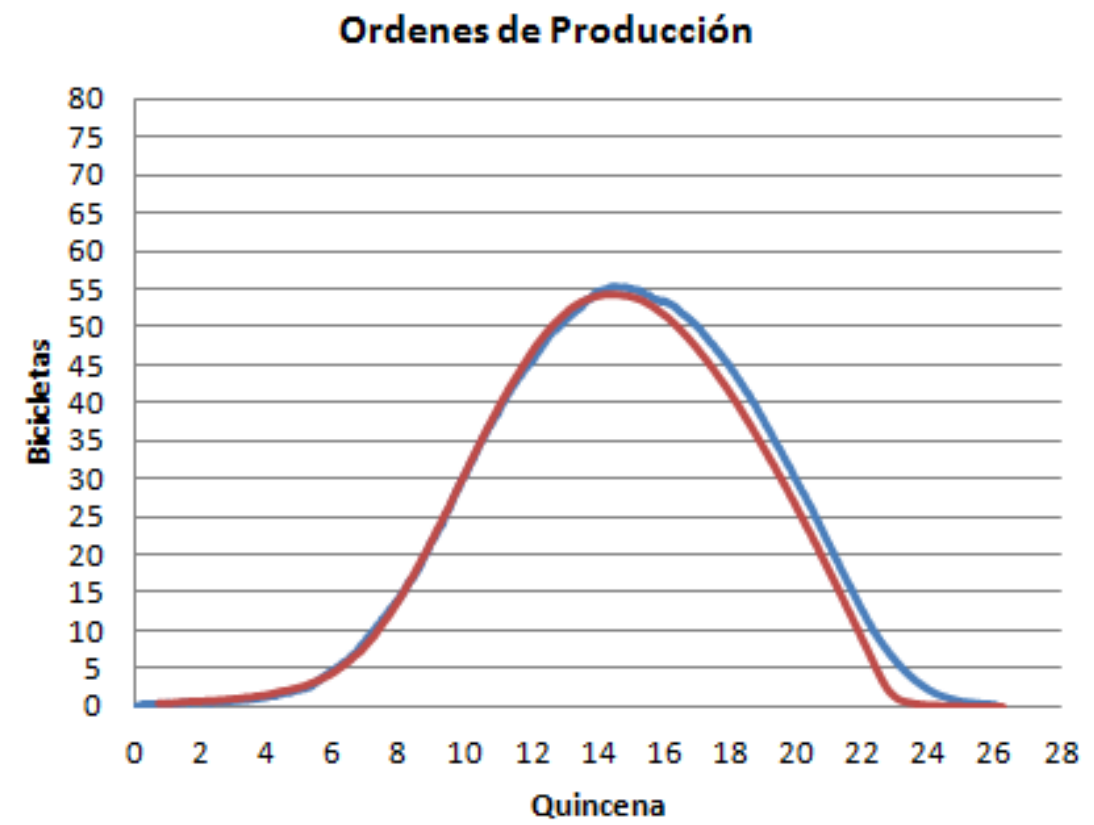

Elaboración propia.

\subsection{Comparación cuantitativa de los paradigmas}

Esto se logra mediante el planteamiento de diversos escenarios independientes que pueden significar cambios en el escenario original descrito para la empresa ESR en las secciones 3.1, 3.2 y 3.3. El hecho que sean escenarios independientes significa que un posible cambio en un escenario no se aplicará a otro escenario, sólo será una modificación de la situación original. El escenario original fue modelado en las secciones $3.4,3.5$ y 3.6 con resultados similares para los tres paradigmas. A partir de ahí, estas nuevos escenarios plantearán retos a cada uno de los paradigmas para adaptar los modelos correspondientes a las nuevas situaciones con el fin de brindar información adicional o proveer capacidad de análisis. 


\subsubsection{Escenario 1: Entendiendo los procesos}

A Clara, la administradora de la empresa, le gustaría saber a cuanto asciende el tiempo promedio de procesamiento de una orden de producción (TPOP) de una bicicleta del modelo VELOZ. El TPOP se toma desde la llegada de la orden de producción hasta el momento en que la bicicleta ya está fabricada (rumbo al inventario de productos terminados). También se desea saber cuanto de ese tiempo total pasa en promedio una orden de producción en la cola. Finalmente se quiere conocer cual es el porcentaje promedio de utilización de las líneas de producción.

\subsubsection{Modelo DES para el Escenario 1}

Estas preguntas son muy fáciles de responder usando DES. ARENA entrega cada una de estas estadísticas en el reporte de resultados consolidados para las 1000 replicaciones, mediante un valor promedio de la estadística y la mitad de la longitud del intervalo de confianza. Solo se deben hacer unas pequeñas modificaciones al modelo original de la Figura 3.4. Estos cambios se muestran en la Figura 3.23.

Figura 3.23.

Modificaciones del modelo original en ARENA para el Escenario 1.

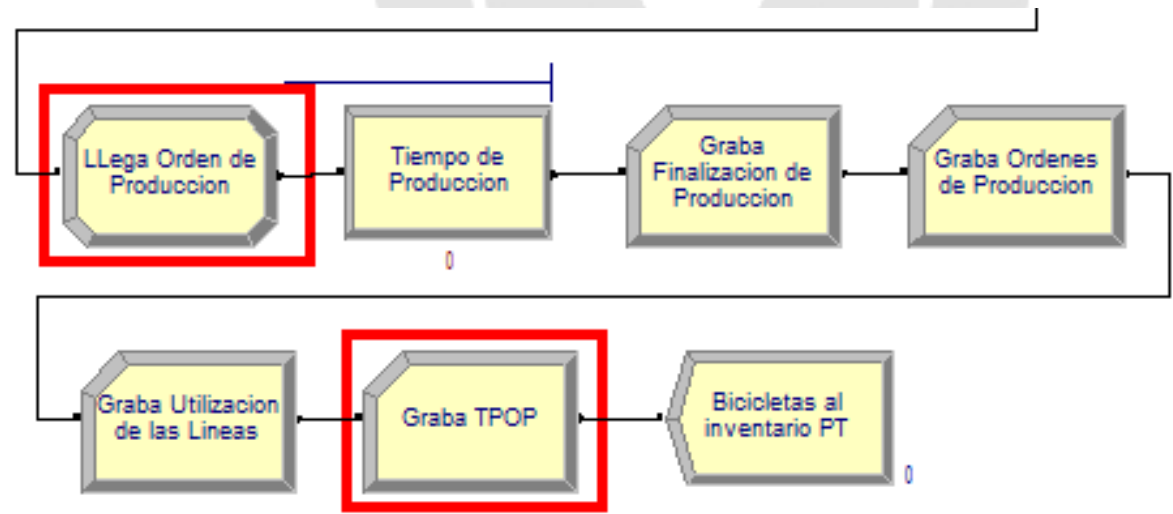

Elaboración propia.

Estas modificaciones consisten en asignar un atributo a cada entidad con el tiempo de llegada de la orden de producción (módulo ASSIGN: Llega Orden de Producción) y restar ese tiempo de llegada del tiempo de finalización de dicha orden (módulo RECORD: Graba TPOP).

El valor promedio del TPOP fue de 2.8 quincenas o 42 días. La mitad de la longitud del intervalo de confianza para dicho porcentaje fue $<0.03$. De este tiempo 
total, una orden pasa 0.2 quincenas (3 días) en promedio en la línea de producción y 2.6 quincenas esperando en la cola. Todos estos tiempos fueron recolectados a nivel de entidad y luego promediados.

El valor promedio del porcentaje de la utilización de las dos líneas de producción para el tiempo total de la simulación fue del 77.35\%. Además, la mitad de la longitud del intervalo de confianza para dicho porcentaje fue $<0.01$.

El nivel de confianza usado para generar los intervalos de todas las estadísticas mostradas en este documento para DES es del 95\% (valor por defecto de ARENA).

Comentario sobre el modelo ABS para este escenario: En este tipo de escenario donde los flujos de las órdenes de producción están claramente delineados y no existen iteraciones complejas entre dichas órdenes entonces se prefiere usar DES sobre ABS. Es decir, el modelo ABS mostraría similares resultados que el modelo DES pero con un mayor esfuerzo computacional.

\subsubsection{Modelo SD para el Escenario 1}

Por otro lado, SD puede no responder a la pregunta del TPOP, ya que como no se representan las entidades en este paradigma, entonces no se tienen estadísticas de lo que toma fabricar cada orden de producción ni cuanto tiempo está cada una de estas órdenes en espera de ser procesada.

Con respecto a la utilización promedio de ambas líneas de producción, se puede calcular del modelo de SD sacando un promedio de las utilizaciones de la siguiente forma:

Utilización Promedio las Líneas $=\frac{\int_{0}^{\mathrm{T}} \mathrm{U}(\mathrm{t}) \mathrm{dt}}{\mathrm{T}}$

donde $\mathrm{U}(\mathrm{t})$ es el valor de la utilización instantánea obtenida de la Figura 3.18 y dt es el intervalo de tiempo que esa utilización estuvo vigente. En el caso del modelo de SD el dt es constante igual al $\Delta \mathrm{t}$ ó TIME STEP definido en VENSIM. Siendo T el tiempo total de la simulación (en VENSIM se define como FINAL TIME), que en este caso fue 26 quincenas.

Esto se puede implementar fácilmente en VENSIM añadiendo unas variables adicionales al modelo como se muestra en la Figura 3.24. 
Figura 3.24.

Modificaciones del modelo original en VENSIM para el Escenario 1.

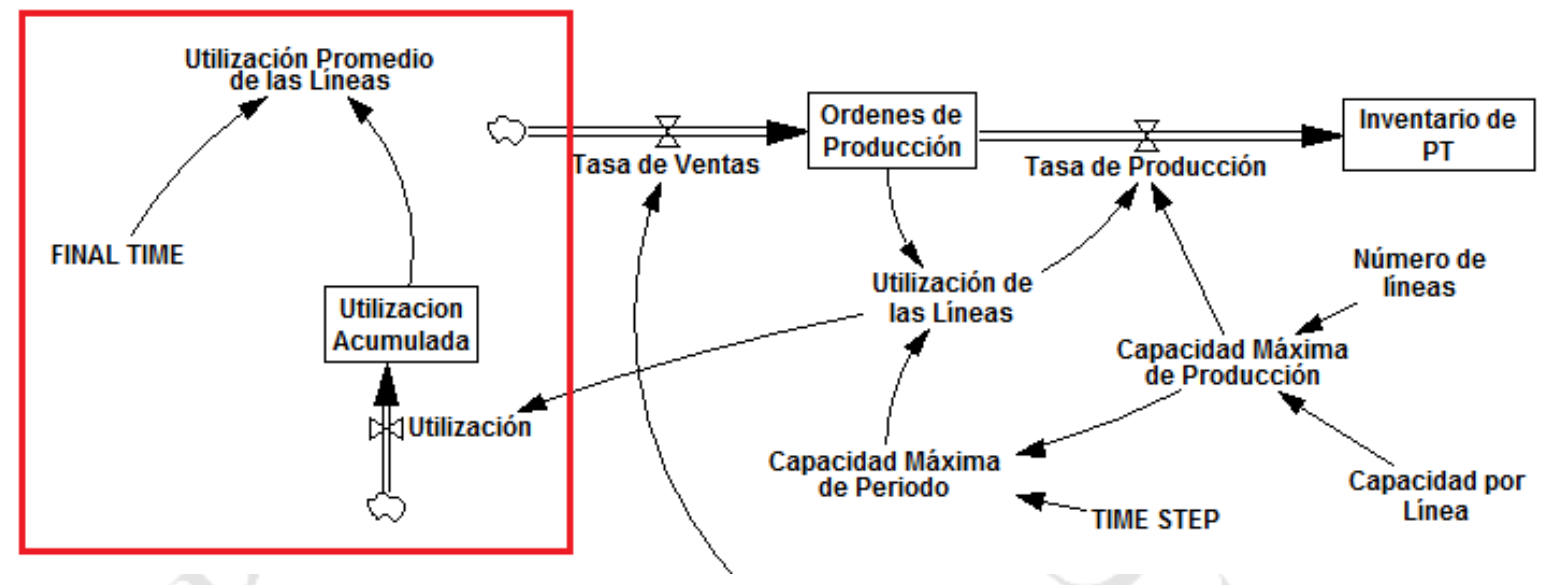

Elaboración propia.

El valor promedio del porcentaje de la utilización de las dos líneas de producción para el tiempo total de la simulación en SD fue del $76.73 \%$. Es un valor aproximado muy cercano al obtenido con el modelo de DES.

\subsubsection{Escenario 2: Entendiendo el mercado}

La gerencia de ESR desea conocer mejor el comportamiento de las ventas. Básicamente quisiera poder responder a dos preguntas:

PREGUNTA 1: ¿Cuáles de las tasas (por publicidad o boca a boca) tienen mayor impacto en el número de compradores?

PREGUNTA 2: Se está planeando hacer una inversión en materiales para poder responder a las ventas correspondientes al 50\% del mercado en las primeras 10.1 quincenas (datos del escenario original). Se desea saber: ¿cuál es la probabilidad de que se alcance una cuota de mercado distinta en las primeras 10.1 quincenas? y ¿cuál es la probabilidad de que se alcance la cuota de $50 \%$ del mercado en un tiempo mayor a menor a las 10.1 quincenas?

\subsubsection{Análisis de las ventas con SD}

Como SD trabaja con valores promedio no puede responder a la PREGUNTA 2 que requiere incluir la aleatoriedad de la variable Compras. Sin embargo, si cuenta con herramientas para poder responder a la PREGUNTA 1. Esto se realiza haciendo un análisis interno del modelo que identifica a los componentes 
(bucles) que influyen en el comportamiento de ciertas variables. El comportamiento de la curva de las ventas que está representada por el valor de la variable Compradores es el resultado de la interacción dinámica (cambia con el tiempo) de los siguientes tres bucles: R3, B5 y B6 que fueron definidos en la sección 3.5. Este análisis busca determinar cuáles son los bucles dominantes responsables por el comportamiento de dicha variable y durante que intervalo de tiempo ejercen ese dominio.

SD tiene varias técnicas para encontrar bucles dominantes (Ford, 1999; Phaff et al., 2006). En este trabajo se utilizará una metodología basada en la primera derivada $(\dot{\mathrm{x}}=\mathrm{dx} / \mathrm{dt})$ y en la segunda derivada $(\mathrm{dx} / \mathrm{dt})$ asociada con una variable objeto de estudio " $\mathrm{x}$ " $\mathrm{y}$ en la interpretación de los siguientes 7 patrones de comportamiento basados en esas derivadas (Mojtahedzadeh et al., 2004): 6 de estos patrones están relacionados con crecimiento y decrecimiento de las curvas (Figura 3.25) y el séptimo patrón es el equilibrio (representado por una línea recta horizontal a lo largo del tiempo).

Figura 3.25.

Seis patrones de comportamiento.

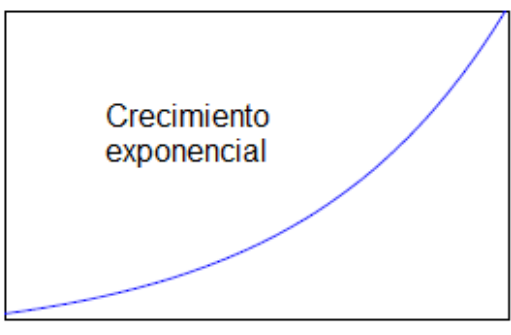

tiempo

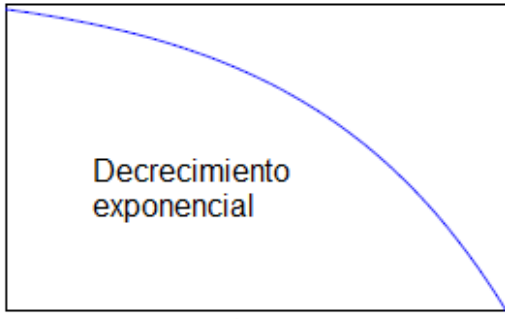

tiempo

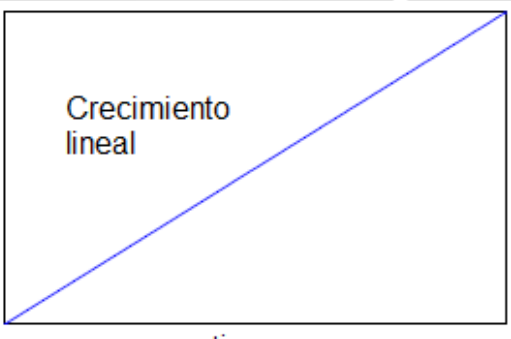

tiempo

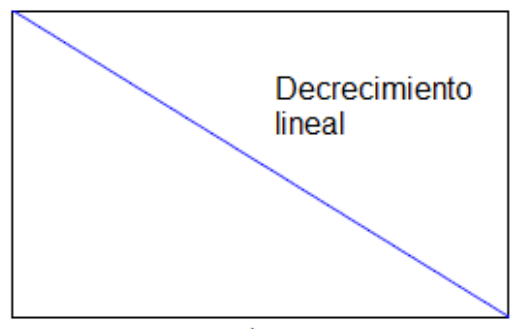

tiempo

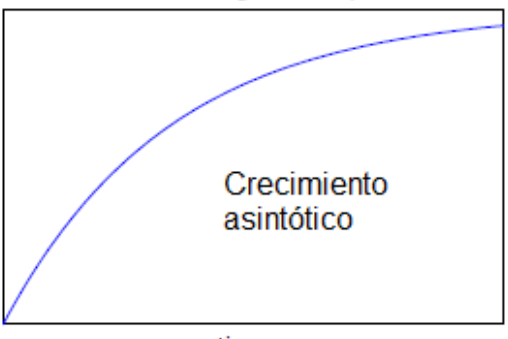

tiempo

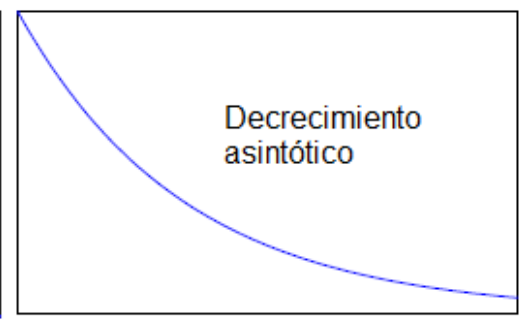

tiempo

Elaboración propia.

De acuerdo a los valores que pueden tomar la primera y segunda derivada a lo largo del tiempo se cumple lo siguiente (Huang, 2012):

- Crecimiento exponencial, $(\dot{\mathrm{x}}>0) \&(\mathrm{~d} \dot{\mathrm{x}} / \mathrm{dt}>0)$

- Decrecimiento exponencial, $(\dot{\mathrm{x}}<0) \&(\mathrm{dx} / \mathrm{dt}<0)$ 
- Crecimiento asintótico, $(\dot{\mathrm{x}}>0) \&(\mathrm{~d} \dot{\mathrm{x}} / \mathrm{dt}<0)$

- Decrecimiento asintótico, $(\dot{\mathrm{x}}<0) \&(\mathrm{~d} \dot{\mathrm{x}} / \mathrm{dt}>0)$

- Crecimiento lineal, $(\dot{\mathrm{x}}>0) \&(\mathrm{~d} \dot{\mathrm{x}} / \mathrm{dt}=0)$

- Decrecimiento lineal, $(\dot{\mathrm{x}}<0) \&(\mathrm{~d} \dot{\mathrm{x}} / \mathrm{dt}=0)$

- Equilibrio, $(\dot{\mathrm{x}}=0) \&(\mathrm{~d} \dot{\mathrm{x}} / \mathrm{dt}=0)$

El algoritmo del análisis para identificar los bucles dominantes sigue los siguientes pasos:

Paso 1. Determinar la variable de estado objeto de estudio (variable de interés).

Paso 2. Definir un intervalo de tiempo para el análisis de la variable de estudio.

Paso 3. Identificar los bucles de realimentación asociados con la variable de estudio y calcular su ganancia.

Paso 4. Identificar los intervalos donde la primera y segunda derivadas de la variable de estudio cambian de signo. En el caso de la segunda derivada, expresarla en función de las ganancias de sus bucles asociados (identificados en el paso 3) con el fin de calcular su signo. A los bucles responsables por el signo de la segunda derivada se les denomina bucles dominantes (Hayward, 2012).

Paso 5. En base a los valores de la primera y segunda derivadas y teniendo en cuenta los siete patrones de comportamiento identificar la forma de la curva de la variable de estudio.

A continuación se aplica el análisis de los bucles dominantes al modelo del pronóstico de la demanda de ESR con el fin de explicar el comportamiento de la variable Compradores.

Paso 1. Se hace que la variable objeto de estudio sea Compradores, la cual se abreviará como "C". Las otras variables se abrevian según se muestra en la Figura 3.26 que representa la parte del modelo de SD asociada con el pronóstico de la demanda. 
Figura 3.26.

Diagrama de Forrester para el pronóstico de la demanda.

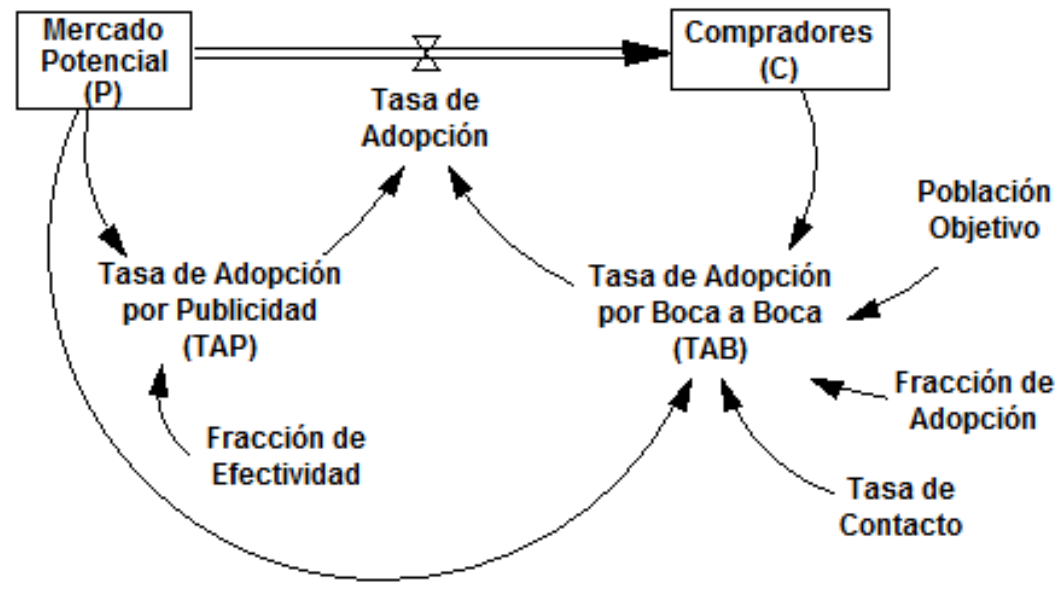

Elaboración propia.

Teniendo como base las ecuaciones del modelo de difusión tecnológica definidas en la sección 3.3.1 y considerando:

- $\quad$ Fracción de Efectividad $=0.01$

- $($ Tasa de Contacto*Fracción de Adopción $) /($ Población Objetivo $)=$ $8 * 0.045 / 200=0.0018$

se expresan las ecuaciones del sistema:

$\mathrm{TAP}=0.01 * \mathrm{P}$

(1)

$\mathrm{TAB}=0.0018 * \mathrm{P} * \mathrm{C}$

$\dot{\mathrm{P}}=-\mathrm{TA}=-\mathrm{TAP}-\mathrm{TAB}=-0.01 * \mathrm{P}-0.0018 * \mathrm{P} * \mathrm{C}$

$\dot{\mathrm{C}}=\mathrm{TA}=\mathrm{TAP}+\mathrm{TAB}=0.01 * \mathrm{P}+0.0018 * \mathrm{P} * \mathrm{C}$

Paso 2. El intervalo de tiempo de análisis será igual al horizonte de tiempo de 26 quincenas para el que se corrió el modelo de SD.

Paso 3. En la sección 3.5.1 se identificó que los bucles que afectan a la variable Compradores son: R3, B5 y B6. Para cada uno de los tres bucles se determina su ganancia G.

$\underline{\text { Para el bucle R3 }}$

$\mathrm{G}(\mathrm{R} 3)=\mathrm{G}(\mathrm{C} \rightarrow \mathrm{TAB} \rightarrow \mathrm{TA})=\mathrm{G}(\mathrm{C} \rightarrow \mathrm{TAB}) * \mathrm{G}(\mathrm{TAB} \rightarrow \mathrm{TA})$ 
$\mathrm{G}(\mathrm{R} 3)=\frac{\mathrm{dTAB}}{\mathrm{dC}} * \frac{\mathrm{dTA}}{\mathrm{dTAB}}=(0.0018 * \mathrm{P}) *(1)=0.0018 \mathrm{P}$

$\underline{\text { Para el bucle B5 }}$

$$
\begin{aligned}
& \mathrm{G}(\mathrm{B} 5)=\mathrm{G}(\mathrm{P} \rightarrow \mathrm{TAP} \rightarrow \dot{\mathrm{C}} \rightarrow \mathrm{C} \rightarrow \mathrm{TAB} \rightarrow \dot{\mathrm{P}}) \\
& \mathrm{G}(\mathrm{B} 5)=\mathrm{G}(\mathrm{P} \rightarrow \mathrm{TAP}) * \mathrm{G}(\mathrm{TAP} \rightarrow \dot{\mathrm{C}}) * \mathrm{G}(\dot{\mathrm{C}} \rightarrow \mathrm{C}) * \mathrm{G}(\mathrm{C} \rightarrow \mathrm{TAB}) * \mathrm{G}(\mathrm{TAB} \rightarrow \dot{\mathrm{P}}) \\
& \mathrm{G}(\mathrm{B} 5)=\frac{\mathrm{dTAP}}{\mathrm{dP}} * \frac{\mathrm{d} \dot{\mathrm{C}}}{\mathrm{dTAP}} * \frac{\mathrm{dC}}{\mathrm{d} \dot{\mathrm{C}}} * \frac{\mathrm{dTAB}}{\mathrm{dC}} * \frac{\mathrm{dP}}{\mathrm{dTAB}} \\
& \mathrm{G}(\mathrm{B} 5)=(0.01) *(1) *\left(\frac{1}{0.0018 \mathrm{P}}\right) *(0.0018 \mathrm{P}) *(-1)=-0.01
\end{aligned}
$$

\section{$\underline{\text { Para el bucle B6 }}$}

$$
\begin{aligned}
& \mathrm{G}(\mathrm{B} 6)=\mathrm{G}(\mathrm{P} \rightarrow \mathrm{TAB} \rightarrow \dot{\mathrm{C}} \rightarrow \mathrm{C} \rightarrow \mathrm{TAB} \rightarrow \dot{\mathrm{P}}) \\
& \mathrm{G}(\mathrm{B} 6)=\mathrm{G}(\mathrm{P} \rightarrow \mathrm{TAB}) * \mathrm{G}(\mathrm{TAB} \rightarrow \dot{\mathrm{C}}) * \mathrm{G}(\dot{\mathrm{C}} \rightarrow \mathrm{C}) * \mathrm{G}(\mathrm{C} \rightarrow \mathrm{TAB}) * \mathrm{G}(\mathrm{TAB} \rightarrow \dot{\mathrm{P}}) \\
& \mathrm{G}(\mathrm{B} 6)=\frac{\mathrm{dTAB}}{\mathrm{dP}} * \frac{\mathrm{d} \dot{\mathrm{C}}}{\mathrm{dTAB}} * \frac{\mathrm{dC}}{\mathrm{d} \dot{\mathrm{C}}} * \frac{\mathrm{dTAB}}{\mathrm{dC}} * \frac{\mathrm{dP}}{\mathrm{dTAB}} \\
& \mathrm{G}(\mathrm{B} 6)=(0.0018 \mathrm{C}) *(1) *\left(\frac{1}{0.0018 \mathrm{P}}\right) *(0.0018 \mathrm{P}) *(-1)=-0.0018 \mathrm{C}
\end{aligned}
$$

Paso 4. Se calcula la primera y segunda derivada de la variable objeto de estudio "C", es decir, $(\dot{C}=d C / d t)$ y $(d \dot{C} / d t)$. Pero la primera derivada ya fue calculada en la ecuación (4):

$$
\dot{\mathrm{C}}=\mathrm{TA}=0.01 * \mathrm{P}+0.0018^{*} \mathrm{P} * \mathrm{C}
$$

y por la Figura 3.16 (ya que $\dot{C}$ es igual a la tasa de adopción) se sabe que es positiva para el periodo de tiempo de la simulación que es 26 quincenas, es decir, $\dot{\mathrm{C}}>0, \forall \mathrm{t} \in[0,26]$. La segunda derivada se obtiene derivando la ecuación (5) respecto al tiempo:

$\ddot{\mathrm{C}}=0.01 * \dot{\mathrm{P}}+0.0018 * \dot{\mathrm{P}} * \mathrm{C}+0.0018 * \mathrm{P} * \dot{\mathrm{C}}$

$\ddot{\mathrm{C}}=(0.01+0.0018 \mathrm{C}) * \dot{\mathrm{P}}+0.0018 * \mathrm{P} * \dot{\mathrm{C}}$

Se multiplica y divide por $\dot{\mathrm{C}}$

$$
\ddot{\mathrm{C}}=\left((0.01+0.0018 \mathrm{C}) * \frac{\dot{\mathrm{P}}}{\dot{\mathrm{C}}}+0.0018 * \mathrm{P} * \frac{\dot{\mathrm{C}}}{\dot{\mathrm{C}}}\right) \dot{\mathrm{C}}
$$


Pero dividiendo la ecuación (3) entre (4) se tiene que $\frac{\dot{\mathrm{P}}}{\dot{\mathrm{C}}}=-1$

$\ddot{\mathrm{C}}=(-0.01-0.0018 \mathrm{C}+0.0018 \mathrm{P}) \dot{\mathrm{C}}$

Expresando la segunda derivada en función de las ganancias de los bucles:

$\ddot{\mathrm{C}}=(\mathrm{G}(\mathrm{B} 5)+\mathrm{G}(\mathrm{B} 6)+\mathrm{G}(\mathrm{R} 3)) \dot{\mathrm{C}}$

Como $\dot{\mathrm{C}}>0$ solo de debe analizar el término $\mathrm{GNETA}=(\mathrm{G}(\mathrm{B} 5)+\mathrm{G}(\mathrm{B} 6)+\mathrm{G}(\mathrm{R} 3)$ para saber el signo de la segunda derivada en el intervalo de tiempo [0,26].

En la Figura 3.27 se aprecia que la ganancia de los bucles B5 y B6 es negativa mientras que la del bucle R3 es positiva para todo el intervalo de tiempo $[0,26]$.

Figura 3.27.

Ganancia de los bucles.

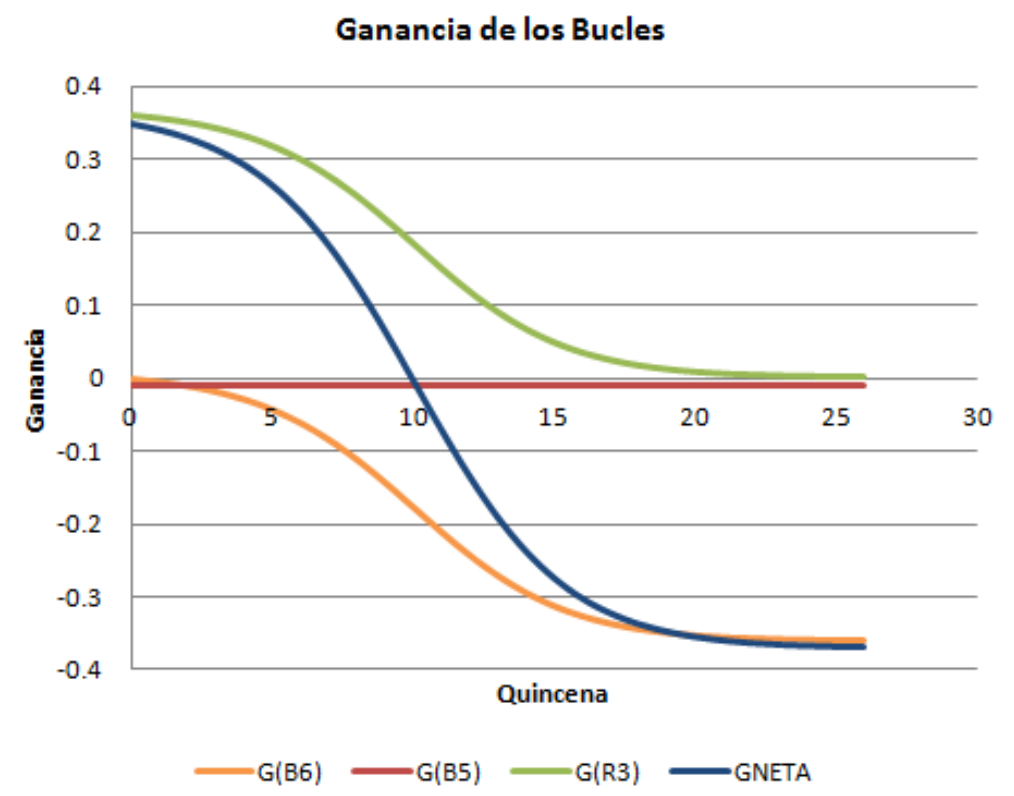

Elaboración propia.

Por lo tanto como la GNETA es positiva en el intervalo [0,10], se debe a la mayor ganancia del bucle R3 y por lo tanto este bucle domina el comportamiento de la variable Compras en las primeras 10 quincenas. La G(R3) depende de "P", el tamaño del mercado potencial, que al comienzo es mucho mayor que el número de compradores y por eso el dominio de este bucle.

Luego la GNETA toma valores negativos en el intervalo $<10,26]$ indicado que los bucles B5 y B6 dominan el comportamiento de la variable Compras las últimas 16 quincenas. Realmente el aporte del bucle B5 es muy pequeño y quien domina las 
últimas 16 quincenas es el bucle B6. La G(B6) depende de "C", el número de compradores, que al comienzo es mucho menor que el tamaño del mercado potencial pero que luego lo supera ampliamente y por eso el dominio de este bucle.

Paso 5. En el intervalo $[0,10]$ al ser la primera y segunda derivadas positivas entonces corresponde a un comportamiento de crecimiento exponencial de las compras (ver criterios de la primera y segunda derivada para los siete patrones). En el intervalo $<10,26$ ] al ser la primera derivada positiva y la segunda derivada negativa entonces corresponde a un comportamiento de crecimiento asintótico de las compras (ver Figura $3.28)$.

Figura 3.28.

Comportamiento de la curva Compradores.

\section{Compradores}

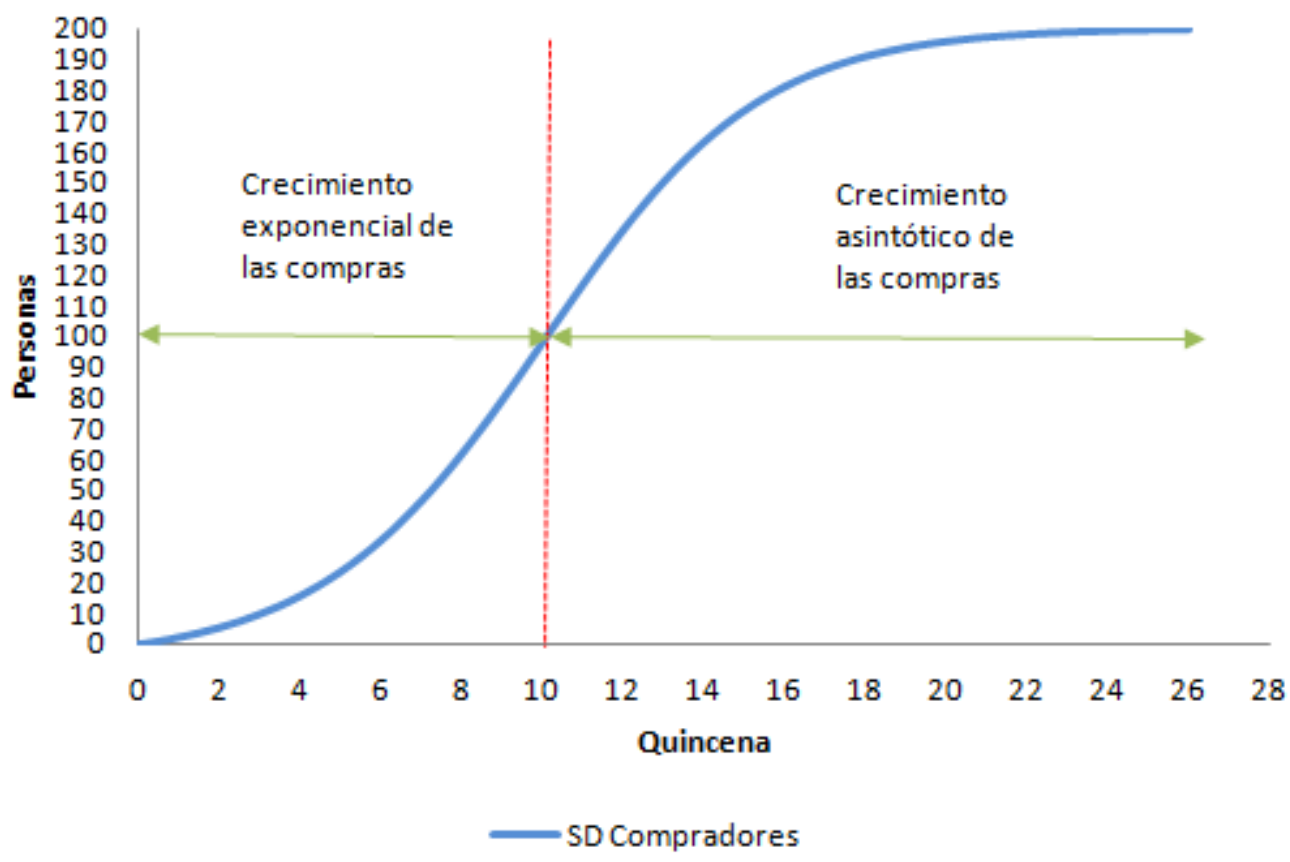

Elaboración propia.

Con los parámetros actuales del modelo, se puede concluir que la Tasa de Adopción Boca a Boca participa activamente en los dos bucles dominantes (R3 y B6) que marcan el comportamiento de las ventas. Por otro lado, la Tasa de Adopción por Publicidad ayuda a inicializar las ventas (deben haber ventas iniciales para que permitan el desarrollo del efecto boca a boca) pero luego su efecto es secundario con respecto al del boca a boca. Este análisis permite dar una respuesta a las PREGUNTA 1. 


\subsubsection{Análisis de las ventas con DES}

Aunque el propósito principal de DES no está en encontrar relaciones de causalidad al interior del sistema que expliquen el comportamiento de ciertas variables, como es el caso de SD, se puede hacer uso de la teoría estadística para llegar a ciertas conclusiones.

La forma de modelamiento en DES no provee de un entorno gráfico que muestre fácilmente las relaciones de causalidad. Aunque en ciertos casos estas relaciones puedan estar definidas explícitamente, en modelos muy grandes con muchos módulos es bastante complicado hallar la interrelación entre ellas. Además el hecho de que DES trabaje con variables aleatorias hace más difícil determinar si el comportamiento de una variable (cambio de sus valores) se debe a la influencia de otra variable o al efecto de la aleatoriedad.

Debido a ello, en DES no se trabaja con el concepto de causalidad sino más bien con el concepto de correlación. La correlación es una relación estadística entre dos o más variables. Mientras que la causalidad es una relación entre un evento (la causa) y un segundo evento (el efecto), donde el segundo evento es una consecuencia del primero (Ramírez, 2014). Por lo tanto, la correlación no necesariamente implica causalidad.

Como medida de fuerza en la relación de las variables se utiliza el coeficiente de correlación (R), el cual se define para dos variables "x" e "y" de la siguiente forma:

$$
\mathrm{R}=\frac{\sum_{\mathrm{i}=1}^{\mathrm{n}}\left(\mathrm{x}_{\mathrm{i}}-\overline{\mathrm{x}}\right)\left(\mathrm{y}_{\mathrm{i}}-\overline{\mathrm{y}}\right)}{\sqrt{\sum_{\mathrm{i}=1}^{\mathrm{n}}\left(\mathrm{x}_{\mathrm{i}}-\overline{\mathrm{x}}\right)^{2}} \sqrt{\sum_{\mathrm{i}=1}^{\mathrm{n}}\left(\mathrm{y}_{\mathrm{i}}-\overline{\mathrm{y}}\right)^{2}}}
$$

El problema es que este coeficiente está definido para encontrar relaciones lineales entre las variables y falla cuando existe relaciones no lineales como es el caso del modelo Bass, donde la Tasa de Adopción por Boca a Boca se define como el producto de las variables Mercado Potencial y Compradores. Esto queda verificado al calcular dicho coeficiente para encontrar la correlación entre la variable Tasa de Adopción (que incluye a la Tasa de Adopción por Boca a Boca) y la variable Compradores. Se usan los 200 pares de datos $\left(\mathrm{TA}_{\mathrm{t}}, \mathrm{C}_{\mathrm{t}}\right)$ obtenidos de correr el modelo DES.

Coeficiente de correlación $(\mathrm{R})=-0.13$ 
Un valor negativo de $\mathrm{R}$ indica que al incrementarse la tasa de adopción entonces el número de compradores disminuye. Lo cual está equivocado, ya que un incremento de la tasa de adopción genera un incremento en el número de compradores. Además para justificar que haya fuerte correlación entre ambas variables se debe cumplir que $\mathrm{R}^{2} \geq 0.5$ (Taylor, 1990), lo cual tampoco se da al ser $\mathrm{R}^{2}=0.017$.

Queda en evidencia que DES no puede responder de manera satisfactoria a la PREGUNTA 1. Sin embargo, al incluir el modelo DES el factor de aleatoriedad de las variables, sí se puede responder a la PREGUNTA 2. Esto se muestra en los siguientes párrafos.

La Figura 3.29 muestra las curvas correspondientes a los valores promedio de la variable Compradores así como las curvas correspondientes a los percentiles 5th y 95th de dicha variable.

Figura 3.29.

Curva de percentiles: análisis horizontal.

\section{Compradores}

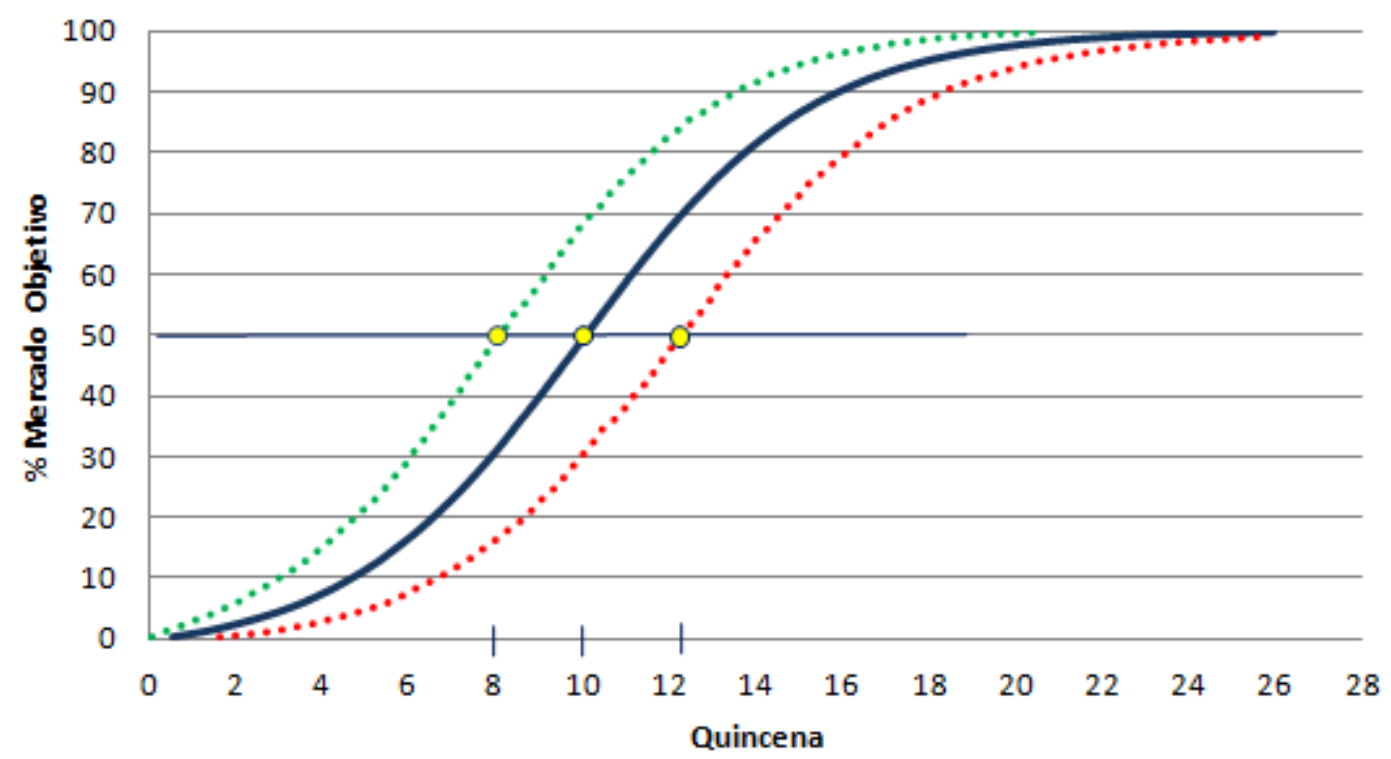

- DES: Promedio $\quad \ldots \ldots$ DES: 5 th perc. $\quad \ldots \ldots$ DES: 95 th perc.

Elaboración propia.

En el eje de las ordenadas se tiene el porcentaje del mercado capturado, es decir, el número de compradores que han adquirido el producto en un momento determinado dividido entre el tamaño total de la población objetivo (que corresponde a 200 personas). Una línea horizontal ha sido trazada en el punto equivalente al $50 \%$ del 
mercado objetivo y se intercepta con las tres curvas generando tres puntos. El primer punto corresponde al percentil 5th, el cual significa que existe una probabilidad de 0.05 de que el tiempo para alcanzar el 50\% de la población objetivo (100 compradores) sea menor o igual a 8.1 quincenas. El segundo punto es la media que indica que el tiempo promedio para alcanzar el 50\% de la población objetivo es de 10.1 quincenas. El tercer punto corresponde al percentil 95th, el cual significa que existe una probabilidad de 0.95 de que el tiempo para alcanzar el $50 \%$ de la población objetivo sea menor o igual a 12.3 quincenas. Este último punto también se puede interpretar como que existe una probabilidad de 0.05 (1-0.95) de que el tiempo para alcanzar el 50\% de la población objetivo sea mayor a 12.3 quincenas.

Por lo tanto, si planificamos con un tiempo de 10.1 quincenas (dado por la curva promedio) para alcanzar el 50\% del mercado, puede suceder con un 5\% de probabilidad de que se alcance 2 quincenas antes (quincena 8.1) o con un 5\% de probabilidad de que realmente se alcance aproximadamente 2 quincenas después (quincena 12.3).

Otro análisis es el que se muestra en la Figura 3.30 y es el que va a responder al otro aspecto de la segunda pregunta planteada por la gerencia de ESR.

Figura 3.30.

Curva de percentiles: análisis vertical.

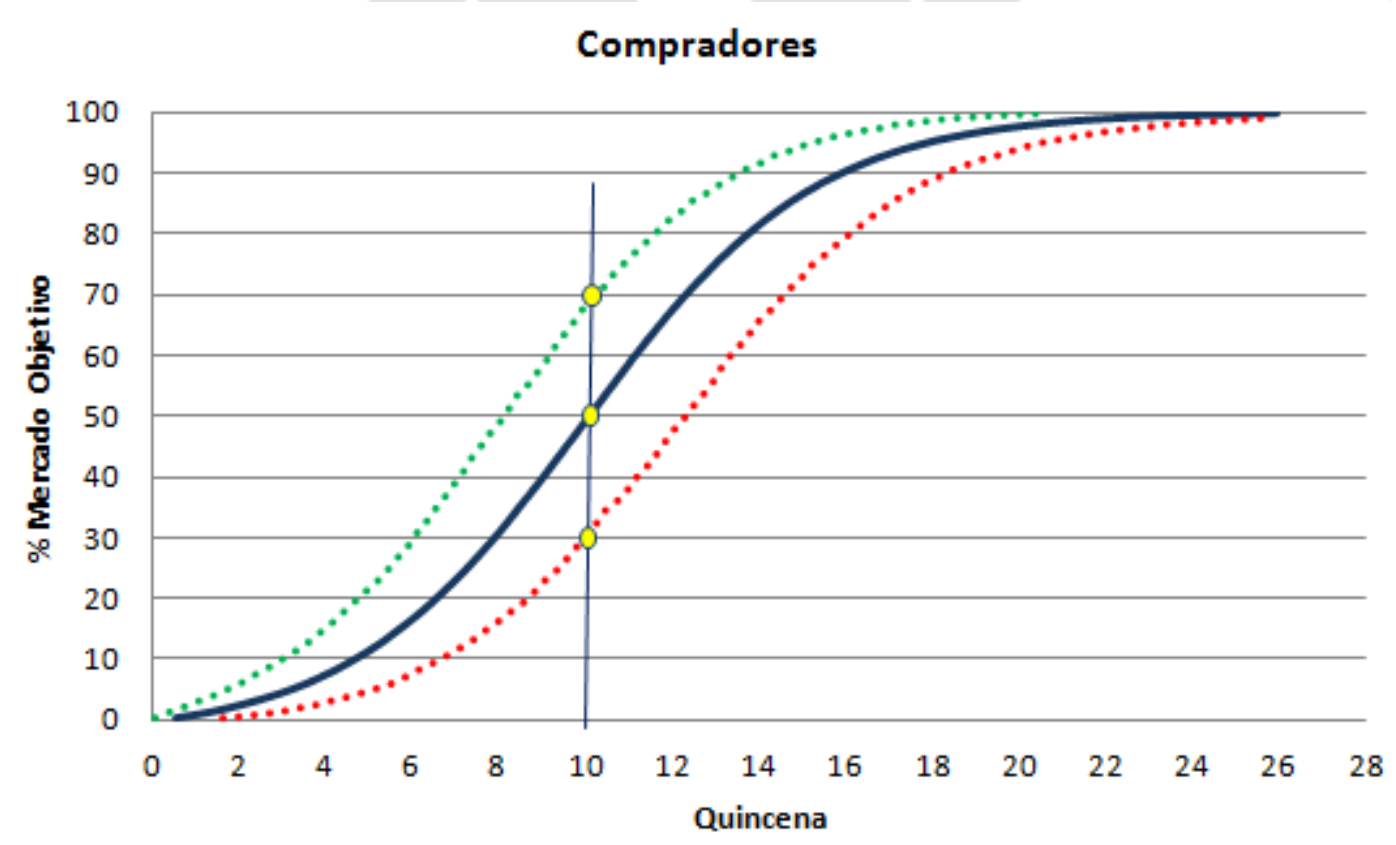

DES: Promedio $\ldots \ldots$ DES: 5 th perc. $\quad \ldots \ldots$ DES: 95 th perc.

Elaboración propia. 
La línea vertical que parte de la quincena 10.1 se intercepta con las tres curvas generando tres puntos. El primer punto corresponde al percentil 5th, el cual significa que existe una probabilidad de 0.05 de alcanzar más del $70 \%$ de la población objetivo en la quincena 10.1. El segundo punto dice que se alcanzará la mitad de la población objetivo en un tiempo promedio de 10.1 quincenas. El tercer punto corresponde al percentil 95th, el cual significa que existe una probabilidad de 0.95 de alcanzar más del $30 \%$ de la población objetivo en la quincena 10.1. Este último punto también se puede interpretar como que existe una probabilidad de 0.05 (1-0.95) de que se alcance menos del $30 \%$ de la población objetivo en la quincena 10.1 .

En conclusión, si se planifica hacer una inversión de materiales como para responder a la demanda del $50 \%$ del mercado en las 10.1 primeras quincenas, existe una probabilidad de 0.05 de que la demanda sea mayor al $70 \%$ y se generen faltantes de materia prima y una probabilidad de 0.05 de que la demanda sea menor al $30 \%$ del mercado y se generen excedentes y costos de almacenamiento.

Dependerá del nivel de servicio (tiempo de entrega) que la gerencia de ESR quiera mantener para tomar la decisión de hacer la inversión planeada.

Comentario sobre el modelo ABS para este escenario: En este tipo de escenario donde los agentes representan a personas homogéneas con los mismos parámetros asociados a todas ellas (probabilidades de adopción) entonces se prefiere usar un modelo DES que requiere de menos cálculos para obtener los mismos resultados que el modelo ABS.

\subsubsection{Escenario 3: Masificación del producto}

Debido a la evolución de la tecnología de las bicicletas eléctricas, éstas han bajado drásticamente sus costos de fabricación, permitiendo con ello la venta masiva de este medio de transporte. Se desea conocer como afectará las operaciones de ESR una ampliación del mercado constituido ahora por una población objetivo de 10 mil personas para el modelo VELOZ. Considerar que la Fracción de Adopción en este nuevo mercado es ahora $0.01 \mathrm{y}$ la Tasa de Contacto es de 4 personas por quincena. Al ser un mercado masivo las personas no comparten los eventos de intereses comunes que se tenían en un mercado más selecto y mucho más pequeño. El contacto de las personas 
objetivo se realiza en actividades comunes del día a día donde ellas están inmersas dentro de la población arequipeña de aproximadamente 500 mil personas.

ESR ha pensado trabajar dos turnos adicionales, es decir, ampliar su capacidad máxima de producción por línea a 15 bicicletas por quincena (en 3 turnos). También se quiere ampliar sus líneas de producción y se desea saber para diferentes alternativas de números de líneas de producción a cuanto llegará el número máximo de órdenes de producción en espera de ser procesadas, el tiempo que tomará procesar todas las órdenes y la utilización de las líneas de producción. Considerar que el tiempo de producción del modelo VELOZ no ha cambiado.

Este escenario es más adecuado de simular con SD en términos de tiempo de simulación, que le toma prácticamente el mismo tiempo simular el modelo para una población objetivo de 200 personas ó de 10 mil personas. En este caso de estudio de ESR el modelo DES es muy sencillo, pero simular miles de entidades en un modelo más complejo con muchas actividades y recursos y además correr 1000 replicaciones en cada alternativa de las varias que se pretende evaluar, puede tomar muchas horas de simulación. Hay técnicas basadas en simulación paralela que permiten aumentar la capacidad de procesamiento de los modelos de DES (Bagrodia et al., 1999; Milivojević et al., 2009).

Definitivamente este escenario tampoco es un caso favorable para usar el paradigma ABS debido a que requiere un mayor esfuerzo computacional que DES de varios millones de iteraciones.

Por lo tanto, usando el modelo de SD de la Figura 3.14 y cambiando solamente los parámetros:

Población Objetivo $=10000$

Tasa de Contacto $=4$

Fracción de Adopción = 0.01

Capacidad por Línea $=15$

se puede responder muy rápidamente a las siguientes preguntas:

a) ¿Cuánto tiempo tomará la saturación del mercado potencial? y ¿cuándo se alcanza el 50\% del mercado? 
Las respuesta es aproximadamente 129 quincenas o 5.4 años (Ver Figura 3.31). Por otro lado, toma 36 quincenas capturar el 50\% del mercado potencial (punto de intersección). Obtener el 50\% restante del mercado toma un tiempo mucho mayor de 93 quincenas ya que el efecto de la publicidad ha disminuido y el efecto del boca a boca sobre las ventas es mucho menor.

Figura 3.31.

Mercado Potencial y Compradores para el Escenario 3.

\section{Compradores y Mercado Potencial}

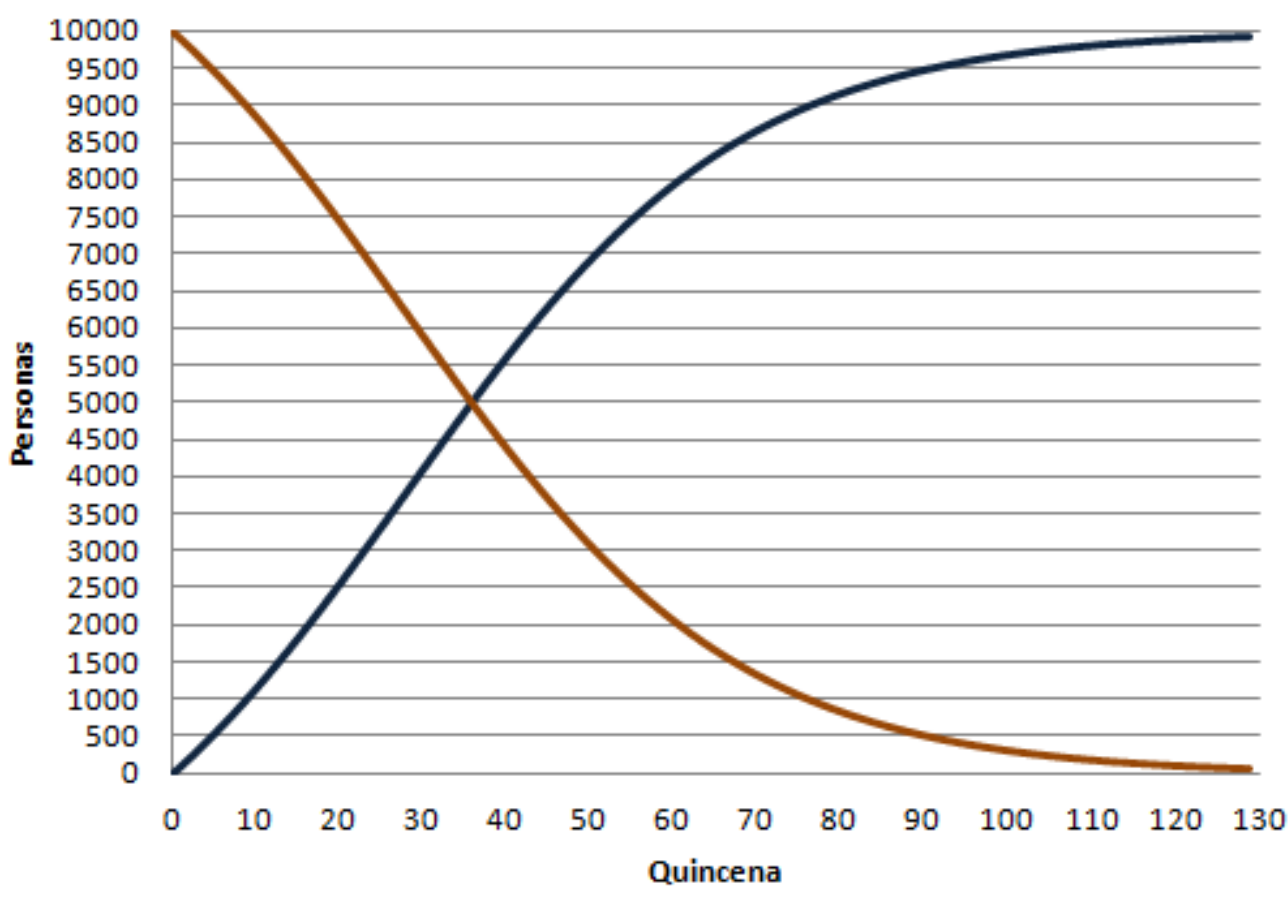

SD: Compradores $\longrightarrow$ SD: Mercado Potencial

Elaboración propia.

b) ¿En qué momento la tasa de adopción llega a su máximo valor y a partir de allí empieza a decrecer?

El valor del tiempo de inflexión es de 27.75 quincenas (ver Figura 3.32). Al comienzo, la Tasa de Adopción por Publicidad con un ratio inicial de 100 bicicletas por quincena es la que contribuye mayormente a las compras del modelo VELOZ. La Tasa de Adopción alcanza su nivel más alto de 156.25 bicicletas por quincena en el tiempo de inflexión.

En este caso $\mathrm{p}=0.01$ y $\mathrm{q}=0.04$ siendo $\mathrm{q} / \mathrm{p}=4$. Este cociente que no es un valor muy grande, contribuye a que haya una diferencia entre el tiempo de inflexión y el tiempo de intersección de 8.25 quincenas (36-27.75). A diferencia del escenario original 
donde ambos tiempos eran casi iguales. Pero en ese caso el cociente de $\mathrm{q} / \mathrm{p}$ era 9 veces mayor al actual (Ver sección 3.4).

Figura 3.32.

Tasa de Adopción para el Escenario 3.

Tasa de Adopción

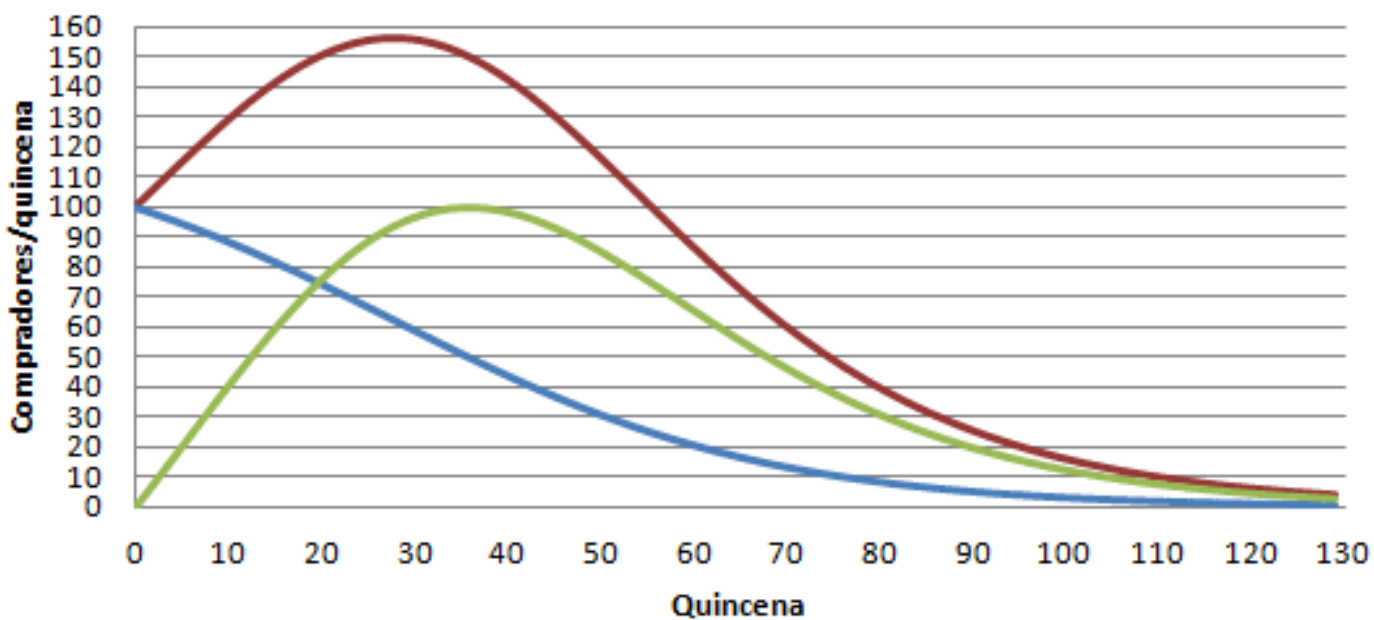

SD: Tasa de Adopción

SD: Tasa Adopción por Publicidad

SD: Tasa de Adopción por Boca a Boca

Elaboración propia.

c) ¿A cuánto ascenderá el máximo nivel acumulado de órdenes en espera de ser procesadas?

Probando para diferentes número de líneas de producción se obtiene la siguiente tabla:

Tabla 3.3. Nivel Acumulado de Ordenes de Producción para diversas Líneas de Producción

\begin{tabular}{|c|c|c|c|}
\hline $\begin{array}{c}\text { Número de } \\
\text { Líneas }\end{array}$ & $\begin{array}{c}\text { Número de } \\
\text { Ordenes }\end{array}$ & $\begin{array}{c}\text { Porcentaje de } \\
\text { Utilización }\end{array}$ & $\begin{array}{c}\text { Tiempo de } \\
\text { Procesamiento Total } \\
\text { (Años) }\end{array}$ \\
\hline 2 & $6,788.6$ & 99.87 & 13.9 \\
\hline 4 & $4,471.7$ & 99.83 & 7.0 \\
\hline 6 & $2,549.8$ & 85.77 & 5.4 \\
\hline 8 & $1,009.3$ & 64.33 & 5.4 \\
\hline 9 & 463.8 & 57.18 & 5.4 \\
\hline 10 & 104.7 & 51.46 & 5.4 \\
\hline 12 & 39.1 & 42.88 & 5.4 \\
\hline
\end{tabular}

Elaboración propia. 
La primera columna muestra las alternativas de implementar distintos números de líneas de producción, la segunda columna muestra el número máximo de órdenes de producción en espera de ser procesadas, la tercera columna muestra el porcentaje de utilización de las líneas y finalmente la cuarta columna muestra el tiempo de procesamiento total por las 10 mil órdenes de producción.

Incrementar más de 6 líneas de producción no ayuda a reducir el tiempo de procesamiento total de 5.4 años debido al comportamiento de las ventas que aún generan órdenes de producción en el año 5.

\subsubsection{Escenario 4: Trabajando dos modelos de bicicletas}

Adicional al modelo VELOZ, ESR decide introducir un segundo modelo de bicicleta denominado STEALTH, dirigido especialmente a personas que hacen ciclismo de montaña. El costo de este nuevo modelo es de 7000 dólares. El marco de titanio usado en el modelo VELOZ es reemplazado por un marco de fibra de carbono. Este nuevo material aporta una mayor comodidad y maniobrabilidad y absorbe mucho mejor las vibraciones en terrenos difíciles. Complementado con una doble suspensión que le da tracción superior a la bicicleta y la posibilidad de ir velocidades mucho más altas en los terrenos más escabrosos.

Adicionalmente este modelo recarga su batería mediante una nueva tecnología que lleva paneles solares en las ruedas. Con buena iluminación podría recargar la batería en aproximadamente 5 horas.

De forma similar al modelo VELOZ, la fabricación del marco se inicia cuando llega la orden de producción y el resto de componentes está listo para ser ensamblado cuando se termina la fabricación del marco.

Con respecto a los tiempos de fabricación de ambos modelos, el del modelo VELOZ no ha cambiado, se mantiene en $(2+\operatorname{NORM}(1,0.5)) / 15$ quincenas. Con un tiempo promedio de 3 días para producir una bicicleta.

Sin embargo, debido a las nuevas características innovadoras del modelo STEALTH su tiempo de fabricación es mayor: (7+NORM $(1,0.5))$ días ó $(7+\operatorname{NORM}(1,0.5)) / 15$ quincenas. Con un tiempo promedio de 8 días para producir una bicicleta. Donde NORM(1,0.5) es una variable aleatoria con una distribución normal con media de 1 día y desviación estándar de 0.5 días. 
Se ha estimado que un $20 \%$ del mercado potencial de 200 personas comprará el modelo STEALTH y el resto continuará comprando el modelo VELOZ. Se mantienen todos los parámetros del modelo de Bass del escenario original. En la planta, existen las dos líneas de producción y además la regla de procesamiento de las órdenes es FIFO (First In First Out), es decir, se procesan en el orden de llegada, independientemente del modelo de bicicleta asociado con la orden.

\subsubsection{Modelo DES para el Escenario 4}

En este modelo el $20 \%$ de las órdenes de producción asociadas con el nuevo modelo son seleccionadas aleatoriamente de las 200 órdenes totales que van llegando a ESR. Esto se hace con la función de ARENA denominada DISC en el módulo ASSIGN: "Definir Tiempos de Producción" que se muestra en la Figura 3.33.

Figura 3.33.

Modificaciones del modelo original en ARENA para el Escenario 4.

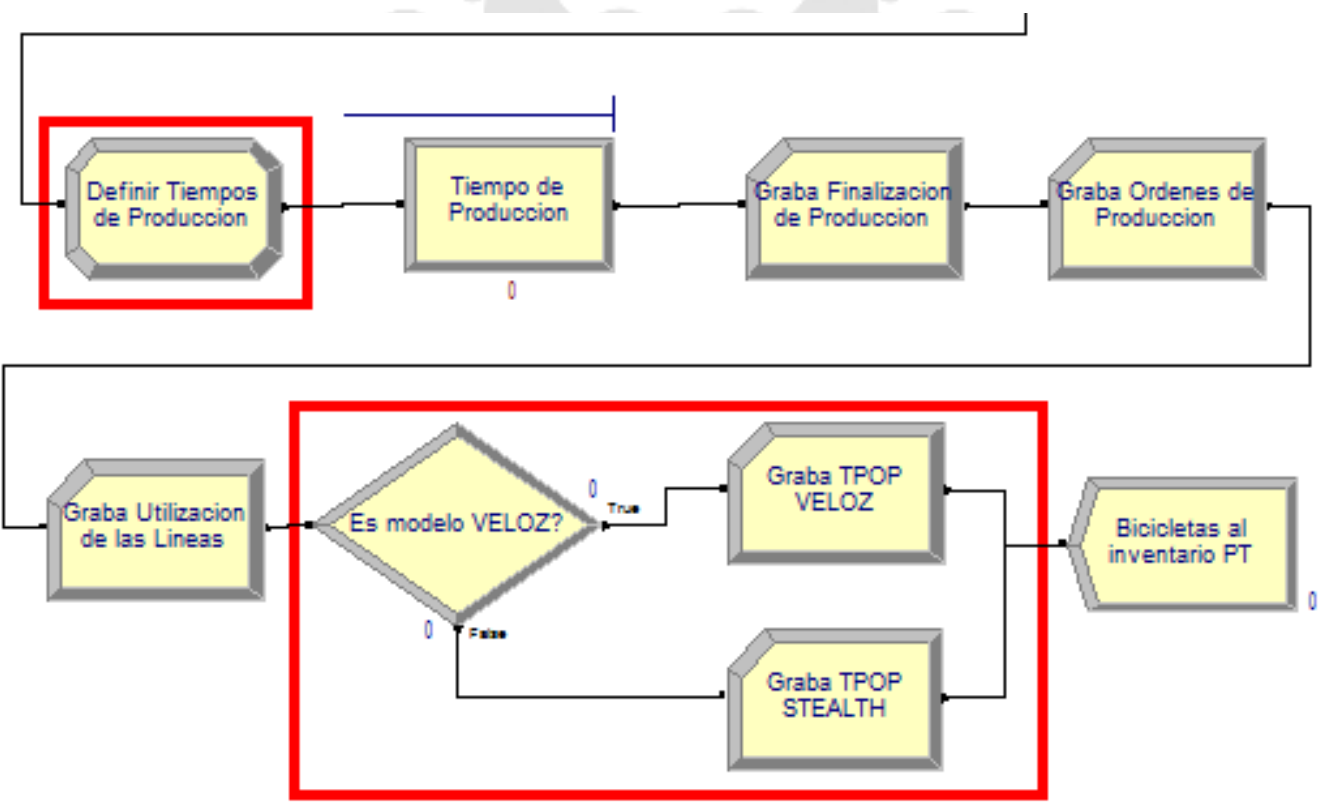

Elaboración propia.

En ese mismo módulo se asigna un atributo a cada entidad con el tiempo de llegada de la orden de producción y que se restará del tiempo de finalización de dicha orden (módulos RECORD: Graba TPOP VELOZ y Graba TPOP STEALTH).

Comentario sobre el modelo ABS para este escenario: En este escenario el manejo de las prioridades de las órdenes de producción es el mismo para ambos modelos DES y ABS. Sin embargo, la generación de dichas órdenes a través de la 
representación de la actividad de compra es más sencillo de hacer con el paradigma DES que sólo requiere 200 entidades en lugar de las 20800 iteraciones de ABS. Por lo tanto, se prefiere el uso de DES sobre ABS en este caso.

\subsubsection{Modelo SD para el Escenario 4}

Como SD no puede representar de manera independiente las órdenes de producción de los modelos VELOZ y STEALTH entonces se debe representar un modelo PROMEDIO de bicicleta que tenga un tiempo de fabricación que sea el promedio de los tiempos de fabricación de cada modelo. Este tiempo se obtiene de la siguiente forma:

Tiempo modelo PROMEDIO $=$ Tiempo promedio modelo VELOZ $* 0.80+$ Tiempo promedio modelo STEALTH * 0.20

Tiempo modelo PROMEDIO $=3$ días $* 0.80+8$ días $* 0.20=4$ días $=0.267$ quincenas por bicicleta

Por lo tanto, la tasa de producción asociada con este modelo PROMEDIO es de 3.75 bicicletas por quincena.

El modelo de SD del escenario original se mantiene en este escenario y lo único que cambia es el valor de la Capacidad por Línea que ahora es 3.75.

Luego de correr los modelos DES y SD se muestra en la Figura 3.34 la curva de las Ordenes de Producción. Se aprecia que ambos modelos arrojan resultados similares, donde las órdenes acumuladas alcanzan su valor máximo (79 órdenes el modelo DES y 77.5 el modelo SD) en la quincena 15.7. Además, toma 30 quincenas terminar de procesar todas las 200 órdenes. Estos son valores mayores a los obtenidos en el escenario original debido al mayor tiempo de producción del modelo STEALTH.

El modelo DES brinda una información adicional que el modelo SD no puede dar. Con 1000 replicaciones, el valor promedio del TPOP para el modelo VELOZ fue de 5.6 quincenas o 84 días. La mitad de la longitud del intervalo de confianza para dicho valor fue $<0.05$. Además, el valor promedio del TPOP para el modelo STEALTH fue de 5.8 quincenas o 87 días. La mitad de la longitud del intervalo de confianza para dicho valor fue $<0.05$. Definitivamente el tiempo de fabricación mucho mayor del modelo STEALTH ha afectado el tiempo de procesamiento de las órdenes de producción del modelo VELOZ por tener ahora mayores esperas. Se aprecia que dos 
líneas de producción son insuficientes para procesar las órdenes de producción en tiempos razonables similares a los del escenario original.

Figura 3.34.

Comparación SD y DES: Ordenes de Producción regla FIFO.

\section{Ordenes de Producción}

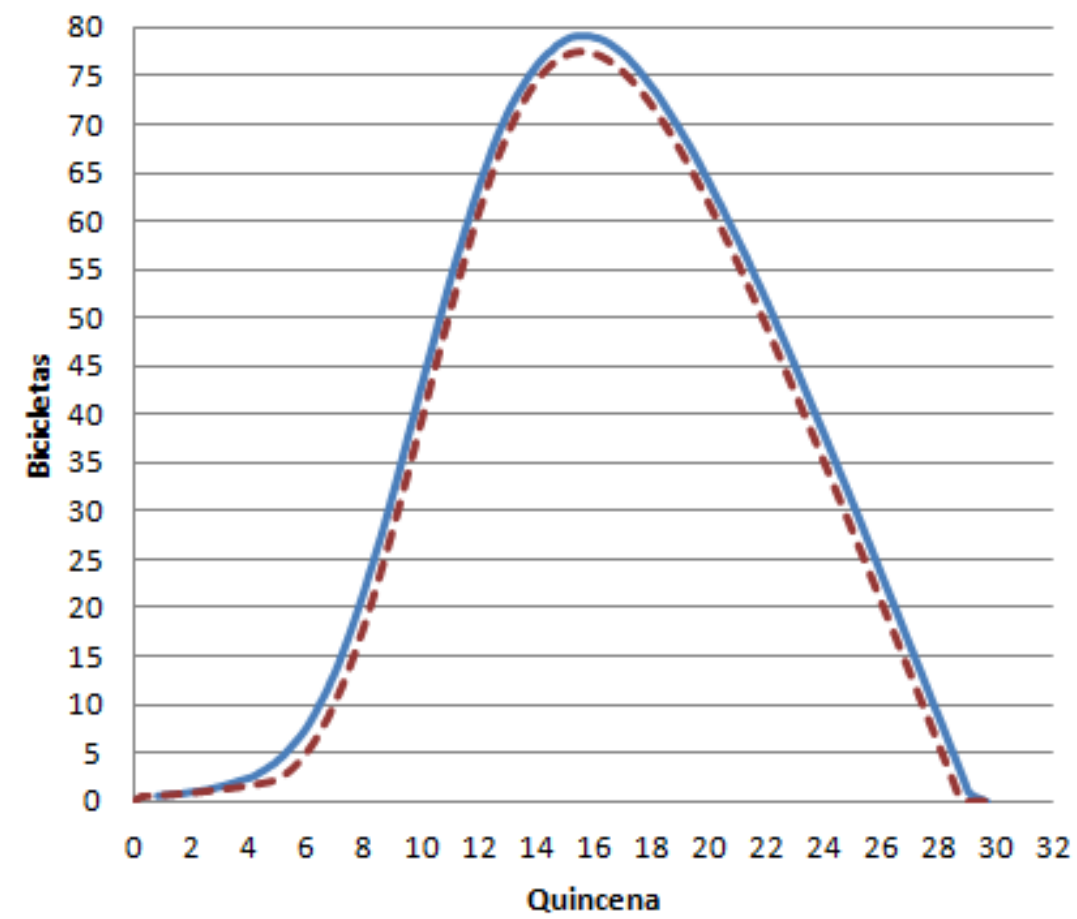

DES: Promedio - - - SD

Elaboración propia.

¿Qué hubiera sucedido si se hubiera dado prioridad en la fabricación a las órdenes de producción del modelo STEALTH?

Solo se puede dar respuesta a esta pregunta con el modelo de DES, que al nivel de entidades permite cambiar la secuencia de procesamiento. En ARENA esto se hace desde el módulo QUEUE. Si por ejemplo, cuando llega una orden del modelo STEALTH hay en espera en ese momento 5 órdenes del modelo VELOZ, la del modelo STEALTH lleva la prioridad y se coloca delante de las otras 5 órdenes.

Con esta nueva prioridad, el valor promedio del TPOP obtenido para el modelo VELOZ fue de 8.8 quincenas o 132 días. La mitad de la longitud del intervalo de confianza para dicho valor fue $<0.02$. Además, el valor promedio del TPOP para el modelo STEALTH fue de 0.95 quincenas o 14.3 días. La mitad de la longitud del intervalo de confianza para dicho valor fue $<0.09$. 
Claramente en este caso hay una mayor espera de las órdenes del modelo VELOZ que con la regla FIFO. A continuación se muestra en la Figura 3.35 la curva de las Ordenes de Producción del modelo DES. Hay una diferencia bastante grande con la curva obtenida con el modelo SD (Figura 3.34) cuyo paradigma asume a las entidades moviéndose como un flujo continuo homogéneo y que sólo pudo ser representado con un modelo PROMEDIO.

Figura 3.35.

Comparación SD y DES: Ordenes de Producción-prioridad para STEALTH.
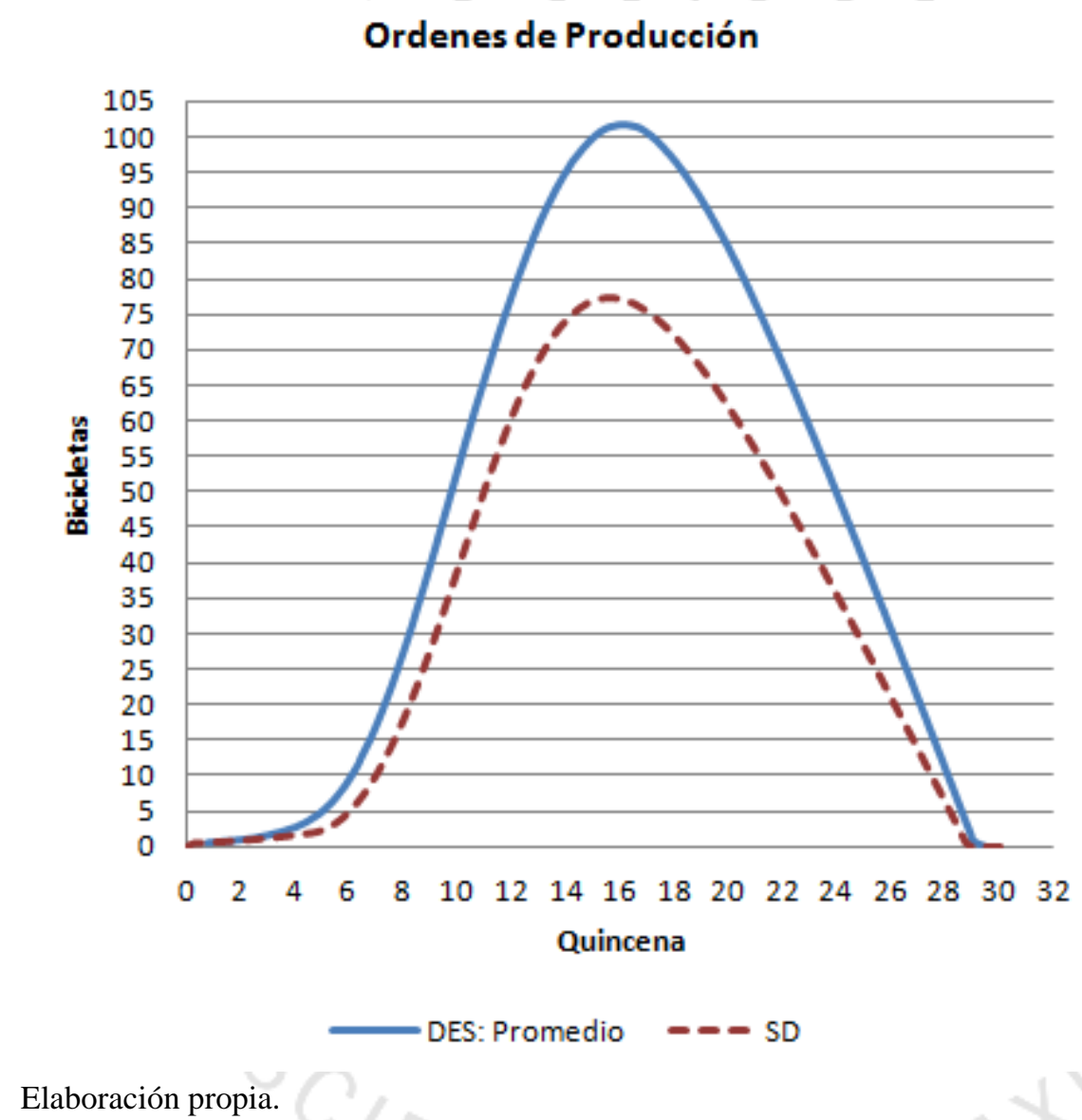

En el modelo DES las órdenes acumuladas alcanzan su valor máximo de 101.8 órdenes en la quincena 16. Lo cual ya muestra una diferencia con las 77.5 órdenes que arroja el modelo SD, el cual con ese modelo PROMEDIO no puede reflejar el efecto de la priorización. Y es debido a esta priorización que las órdenes de producción del modelo STEALTH generan una acumulación de órdenes mucho mayor a la mostrada por la regla FIFO y con tiempos de procesamiento más altos para el modelo VELOZ que es el más vendido de ESR. Debido a ello, la gerencia debe recapacitar si vale la 
pena implementar esta regla antes de decidir en aumentar el número de líneas de producción.

\subsubsection{Escenario 5: Decisiones heterogéneas de compra}

ESR decide contratar a una compañía de mercadeo para haga un estudio que profundice el conocimiento sobre las intenciones de compra de las personas de la población objetivo. Este estudio determina que la decisión de adquisición del producto por el boca a boca no está asociada con una probabilidad homogénea de compra (valor de 0.045 usado hasta ahora) como se había determinado en el escenario original. Por el contrario, determina que cuando se encuentran dos personas, una que ya ha adquirido el producto y la otra que no lo tiene, la influencia o efectividad (entusiasmo por el producto) que tiene la primera persona para que la segunda persona compre el producto depende del tiempo transcurrido desde que la primera persona adquirió el producto. Este comportamiento se muestra en la Figura 3.36 y se procede a dar su explicación.

Figura 3.36.

Probabilidad variable de adquisición del producto.

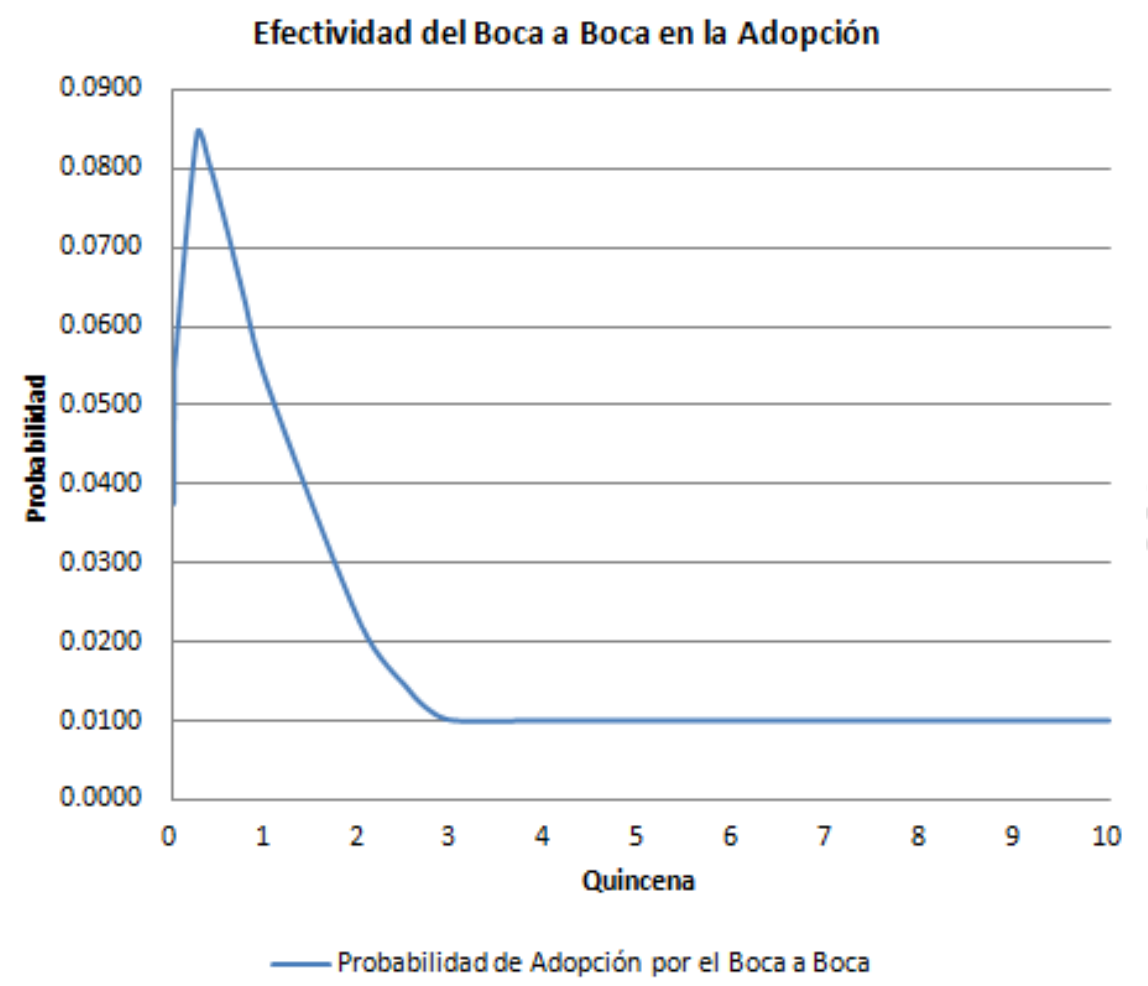

Elaboración propia.

Durante los primeros días de adquisición del producto existe una gran emoción por la experiencia de descubrir los beneficios del nuevo producto y que se transmite en 
una gran poder de convencimiento e influencia sobre las otras personas para que también compren el producto. Luego esa emoción va disminuyendo con el paso del tiempo. La compañía de mercadeo propuso la siguiente curva de probabilidades de adquisición del producto para una persona que no lo tiene, en base al tiempo que ha transcurrido desde que la persona que busca convencerla compró el producto. Esta curva se muestra en la Figura 3.36. La probabilidad es variable hasta la tercera quincena y luego a de ahí en adelante se vuelve constante.

En este escenario, la probabilidad de compra del producto va a depender de la iteración entre dos personas para representar la influencia de una sobre la otra, por lo tanto, esta situación es ideal para usar ABS. No se puede modelar este caso usando SD o DES porque no se llega al detalle de representar dichas iteraciones en estos paradigmas.

Usando el modelo de ABS, luego de unas corridas iniciales, se determinó que el tiempo requerido para cubrir el mercado potencial era de 52 quincenas. El doble de tiempo que en el escenario original. Se hicieron 50 replicaciones de 41600 iteraciones cada una. Estas 41600 iteraciones representan a 52 quincenas. Sin embargo, como se aprecia en la Figura 3.37, el mercado potencial realmente ha sido prácticamente cubierto en las primeras 40 quincenas.

Figura 3.37.

Mercado Potencial y Compradores para el Escenario 5.

\section{Compradores y Mercado Potencial}

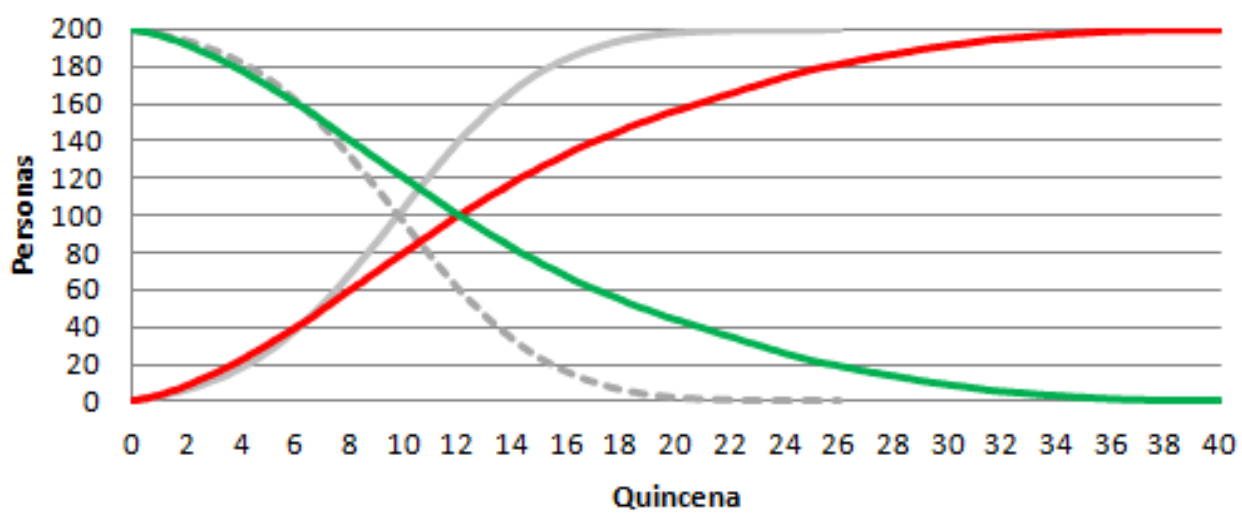

- ABS (Esc. Original): Promedio Compradores

- - ABS (Esc. Original): Promedio Mercado Potencial

ABS (Esc. 5): Promedio Compradores

ABS (Esc. 5): Promedio Mercado Potencial

Elaboración propia. 
El hecho de mostrar sólo las primeras 40 quincenas se debe a una limitación de Excel solo permite graficar 32000 puntos (donde cada punto está asociado a una iteración del modelo de ABS). Estas 32000 iteraciones representan las 40 quincenas. Sin embargo, la fracción del mercado potencial correspondiente a las restantes 12 quincenas (52-40) es tan ínfima que prácticamente es suficiente mostrar las primeras 40 quincenas.

Cuando se compara con las curvas de Compradores y Mercado potencial generadas con ABS en el escenario original, se puede apreciar que el tiempo de intersección (se cubrió el 50\% del mercado) hallado con ABS de este escenario fue de 12.04 quincenas y difiere con el punto hallado en el escenario original con ABS de 9.78 quincenas. Además, el tiempo requerido para cubrir el 50\% restante del mercado potencial si es bastante mayor en este escenario (27.96 quincenas contra las 16.22 quincenas del escenario original). Esto se debe a que la probabilidad de adopción en este escenario para las 100 personas restantes del mercado potencial es igual a 0.01 (ver Figura 3.36) mientras que en el escenario original se mantiene en su valor constante de 0.045 .

A continuación se presenta en la Figura 3.38 la evolución de las órdenes de producción a lo largo del tiempo.

Figura 3.38.

Ordenes de Producción para el Escenario 5.

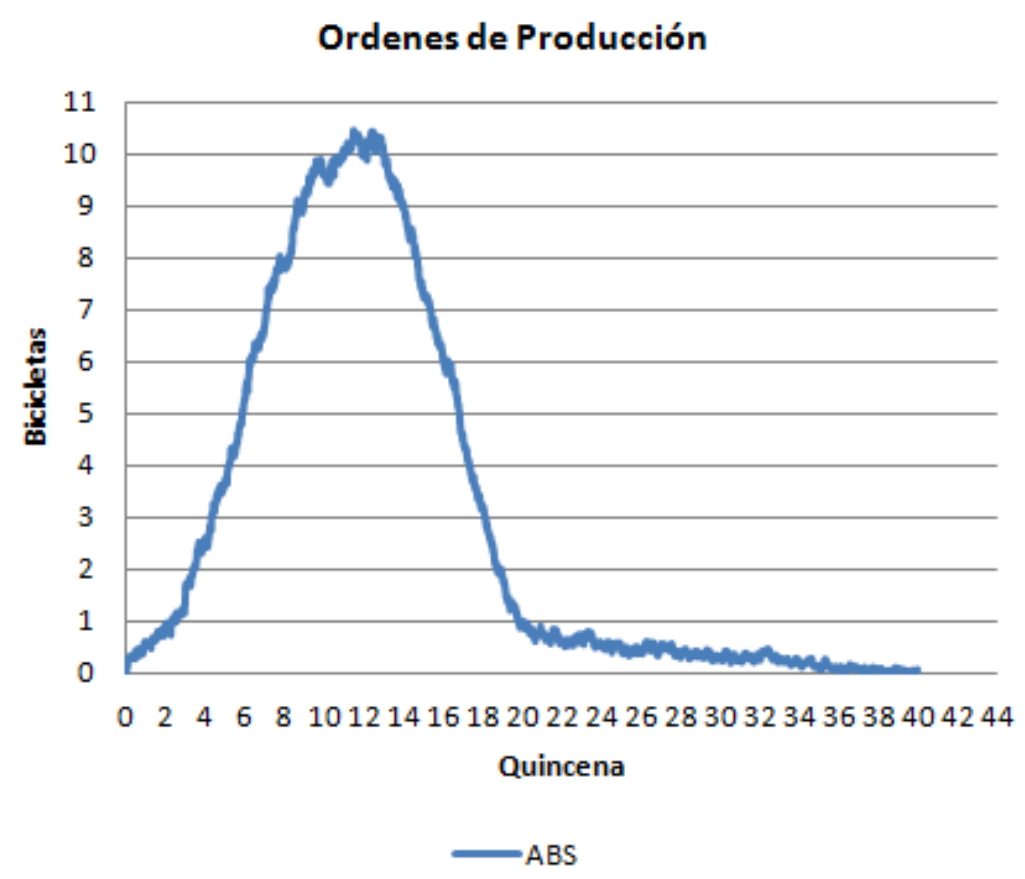

Elaboración propia. 
Al ser más espaciado el proceso de compras en este escenario que en el escenario original no se produce la acumulación tan alta de órdenes de producción. El nivel máximo de 10.48 órdenes se alcanza en la quincena 11.49 .

Sin embargo, se puede apreciar que la curva promedio no es una curva suave sino que muestra varios picos. Esto se debe a la variabilidad de las probabilidades de adquisición del producto por el boca a boca que son bastante distintas de una replicación a otra.

\subsubsection{Escenario 6: Redes sociales}

En este escenario se considera que las 200 personas que conforman el mercado potencial para la empresa San Remo están interconectadas a través de redes sociales donde cada persona sólo interactúa con las otras personas con las que está relacionada. Esto hace una diferencia con el escenario original donde cada una de las personas de la población objetivo podía relacionarse con cualquiera de las otras personas de la misma población objetivo. A continuación se muestra en la Figura 3.39 el ejemplo de una estructura de red social donde cada punto azul representa a una de estas personas.

Figura 3.39.

Conexiones de las redes sociales.

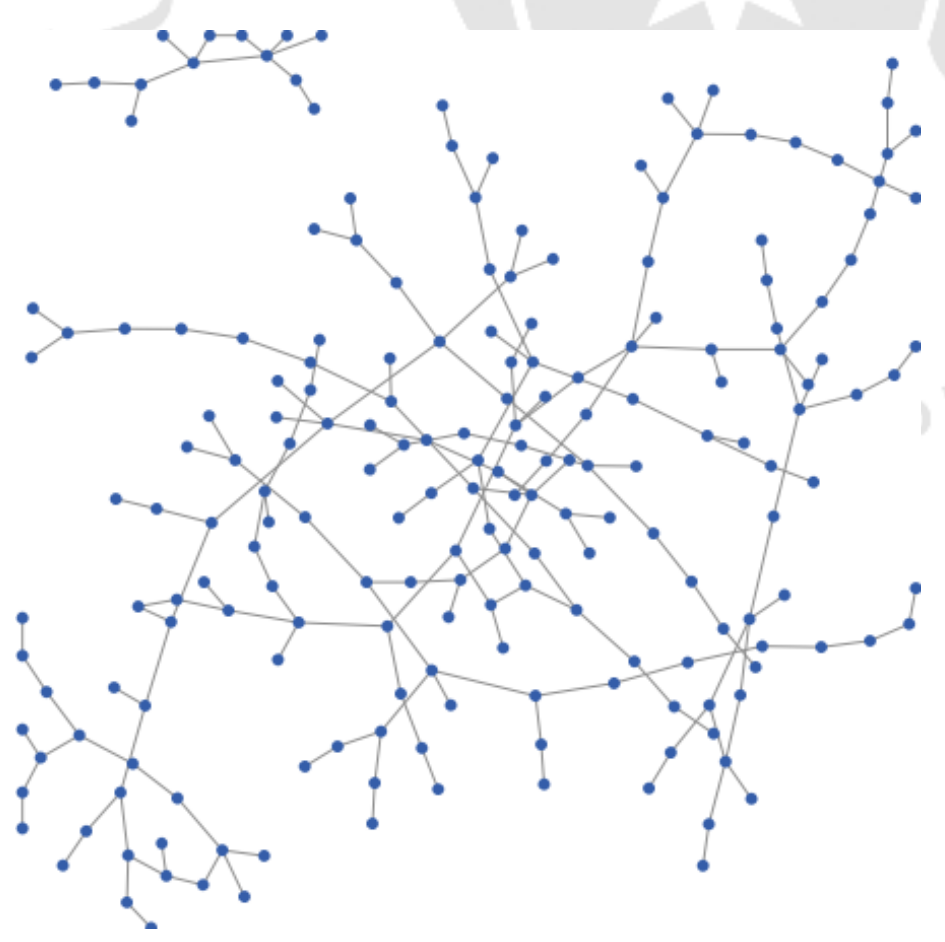

Elaboración propia. 
Este tipo de iteraciones con interconexiones muy particulares para cada persona no se pueden modelar con SD o DES. Para ello se usó un modelo de ABS donde la estructura de estas redes fue generada de forma aleatoria. Es decir, en cada replicación de la simulación del modelo de ABS se tendrá una estructura distinta y por lo tanto el número de conexiones variará de una persona a otra. De la figura anterior se puede apreciar que pueden generarse grupos aislados de personas con respecto a la estructura mayor de la red. En estos casos, la adopción por el boca a boca recién se empezará a dar cuando la primera persona miembro de ese grupo adopte el producto por efecto de la publicidad.

En este escenario se siguieron todos los pasos descritos en la Figura 3.19 para el escenario original. La única diferencia es en el Paso 5 donde las segunda persona elegida no es cualquier persona de la población objetivo sino una persona que está relacionada por la red social con la primera persona seleccionada en el mismo paso. Por otro lado, de forma similar a como se hizo en el escenario 5, en este escenario se realizaron 50 replicaciones de 41600 iteraciones cada una. Estas 41600 iteraciones representan a 52 quincenas, sin embargo, como se aprecia en la Figura 3.40, el mercado potencial realmente ha sido cubierto prácticamente en las primeras 40 quincenas.

Figura 3.40.

Mercado Potencial y Compradores para el Escenario 6.

\section{Compradores y Mercado Potencial}

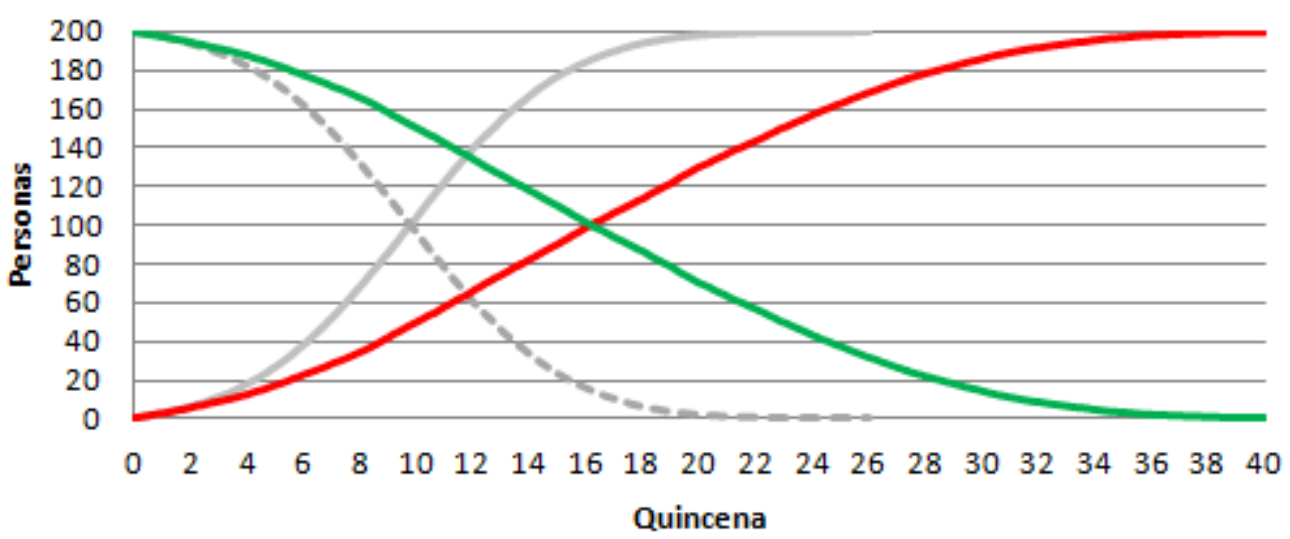

- ABS (Esc. Original): Promedio Compradores

- - ABS (Esc. Original): Promedio Mercado Potencial

ABS (Esc. 6): Promedio Compradores

ABS (Esc. 6): Promedio Mercado Potencial

Elaboración propia. 
Cuando más interconectadas se encuentran las personas la difusión del producto por el boca a boca se realiza más rápidamente. Por lo tanto, como en este escenario las personas de la población objetivo se encuentran menos interconectadas que en el escenario original, se espera un proceso de compra del producto más lento que el del escenario original. En este escenario el tiempo de intersección para alcanzar el 50\% del mercado potencial es de 16.06 quincenas contra las 9.78 quincenas del modelo de ABS del escenario original.

Este proceso más extendido de la difusión de las compras del producto permite que no se formen grandes colas de las órdenes de producción en espera de ser procesadas en las dos líneas de fabricación como se muestra en la Figura 3.41.

Figura 3.41.

Ordenes de Producción para el Escenario 6.

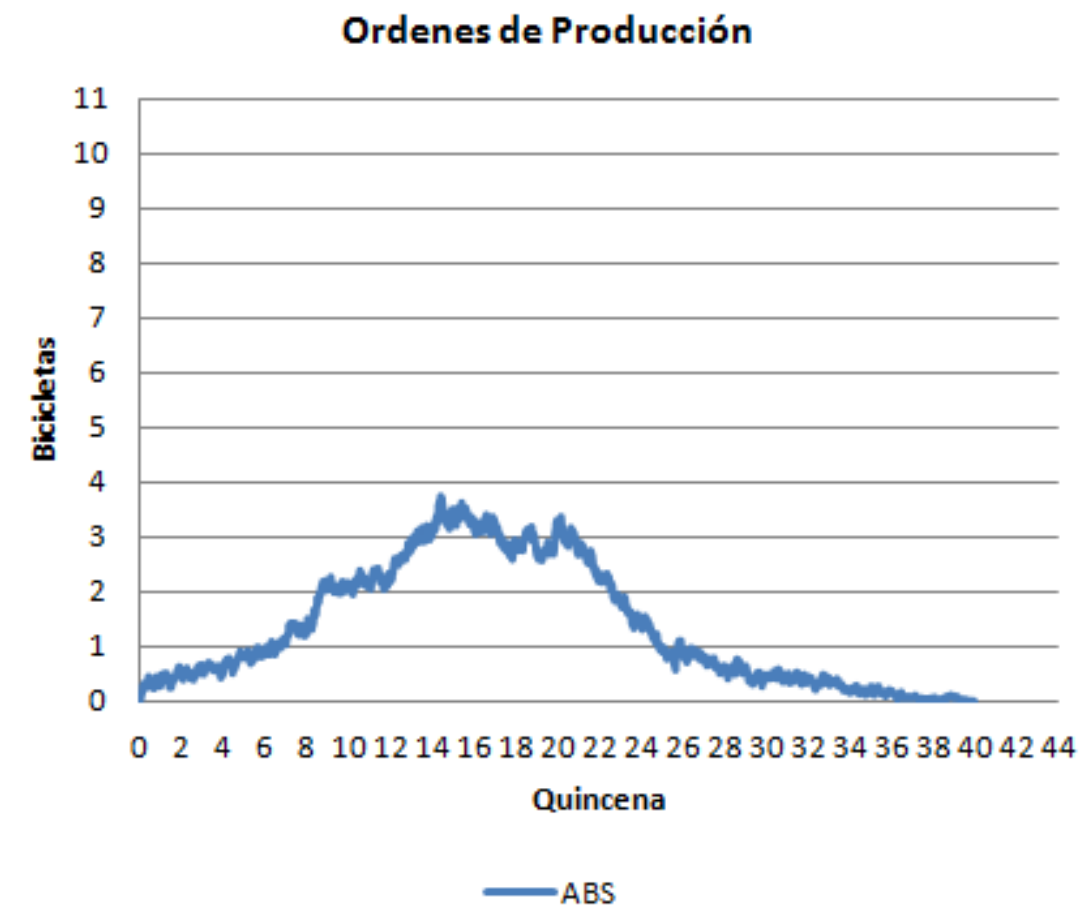

Elaboración propia. 


\subsubsection{Resumen comparativo de los escenarios}

A continuación se muestra un resumen de las conclusiones obtenidas en el análisis de los escenarios por cada uno de los paradigmas.

Tabla 3.4

Desempeño de los paradigmas por escenario analizado.

\begin{tabular}{|c|c|c|c|}
\hline Esce & SD) & $\begin{array}{l}\text { Simulación de Eventos } \\
\text { Discretos (DES) }\end{array}$ & tes \\
\hline $\begin{array}{l}\text { Escenario 1. } \\
\text { Objetivo: } \\
\text { Representar curvas de } \\
\text { evolución del mercado y } \\
\text { recolectar estadísticas } \\
\text { promedio del proceso de } \\
\text { fabricación: } \\
\text { - TPOP } \\
\text { - Tiempo en cola } \\
\text { - Utilización de los } \\
\text { recursos } \\
\text { Características: } \\
\text { - Entidades homogéneas } \\
\text { (un sólo modelo de } \\
\text { bicicleta:VELOZ) } \\
\text { - Tiempos aleatorios } \\
\text { - Interrelación entre } \\
\text { Consumidores y }\end{array}$ & $\begin{array}{l}\text { Ventajas } \\
\text { - } \quad \text { Al ser las entidades } \\
\text { homogéneas se pueden tomar } \\
\text { estadísticas agregadas en una } \\
\text { sola corrida al trabajar con } \\
\text { valores promedio. } \\
\text { - Generación de los gráficos de } \\
\text { las curvas es rápida al } \\
\text { obtenerse los valores de la } \\
\text { variables directamente del } \\
\text { reporte de resultados. } \\
\text { Desventajas } \\
\text { Estadísticas no salen } \\
\text { directamente del reporte de } \\
\text { resultados hay que } \\
\text { construirlas (agregando al } \\
\text { modelo variables de nivel y } \\
\text { flujo) } \\
\text { No permite recolectar }\end{array}$ & $\begin{array}{l}\text { Ventajas } \\
\text { - Recolección de estadísticas es } \\
\text { en forma directa del reporte } \\
\text { de resultados. Se deben hacer } \\
\text { modificaciones sencillas al } \\
\text { modelo original. } \\
\text { Desventajas } \\
\text { - Se requieren múltiples } \\
\text { replicaciones y generar } \\
\text { intervalos de confianza. }\end{array}$ & $\begin{array}{l}\text { Desventajas } \\
\text { - Al no requerirse un } \\
\text { modelamiento de } \\
\text { interrelaciones heterogéneas } \\
\text { entre los agentes (entidades) } \\
\text { entonces no justifica el } \\
\text { esfuerzo de representar y } \\
\text { calcular los efectos de dichas } \\
\text { interacciones en detalle. } \\
\text { Las transacciones que se requiere } \\
\text { procesar con DES en una } \\
\text { replicación es de } 200 \text { entidades. } \\
\text { Mientras que las transacciones } \\
\text { que se requiere procesar con ABS } \\
\text { en una replicación es de } 20800 \\
\text { interacciones entre agentes. } \\
\text { Por lo tanto en este escenario es } \\
\text { preferible usar DES en vez de }\end{array}$ \\
\hline
\end{tabular}




\begin{tabular}{|c|c|c|c|}
\hline Escenario & Dinámica de Sistemas (SD) & \begin{tabular}{|l} 
Simulación de Eventos \\
Discretos (DES)
\end{tabular} & $\begin{array}{l}\text { Simulación Basada en Agentes } \\
\text { (ABS) }\end{array}$ \\
\hline $\begin{array}{l}\text { Consumidores Potenciales } \\
\text { es homogénea, } \\
\text { permitiendo trabajar con } \\
\text { tasas de adopción } \\
\text { promedio }\end{array}$ & $\begin{array}{l}\text { estadísticas a nivel de entidad. } \\
\text { Por ejemplo, tiempo } \\
\text { promedio de espera en cola de } \\
\text { cada orden de producción. }\end{array}$ & & $\begin{array}{l}\text { ABS. Los resultados producidos } \\
\text { serían los mismos pero de una } \\
\text { manera más sencilla al } \\
\text { representar sólo los efectos } \\
\text { promedio de las interrelaciones. }\end{array}$ \\
\hline $\begin{array}{l}\text { Escenario } 2 . \\
\text { Objetivo: } \\
\text { Hacer un análisis de las } \\
\text { ventas: } \\
\text { - Identificar causales del } \\
\quad \text { comportamiento de la } \\
\text { curva de ventas } \\
\text { - Obtención de } \\
\text { probabilidades de } \\
\text { penetración de mercado } \\
\text { Características: } \\
\text { Se mantienen las mismas } \\
\text { características del escenario } 1\end{array}$ & $\begin{array}{l}\text { Ventajas } \\
\text { - } \quad \text { Existen metodologías para } \\
\text { hacer análisis de tipo causal e } \\
\text { identificar los bucles } \\
\text { dominantes responsables por } \\
\text { el comportamiento de las } \\
\text { curvas de variables como las } \\
\text { ventas. Por ejemplo, el efecto } \\
\text { de la publicidad sólo } \\
\text { contribuye de forma inicial al } \\
\text { comportamiento de la curva } \\
\text { ventas y luego quien tiene } \\
\text { mayor impacto sobre las } \\
\text { ventas es el efecto del boca a } \\
\text { boca. } \\
\text { Desventajas } \\
\text { Al manejar tiempos promedio } \\
\text { no se puede hacer un análisis } \\
\text { de tipo probabilístico }\end{array}$ & \begin{tabular}{|l} 
Ventajas \\
- Al trabajar con variables \\
aleatorias se puede incorporar \\
fácilmente el análisis de tipo \\
probabilístico. Como por \\
ejemplo, calcular la \\
probabilidad de alcanzar el \\
$50 \%$ del mercado en menos \\
de 10.1 quincenas. \\
Desventajas \\
El análisis de regresión lineal \\
no implica causalidad sino \\
correlación. Además otro \\
problema es que tampoco se \\
pudo identificar correlaciones \\
de tipo no lineal entre la \\
variables, tales como las que \\
ocurren en el modelo de Bass.
\end{tabular} & $\begin{array}{l}\text { Desventajas } \\
\text { - } \quad \text { ABS puede usar las mismas } \\
\text { metodologías de análisis } \\
\text { probabilístico que DES, pero } \\
\text { se prefiere usar DES en este } \\
\text { caso, al sólo requerirse la } \\
\text { representación de los efectos } \\
\text { promedios de las } \\
\text { interrelaciones entre las } \\
\text { personas. }\end{array}$ \\
\hline Escenario 3 & $\begin{array}{l}\text { Ventajas } \\
\text { - Tiempo de procesamiento }\end{array}$ & $\begin{array}{l}\text { Desventajas } \\
\text { - Tiempo de procesamiento }\end{array}$ & $\begin{array}{l}\text { Desventajas } \\
\text { - Se requiere mucho más }\end{array}$ \\
\hline
\end{tabular}




\begin{tabular}{|c|c|c|c|}
\hline Escenario & Dinámica de Sistemas (SD) & $\begin{array}{l}\text { Simulación de Eventos } \\
\text { Discretos (DES) }\end{array}$ & $\begin{array}{l}\text { Simulación Basada en Agentes } \\
\text { (ABS) }\end{array}$ \\
\hline $\begin{array}{l}\text { Objetivo: } \\
\text { Representar curvas de } \\
\text { evolución del mercado y } \\
\text { recolectar estadísticas } \\
\text { promedio del proceso de } \\
\text { fabricación ante una } \\
\frac{\text { expansión del mercado de }}{200 \text { a } 10 \text { mil personas: }} \\
\text { - Número máximo de } \\
\quad \text { órdenes en cola } \\
\text { - Utilización de los } \\
\text { recursos } \\
\text { - Punto de inflexión } \\
\text { - Punto de intersección } \\
\text { Características: } \\
\text { Se mantienen las mismas } \\
\text { características del escenario } 1\end{array}$ & $\begin{array}{l}\text { computacional es rápido. No } \\
\text { varía mucho con respecto al } \\
\text { tamaño del mercado porque } \\
\text { no se consideran entidades } \\
\text { individuales. }\end{array}$ & $\begin{array}{l}\text { computacional puede llegar a } \\
\text { ser lento en modelos grandes. } \\
\text { En cada replicación se deben } \\
\text { analizar } 10 \text { mil entidades. } \\
\text { - Generación de gráficos de las } \\
\text { curvas requiere más tiempo de } \\
\text { procesamiento previo de la } \\
\text { información (replicaciones) } \\
\text { en otra aplicación como } \\
\text { EXCEL. }\end{array}$ & $\begin{array}{l}\text { tiempo de procesamiento que } \\
\text { en DES y por lo tanto } \\
\text { tampoco puede competir con } \\
\text { SD en este escenario. }\end{array}$ \\
\hline $\begin{array}{l}\text { Escenario } 4 \\
\text { Objetivo: } \\
\text { Representar curvas de } \\
\text { evolución del mercado y } \\
\text { recolectar estadísticas } \\
\text { promedio del proceso de } \\
\text { fabricación para dos modelos } \\
\text { de bicicletas: } \\
\text { - TPOP }\end{array}$ & $\begin{array}{l}\text { Ventajas } \\
\text { - Los dos modelos de bicicletas } \\
\text { se representan como un sólo } \\
\text { modelo promedio de } \\
\text { bicicleta, permitiendo tomar } \\
\text { estadísticas agregadas en una } \\
\text { sola corrida al trabajar con un } \\
\text { tiempo promedio de } \\
\text { producción asociado con ese } \\
\text { modelo único. }\end{array}$ & $\begin{array}{l}\text { Ventajas } \\
\text { - Recolección de estadísticas es } \\
\text { en forma directa del reporte } \\
\text { de resultados. Se deben hacer } \\
\text { modificaciones sencillas al } \\
\text { modelo original. Por ejemplo, } \\
\text { definir un atributo que } \\
\text { identifique el modelo de } \\
\text { - } \text { bicicleta. } \\
\text { - Al tratar cada modelo como }\end{array}$ & $\begin{array}{l}\text { Desventajas } \\
\text { - Se requiere mucho más } \\
\text { tiempo de procesamiento para } \\
\text { obtener los mismos resultados } \\
\text { que en DES y por lo tanto en } \\
\text { este caso se debe usar DES en } \\
\text { lugar de ABS. }\end{array}$ \\
\hline
\end{tabular}




\begin{tabular}{|c|c|c|c|}
\hline Escenario & Dinámica de Sistemas (SD) & $\begin{array}{l}\text { Simulación de Eventos } \\
\text { Discretos (DES) }\end{array}$ & $\begin{array}{l}\text { Simulación Basada en Agentes } \\
\text { (ABS) }\end{array}$ \\
\hline 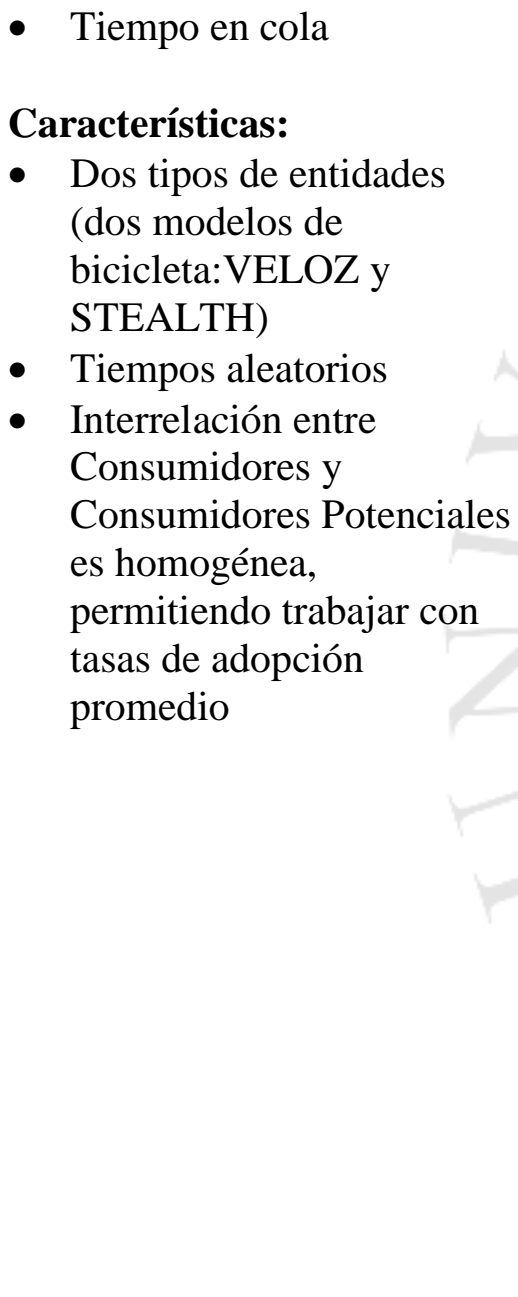 & 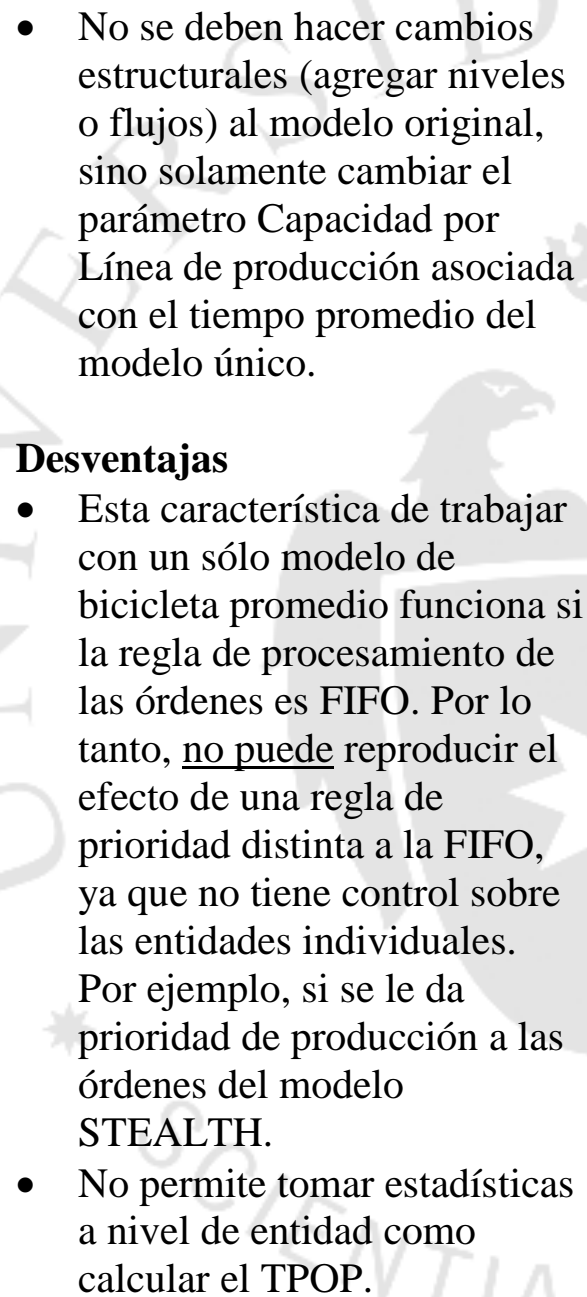 & $\begin{array}{l}\text { una entidad individual se } \\
\text { puede definir prácticamente } \\
\text { cualquier regla de } \\
\text { procesamiento de las órdenes } \\
\text { de producción. }\end{array}$ & 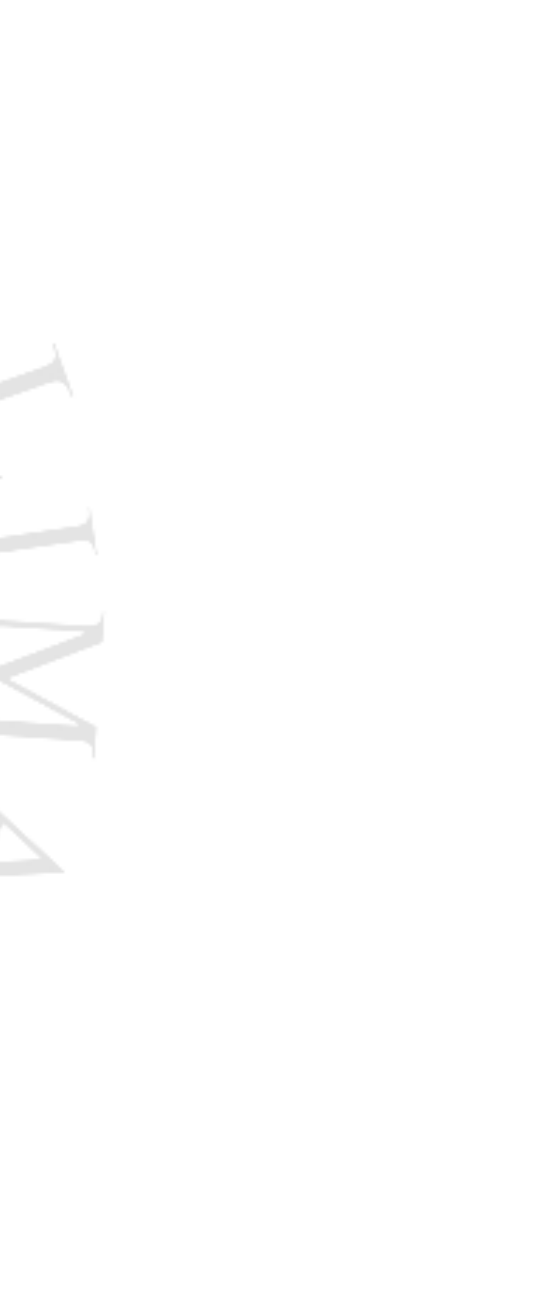 \\
\hline Escenario 5 & Desventajas & Desventajas & Ventajas \\
\hline
\end{tabular}




\begin{tabular}{|c|c|c|c|}
\hline Escenario & Dinámica de Sistemas (SD) & $\begin{array}{l}\text { Simulación de Eventos } \\
\text { Discretos (DES) } \\
\end{array}$ & $\begin{array}{l}\text { Simulación Basada en Agentes } \\
\text { (ABS) }\end{array}$ \\
\hline $\begin{array}{l}\text { Objetivo: } \\
\text { Representar curvas de } \\
\text { evolución del mercado y } \\
\text { recolectar estadísticas } \\
\text { promedio con decisiones de } \\
\text { compra heterogéneas: } \\
\text { - Punto de intersección } \\
\text { - Número máximo de } \\
\text { órdenes en cola } \\
\text { Características: } \\
\text { - Entidades homogéneas } \\
\text { (un sólo modelo de } \\
\text { bicicleta:VELOZ) } \\
\text { - Tiempos aleatorios } \\
\text { - Interrelación entre } \\
\text { Consumidores y } \\
\text { Consumidores Potenciales } \\
\text { no es homogénea. Sino } \\
\text { que la decisión de compra } \\
\text { del Consumidor Potencial } \\
\text { depende del la influencia } \\
\text { o efectividad (entusiasmo } \\
\text { por el producto) asociada } \\
\text { por el tiempo transcurrido } \\
\text { desde que el Consumidor } \\
\text { adquirió el producto. }\end{array}$ & $\begin{array}{l}\text { - SD simplemente no es capaz } \\
\text { de representar un } \\
\text { modelamiento detallado de } \\
\text { las interacciones entre las } \\
\text { entidades que se requiere en } \\
\text { este escenario. Es decir, en } \\
\text { este caso no se puede trabajar } \\
\text { con representaciones } \\
\text { promedio de las tasas de } \\
\text { adopción. }\end{array}$ & $\begin{array}{l}\text { DES (de forma natural) no es } \\
\text { capaz de representar un } \\
\text { modelamiento detallado de las } \\
\text { interacciones entre las } \\
\text { entidades que se requiere en } \\
\text { este escenario. }\end{array}$ & $\begin{array}{l}\text { - Este es el caso ideal para } \\
\text { ABS, ya que permite generar } \\
\text { el comportamiento global de } \\
\text { las compras que emerge de las } \\
\text { interrelaciones de compras } \\
\text { individuales entre los agentes. } \\
\text { Desventajas } \\
\text { - Tiempo de procesamiento } \\
\text { computacional puede llegar a } \\
\text { ser lento en modelos grandes. } \\
\text { En cada replicación se deben } \\
\text { analizar } 40600 \text { iteraciones. } \\
\text { Generación de gráficos de las } \\
\text { curvas requiere mucho tiempo } \\
\text { de procesamiento previo de la } \\
\text { información (replicaciones) } \\
\text { en otra aplicación como } \\
\text { EXCEL. }\end{array}$ \\
\hline
\end{tabular}




\begin{tabular}{|c|c|c|c|}
\hline Escenario & Dinámica de Sistemas (SD) & $\begin{array}{l}\text { Simulación de Eventos } \\
\text { Discretos (DES) }\end{array}$ & $\begin{array}{l}\text { Simulación Basada en Agentes } \\
\text { (ABS) }\end{array}$ \\
\hline $\begin{array}{l}\text { Escenario } 6 \\
\text { Objetivo: } \\
\text { Representar curvas de } \\
\text { evolución del mercado y } \\
\text { recolectar estadísticas } \\
\text { promedio en un entorno de } \\
\text { redes sociales: } \\
\text { - Punto de intersección } \\
\text { - Número máximo de } \\
\text { órdenes en cola } \\
\text { Características: } \\
\text { - Entidades homogéneas } \\
\text { (un sólo modelo de } \\
\text { bicicleta:VELOZ) } \\
\text { - Tiempos aleatorios } \\
\text { Interrelación entre } \\
\text { Consumidores y } \\
\text { Consumidores Potenciales } \\
\text { sólo ocurre entre las } \\
\text { personas que están } \\
\text { interconectadas a través } \\
\text { de la red social. }\end{array}$ & $\begin{array}{l}\text { Desventajas } \\
\text { - SD simplemente no es capaz } \\
\text { de representar la red social } \\
\text { que se detalla a nivel de } \\
\text { entidades. }\end{array}$ & $\begin{array}{l}\text { Desventajas } \\
\text { - DES no es capaz de } \\
\text { representar la red social que } \\
\text { involucra una interconexión } \\
\text { entre las entidades. }\end{array}$ & $\begin{array}{l}\text { Ventajas } \\
\text { - } \quad \text { Este es el caso ideal para } \\
\text { ABS, ya que permite } \\
\text { representar de forma sencilla } \\
\text { las interrelaciones entre los } \\
\text { agentes que conforman la red } \\
\text { social. } \\
\text { Desventajas } \\
\text { - Tiempo de procesamiento } \\
\text { computacional puede llegar a } \\
\text { ser lento en modelos grandes. } \\
\text { En cada replicación se deben } \\
\text { analizar 40600 iteraciones. } \\
\text { Generación de gráficos de las } \\
\text { curvas requiere mucho tiempo } \\
\text { de procesamiento previo de la } \\
\text { información (replicaciones) } \\
\text { en otra aplicación como } \\
\text { EXCEL. }\end{array}$ \\
\hline
\end{tabular}

Elaboración propia. 


\section{CONCLUSIONES}

En este trabajo de investigación se identificaron y compararon las características cualitativas y cuantitativas de los tres paradigmas de la simulación: dinámica de sistemas, simulación de eventos discretos y simulación basada en agentes.

La dinámica de sistemas es un método utilizado para representar la estructura de sistemas complejos y entender su comportamiento a través del tiempo. Matemáticamente un modelo de dinámica de sistemas es un modelo de simulación continua representada por un conjunto de ecuaciones diferenciales. La idea esencial en dinámica de sistemas es que todos los elementos en un sistema interactúan a través de relaciones causales y donde la estructura del sistema determina su comportamiento dinámico. La estructura del sistema se modela con flujos y bucles de realimentación conectados a niveles. Esta técnica se usa generalmente para describir los sistemas del mundo real con una visión macroscópica de arriba hacia abajo a través de relaciones no lineales entre entidades consideradas homogéneas y representadas de forma agregada.

La dinámica de sistemas es ideal para situaciones en las que el comportamiento de un gran número de entidades homogéneas se puede representar de forma agregada y en base a valores promedio. Por ejemplo, un mercado de 10 mil personas donde no es necesario representar a cada una de ellas de forma individual. Otra situación ventajosa para usar dinámica de sistemas es que permite representar relaciones causales donde los procesos de retroalimentación juegan un papel preponderante; contando con métodos de análisis para identificar los bucles dominantes responsables por el comportamiento dinámico del sistema. Esta técnica no es adecuada cuando se requiere modelar a entidades heterogéneas de forma individual, lo cual no permite recolectar estadísticas como por ejemplo el tiempo promedio en cola de una entidad.

La simulación de eventos discretos representa el mundo como entidades que fluyen a través de una red de colas y actividades, en donde los recursos son compartidos entre las actividades. Es muy útil para problemas que consisten en simulaciones de colas o redes complejas con colas, en las cuales los procesos pueden ser bien definidos y el énfasis está en representar incertidumbre a través de distribuciones estocásticas. Pertenece a la clasificación de simulación discreta, donde el estado del sistema sólo puede cambiar en puntos discretos de tiempo en los que ocurren eventos. Los modelos 
son típicamente de bucle abierto (open-loop) donde la retroalimentación no juega un rol preponderante. Permite representar las entidades a nivel individual y en forma heterogénea. Las entidades se consideran objetos pasivos donde la secuencia de ellas dentro del modelo está dominada por reglas globales preestablecidas. Se requiere el uso de métodos estadísticos para el análisis de los datos de entrada y los resultados.

La simulación de eventos discretos es adecuada para representar de forma detallada a entidades heterogéneas y múltiples recursos en situaciones donde los efectos estocásticos son importantes y no se pueden plasmar simplemente con valores promedio. Además, permite incorporar análisis de tipo probabilístico de los resultados. Por otro lado, este paradigma de simulación no puede representar (de forma natural) situaciones donde el comportamiento del sistema se origina de las interrelaciones entre las entidades ya que éstas son por defecto de tipo pasivo.

La simulación basada en agentes es un método de abajo hacia arriba, es decir, la interacción entre los agentes determina el comportamiento del sistema. El componente básico usado en la construcción de un modelo es el agente y se les considera entidades "activas" ya que pueden tomar decisiones autónomas no preestablecidas, es decir, pueden tomar decisiones sin un controlador central. Para lograr esto, tienen un conjunto de reglas que determina su comportamiento. Estas reglas pueden variar desde simples formas determinísticas del tipo "si-entonces" o en reglas basadas en probabilidades. En este tipo de simulación existen dos métodos para avanzar el reloj: el método de avance de tiempo de incremento fijo (ó actualización asincrónica) y el método de avance del siguiente evento (ó actualización sincrónica).

La simulación basada en agentes permite representar las interrelaciones entre entidades heterogéneas (agentes) y las conexiones dinámicas entre ellas. Es el paradigma adecuado de utilizar cuando no se conocen las reglas globales que rigen el sistema y éstas deben emerger del comportamiento individual. Justamente, cuando no se requiere que las entidades tengan este nivel autónomo de decisión y no se necesita generar las interrelaciones entre ellas se debe usar la simulación de eventos discretos, porque la desventaja de la simulación basada en agentes es el extenso tiempo de procesamiento computacional que se toma al trabajar a ese nivel de detalle. 
Finalmente, teniendo en cuenta la visión o enfoque de cada paradigma y las características del problema que se quiere representar se debe elegir el método de simulación adecuado para construir el modelo correspondiente. 


\section{RECOMENDACIONES PARA TRABAJO FUTURO}

Frecuentemente un problema no puede ser enfocado exclusivamente con un sólo paradigma de simulación. Usando una metodología basada en un sola técnica de simulación puede traer como consecuencia que en muchos casos el modelador termine haciendo uso de construcciones no naturales e incómodas para representar la realidad o simplemente deje esa parte complicada del problema como fuera del alcance del modelo tratándolo como exógeno (Borshchev, 2013). La tendencia es modelar haciendo uso de múltiples paradigmas en forma simultánea. Esto hace que el proceso de modelamiento sea más sencillo e incrementa su flexibilidad. Para ello, el modelo se debe dividir en sub-modelos cada uno de ellos representando un nivel de abstracción enfocado por un paradigma específico.

Los sistemas complejos normalmente tienen una estructura jerárquica y subsistemas heterogéneos y por lo tanto son difíciles de modelar y simular apropiadamente con un sólo paradigma. Un ejemplo típico de modelamiento de entes heterogéneos es una cadena de suministro que entrega bienes a consumidores de un mercado. El comportamiento del mercado puede ser descrito en términos de la dinámica de sistemas (SD) usando el modelo de Bass, los proveedores, distribuidores y productores pueden ser modelados como agentes (ABS), y las operaciones entre los eslabones de la cadena representados mediante flujogramas de procesos (DES).

El reto en esta línea de investigación involucra diferentes aspectos. El primero es conocer las ventajas y limitaciones de cada uno de los paradigmas. Este trabajo de investigación busca contribuir en este aspecto. El segundo es sincronizar los modelos de simulación que poseen información de diferentes escalas y niveles de abstracción. El mecanismo de alineación debe asegurar que la información de un modelo en una escala determinada debe ser muestreada con una frecuencia determinada antes de ser transferida. Estas muestras pueden ser agregadas o desagregadas dependiendo de si el modelo receptor de la información está a un nivel jerárquico más alto o bajo (Sadsad y McDonnell, 2014).

Entre los temas futuros para trabajar en esta área tenemos el desarrollo de una metodología que permita seleccionar de forma estructurada cual de los paradigmas de simulación puede ser el más adecuado para modelar ciertos problemas. Otro tema de investigación por trabajar es el mejoramiento de los algoritmos de sincronización del 
tiempo en modelos de simulación híbrida (incluyen varios paradigmas de simulación) (Alzraiee et al, 2012). 


\section{REFERENCIAS}

Angerhofer, B.J., y Angelides, M.C. (2000). System dynamics modeling in supply chain management: research view. In Proceedings of the 2000 Winter Simulation Conference, Orlando, FL.

Alzraiee, H., Zayed, T., y Moselhi, O. (2012). Methodology for synchronizing discrete event simulation and system dynamics models. In Proceedings of the 2012 Winter Simulation Conference, Berlin, Germany.

Bagrodia, R., Deelman, E., Docy, S., y Phan, T. (1999). Performance prediction of large parallel applications using parallel simulations. In Proceedings of the ACM SIGPLAN Symposium on Principles and Practice of Parallel Programming, New York, USA.

Bass, F.M. (1969). A new product growth for model consumer durables. Management Science, 15(5), 215-227.

Blanchard, B.S. (1991). Systems Engineering Management. New York: John Wiley \& Sons.

Behdani, B. (2012). Evaluation of paradigms for modeling supply chains as complex sociotechnical systems. In Proceedings of the 2012 Winter Simulation Conference, Berlin, Germany.

Borshchev, A. (2013). The big book of simulation modeling: multimethod modeling with AnyLogic 6.0. AnyLogic North America.

Borshchev, B. \& Filippov, A. (2004). From system dynamics and discrete event to practical agent based modeling: Reasons, techniques, tools. In Proceedings of the 22nd System Dynamics Conference, Oxford, England.

Brailsford, S. (2014a). Discrete-event simulation is alive and kicking!. Journal of Simulation, 8(1), 1-8.

Brailsford, S. (2014b). Theoretical comparison of discrete-event simulation and system dynamics. In S. Brailsford, L. Churilov \& B. Dangerfield (Eds.), Discrete-event simulation and system dynamics for management decision making (pp. 105124). United Kingdom: John Wiley \& Sons.

Brailsford, S.C., Desai, S.M., y Viana, J. (2010). Towards the holy grail: combining system dynamics and discrete-event simulation in healthcare. In Proceedings of the 2010 Winter Simulation Conference, Baltimore, Maryland.

Dangerfield, B. (2014). Systems thinking and system dynamics: A premier. In S. Brailsford, L. Churilov \& B. Dangerfield (Eds.), Discrete-event simulation and system dynamics for management decision making (pp. 26-65). United Kingdom: John Wiley \& Sons. 
Electric Bikes Utah. (2015). Things You Must Know About Eco-Friendly Utah Electric Bikes. Recuperado de http://www.electricbikesutah.com/things-you-must-knowabout-eco-friendly-utah-electric-bikes/

Ford, D.N. (1999). A behavioral approach to feedback loop dominance analysis. System Dynamics Review, 15(1), 3-36.

Forrester, J.W. (1958). Industrial dynamics: a mayor breakthrough for decision makers. Harvard Business Review, 36(4), 37-66.

Forrester, J.W. (1990). Principles of Systems. Portland, OR: Productiviy Press.

Gómez-García, J., y Carmona-Martínez, M. (2003). Modelos de difusión de innovaciones. Aplicación a la agricultura ecológica en España. Anales de Economía Aplicada.

Harrell, C., Ghosh, B.K., y Bowden, R.O. (2004). Simulation using Promodel. New York: McGraw-Hill.

Hayward, J. (2012). Model behavior and the strengths of causal loops: Mathematical insights and a practical method. In Proceedings of the 30th System Dynamics Conference, St Gallen, Switzerland.

Heath, S.K., Brailsford, S.C., Buss, A., y Macal, S.M. (2011). Cross-paradigm simulation mdelling: Challenges and successes. In Proceedings of the 2011 Winter Simulation Conference, Phoenix, Arizona.

Herrera-Madrid, M. (2010). Modelación de la difusión de la tecnología intrasectorial: El caso de las estaciones de servicio de GNV en el valle de Aburrá (Tesis de maestría). Universidad Nacional de Colombia, Medellín, Colombia.

Hitoshi, I. (2013). Agent-based modeling and simulation with Swarm. Boca. Raton: Chapman \& Hall/CRC.

Huang, J. (2012). Analytical and computational methods for analyzing feedback structure in system dynamics models (Doctoral dissertation). National University of Ireland, Galway, Ireland.

Kampmann, C. (2012). Feedback loop gains and system behavior (1996). System Dynamics Review, 28(4), 370-395.

Karnon, J., Stahl, J., Brennan, A., Caro, J.J., Mar, J., y Möller, J. (2012). Modeling using discrete event simulation: A report of the ISPOR-SMDM Modeling Good Research Practices Task Force-4. Value in Health, 15, 821-827.

Kelton, W.D., Sadowski, R.P., y Sturrock, D.T. (2006). Simulation with ARENA. New York: McGraw-Hill.

Kesaraju, V.S., y Ciarallo, F.W. (2012). Integrated simulation combining processdriven and event-driven models. Journal of Simulation, 6(1), 9-20.

Law, A.M. (2014). Simulation modeling and analysis. New York:McGraw-Hill. 
Macal, C.M. (2010). To agent-based simulation from system dynamics. In Proceedings of the 2010 Winter Simulation Conference, Baltimore, Maryland.

Macal, C.M., y North, M.J. (2006). Tutorial on agent-based modeling and simulation part 2: How to model with agents. In Proceedings of the 2006 Winter Simulation Conference, Monterey, California.

Majid, M.A., Aickelin, U., y Siebers, P-O. (2009). Comparing simulation output accuracy of discrete event and agent based models: A quantitative approach. In Proceedings of the Summer Comnputer Simulation Conference (SCSC 2009), Istanbul, Turkey.

Marshall, D.A, Burgos-Liz, L., y IJzerman, M.J. (2015a). Applying dynamic simulation modeling methods in health care delivery research-The SIMULATE checklist: Report of the ISPOR Simulation Modeling Emerging Good Practices Task Force. Value in Health,18,5-16.

Marshall, D.A, Burgos-Liz, L., IJzerman, M.J., Crown, W., Padula, W.V., Wong, P.K.,... Nathaniel, D.O (2015b). Selecting a dynamic simulation modeling method for health care delivery research-Part 2: Report of the ISPOR Dynamic Simulation Modeling Emerging Good Practices Task Force. Value in Health,18,147-160.

Milivojević, N., Grujović, N., Stojanović, B., Divac, D., y Milivojević, V. (2009). Discrete events simulation model applied to large-scale hydro-systems. Journal of the Serbian Society for Computational Mechanics, 3(1), 250-272.

Mojtahedzadeh, M., Andersen, D., y Richarson, G. (2004). Using digest to implement the pathway participation method for detecting influential system structure. System Dynamics Review, 20(1), 1-20.

Morecroft, J. (2007). Strategic modelling and business dynamics: A feedback systems approach. England: John Wiley.

Morecroft, J., y Robinson, S. (2005). Explaining puzzling dynamics: comparing the use of system dynamics and discrete-event simulation. In Proceedings of the 2005 Winter Simulation Conference, Boston, Massachusetts.

Morlán, I. (2010). Modelo de dinámica de sistemas para la implantación de tecnologías de la información en la gestión estratégica universitaria (Tesis doctoral). Universidad del País Vasco, San Sebastián, España.

Parunak, H.V.D., Savit, R., y Riolo, R. (1998). Agent-based modeling vs equationbased modeling: A case study and users' guide, In Sichman, J., S., Conte, R. \& Gilbert, N. (Eds.) Multi-Agent Systems and Agent-Based Simulation, Lecture Notes in Artificial Intelligence (pp. 10-25). London, United Kingdom: SpringerVerlag.

Pegden, D. (2010). Advanced tutorial: overview of simulation world views. In Proceedings of the 2010 Winter Simulation Conference, Baltimore, Maryland. 
Phaff, H.W.G., Slinger, J.H., Güneralp, B., y van Daalen, C.E. (2006).Investigating model behavioural analysis: A critical examination of two methods. In Proceedings of the 24th System Dynamics Conference, Nijmegen, The Netherlands.

Pidd, M. (2004). Computer simulacion in management science. New York: John Wiley $\&$ Sons.

Pritsker, A.A.B., y O'Really, J.J.O. (1999). Simulation with Visual Slam and Awesim. New York: John Wiley \& Sons.

Rabelo, L., Helal, M., Jones, A., Min, J., Son, Y.-J., y Deshmukh, A. (2003). A hybrid approach to manufacturing enterprise simulation. In Proceedings of the 2003 Winter Simulation Conference, New Orleans, LA.

Railsback, S.F., y Grimm, V. (2011). Agent-based and individual-based modeling: a practical introduction. Princeton: Princeton University Press.

Ramírez, N.E. (2014). Application of system dynamics to model rework in construction projects (Tesis de maestría). Universidad Politécnica de Cataluña, Barcelona, España.

Richardson, G. (1995). Loop polarity, loop dominance, and the concept of dominant polarity. System Dynamics Review, 11(1), 67-88.

Robinson, S. (2004). Simulation: The practice of model development and use. John Wiley \& Sons, Chichester, UK.

Robinson, S. (2014). Discrete-event simulation: A premier. In: S. Brailsford, L. Churilov \& B. Dangerfield, ed. Discrete-event simulation and system dynamics for management decision making. United Kingdom: John Wiley \& Sons, 10-25.

Rockwell Software Inc. (2010). Arena user's guide, USA, WI, Milwaukee.

Sadsad, R., y McDonnell, G. (2014). Multiscale modelling for public health management: A practical guide. In S. Brailsford, L. Churilov \& B. Dangerfield (Eds.), Discrete-event simulation and system dynamics for management decision making (pp. 281-294). United Kingdom: John Wiley \& Sons.

Salamon, T. (2011). Design of agent-based models. Czech Republic: Tomas Bruckner Publishing.

Serova, E. (2013). The role of agent based modelling in the design of management decision processes. The Electronic Journal Information Systems Evaluation, 16(1), 71-80.

Schieritz, N., y Milling, P. (2003). Modeling the forest or modeling the threes - a comparison of system dynamics and agent-based simulation. In Proceedings of the 21st System Dynamics Conference, New York, USA. 
Siebers, P.O., Macal, C.M., Garnet, J., Buxton, D., y Pidd, M. (2010). Discrete-event simulation is dead, long live agent-based simulation!. Journal of Simulation, 4(3), 204-210.

Skoogh, A., y Johansson, B. (2008). A methodology for input data management in discrete event simulation projects. In Proceedings of the 2008 Winter Simulation Conference, Miami, Florida.

Sterman, J. (2000). Business dynamics: Systems thinking and modeling for a complex world. Boston, Massachusetts: McGraw-Hill.

Sumari, S., Ibrahim, R., Zacaria, N.H., y Ab Hamid, A.H. (2013). Comparing three simulation model using taxonomy: System dynamic simulation, discrete event simulation and agent based simulation. International Journal of Management Excellence, 1(3), 54-59.

Sweetser, A. (1999). A Comparison of system dynamics (SD) and discrete event simulation (DES). In Proceedings of the 1999 Winter Simulation Conference and the 5th Australian \& New Zealand Systems Conference, Wellington, New Zealan.

Tacko, A.A., y Robinson, S. (2009). Comparing model development in discrete event simulation and system dynamics. In Proceedings of the 2009 Winter Simulation Conference, Austin, Texas.

Taylor, R. (1990). Interpretation of the correlation coefficient: A basic review. Journal of Diagnostic Medical Sonography, 6, 35-39.

Vangheluwe, H. (2001). Discrete event modelling and simulation. Recuperado de http://www.cs.mcgill.ca/ hv/classes/MS/discreteEvent.pdf

Ventana Systems, Inc. (2013). Vensim reference manual.

Wakeland, W.W., Gallager, E.J., Makovsky, L.M., y Aktipis, C.A. (2004). A comparison of system dynamics and agent-based simulation applied to the study of cellular receptor dynamics. In Proceedings of the the 37th Annual Hawaii International Conference on System Sciences, Big Island, Hawaii.

Wilensky, U. (1999). NetLogo. http://ccl.northwestern.edu/netlogo/. Center for Connected Learning and Computer-Based Modeling, Northwestern University, Evanston, IL.

Yu, T-T. (2008). The development of a hybrid simulation modelling approach based on agents and discrete-event modelling (Doctoral dissertation). University of Southampton, England. 
ANEXOS 


\section{ANEXO 1: Código en ARENA del modelo de negocio de ESR}

NOTA: Como ARENA no tiene definida la unidad de tiempo "quincena" se usó la etiqueta de unidad de tiempo "Minutes". Sin embargo, al ser sólo una etiqueta no altera los datos del modelo que fueron ingresados todos en función de quincenas.

\section{$\underline{\text { Archivo .exp }}$}

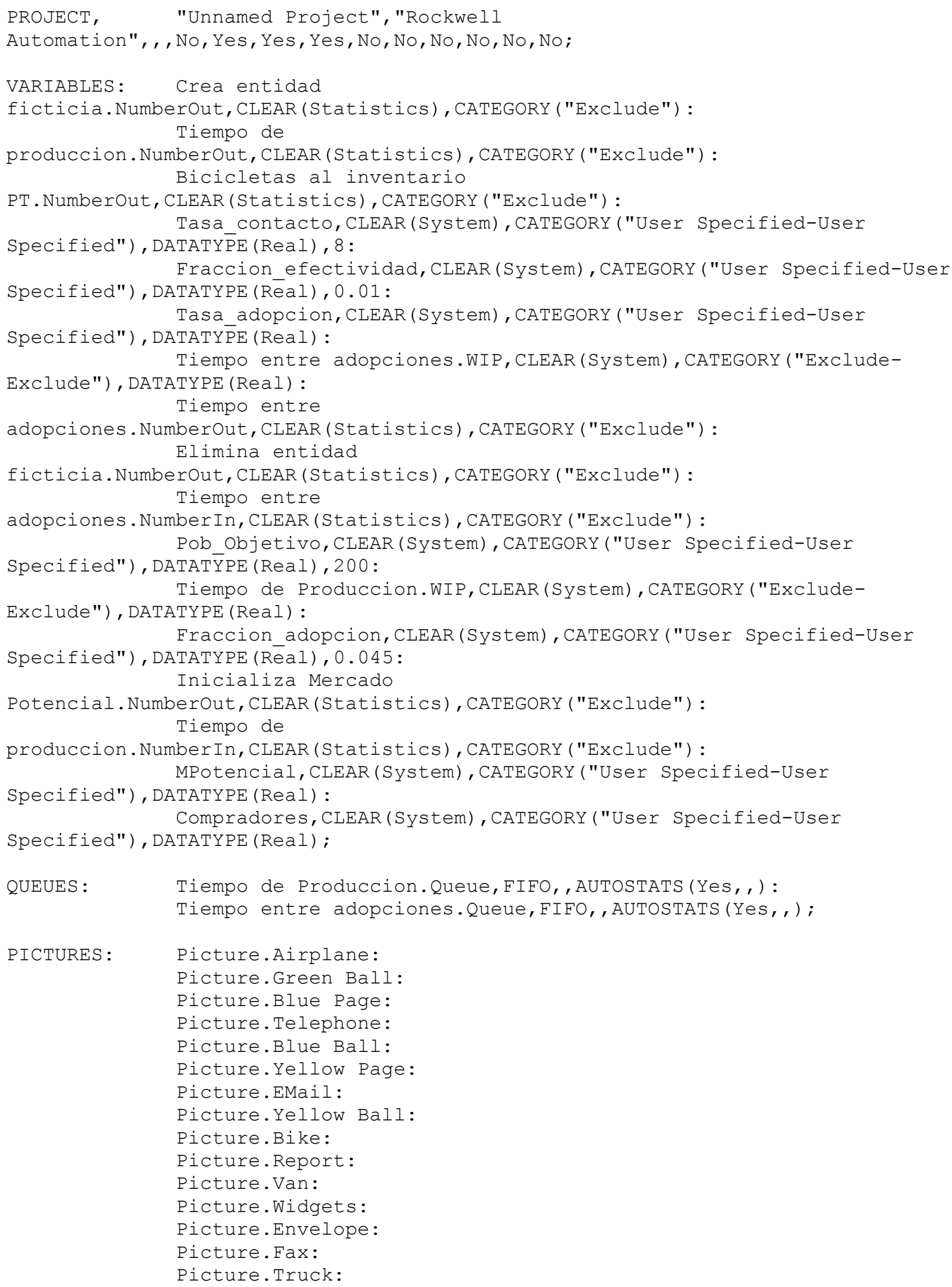




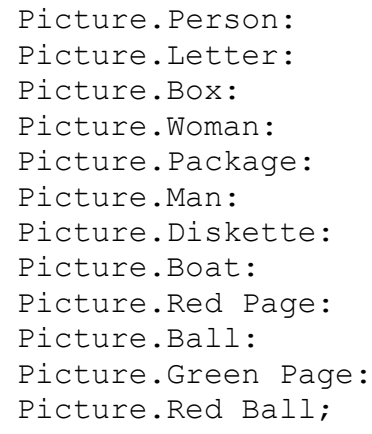

RESOURCES :

Linea produccion, Capacity (2), , $\operatorname{COST}(0.0,0.0,0.0)$, CATEGORY (Resources), ,AUTOSTAT S $\left(\right.$ Yes, $\left._{1},\right)$ :

Recurso_adopcion, Capacity (1), , $\operatorname{COST}(0.0,0.0,0.0)$, CATEGORY (Resources), ,AUTOSTAT $\mathrm{S}($ Yes,,$\overline{)}$;

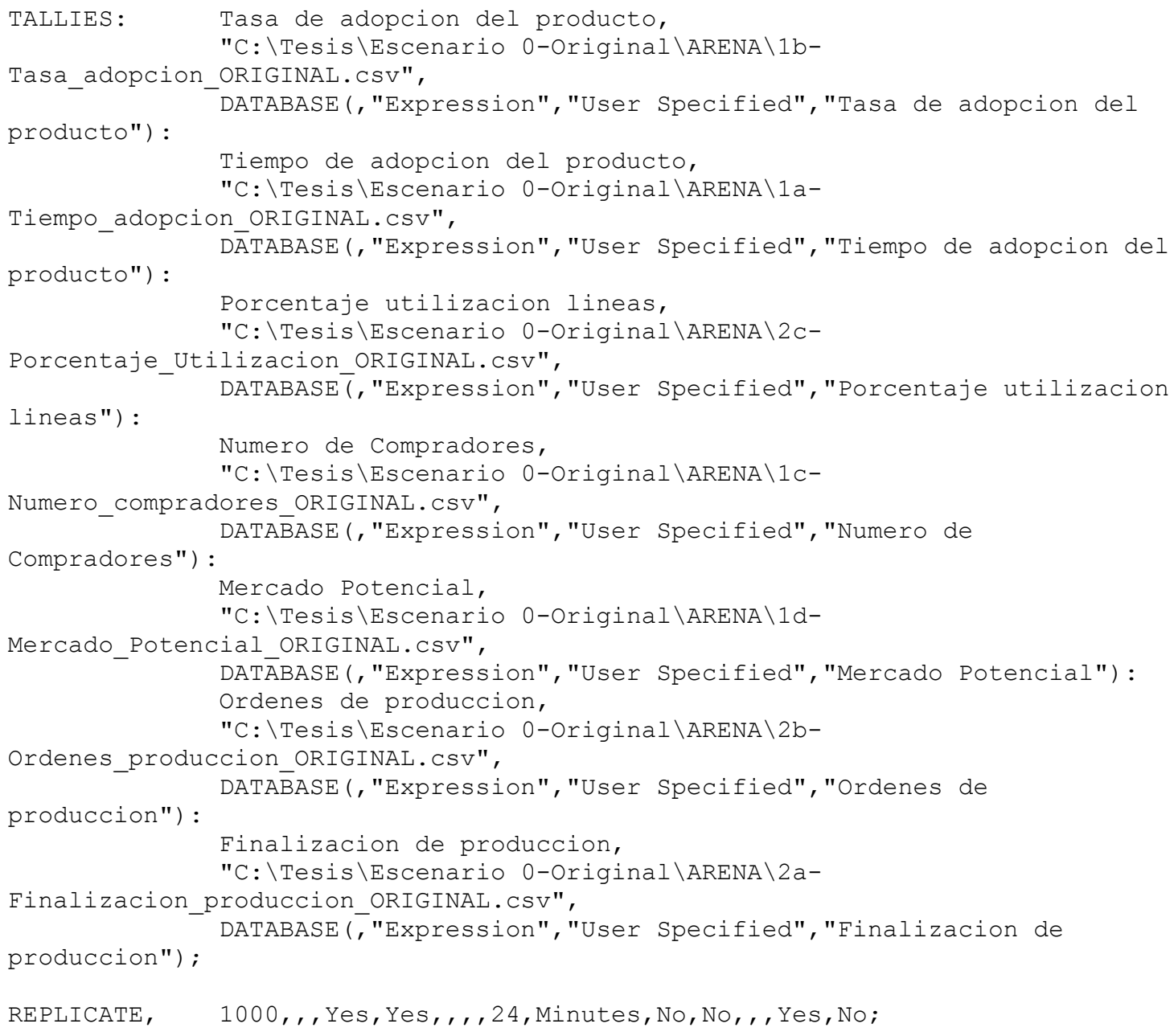


; Model statements for module: BasicProcess.Assign 1 (Actualizacion variables adopcion)

$1 \$$

ASSIGN :

Compradores $=$ Compradores +1 :

MPotencial=Pob_Objetivo-Compradores:

Tasa_adopcion=Adopcion_boca_a_boca+Adopcion_publicidad:NEXT (2\$);

; Model statements for module: BasicProcess.Record 1 (Graba Tiempo de Adopcion)

$2 \$$

TALLY:

Tiempo de adopcion del producto, tnow, 1:NEXT(3\$);

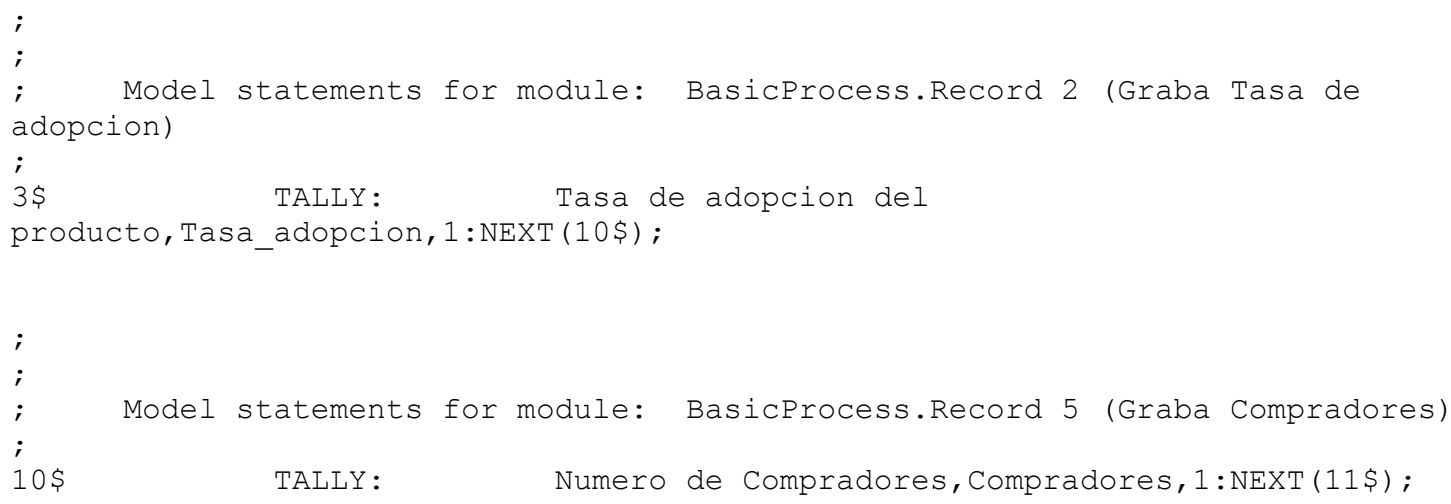


; Model statements for module: BasicProcess.Record 3 (Graba Finalizacion de Produccion)

;

$8 \$$

TALLY :

Finalizacion de produccion, tnow, $1: \operatorname{NEXT}(9 \$)$

; Model statements for module: BasicProcess.Record 4 (Graba Ordenes de Produccion)

9\$ TALLY: Ordenes de produccion, Tiempo de

produccion.WIP, 1 : NEXT (12\$);

; Model statements for module: BasicProcess.Record 7 (Graba Utilizacion de las Lineas)

$12 \$$ TALLY: Porcentaje utilizacion

lineas, ResUtil (Linea produccion) *100,1:NEXT (0\$);

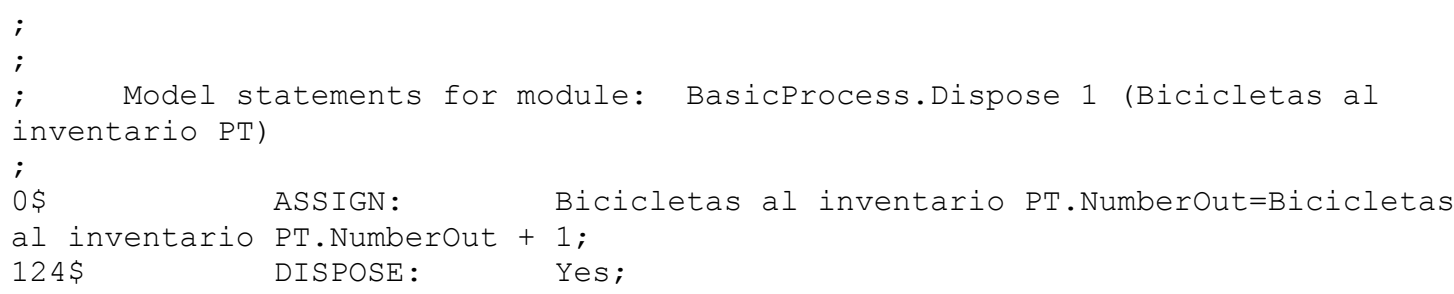




\section{ANEXO 2: Código en VENSIM del modelo de negocio de ESR}

NOTA: Como VENSIM no tiene definida la unidad de tiempo "quincena" se usó la etiqueta de unidad de tiempo "month". Sin embargo, al ser sólo una etiqueta no altera los datos del modelo que fueron ingresados todos en función de quincenas.

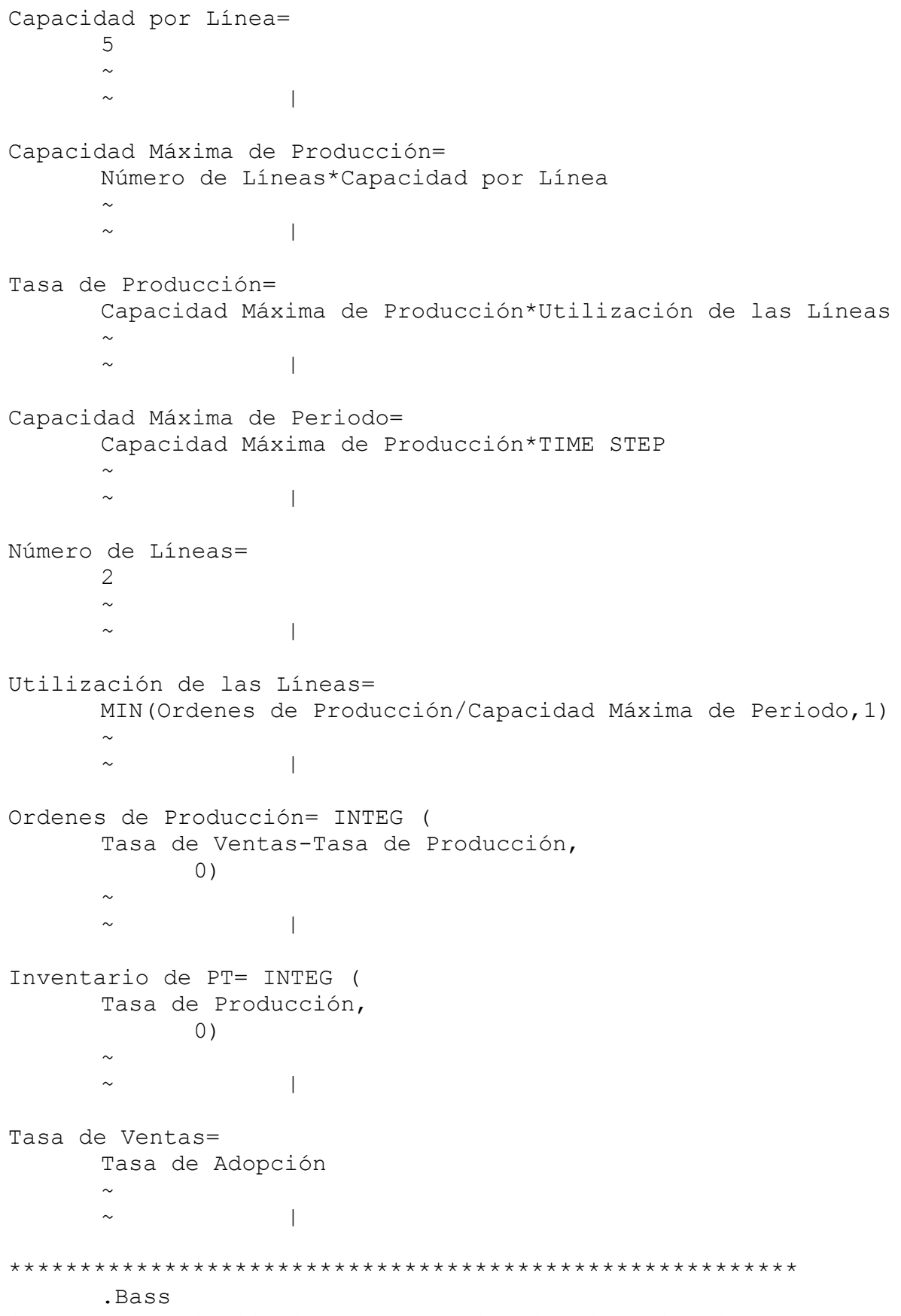




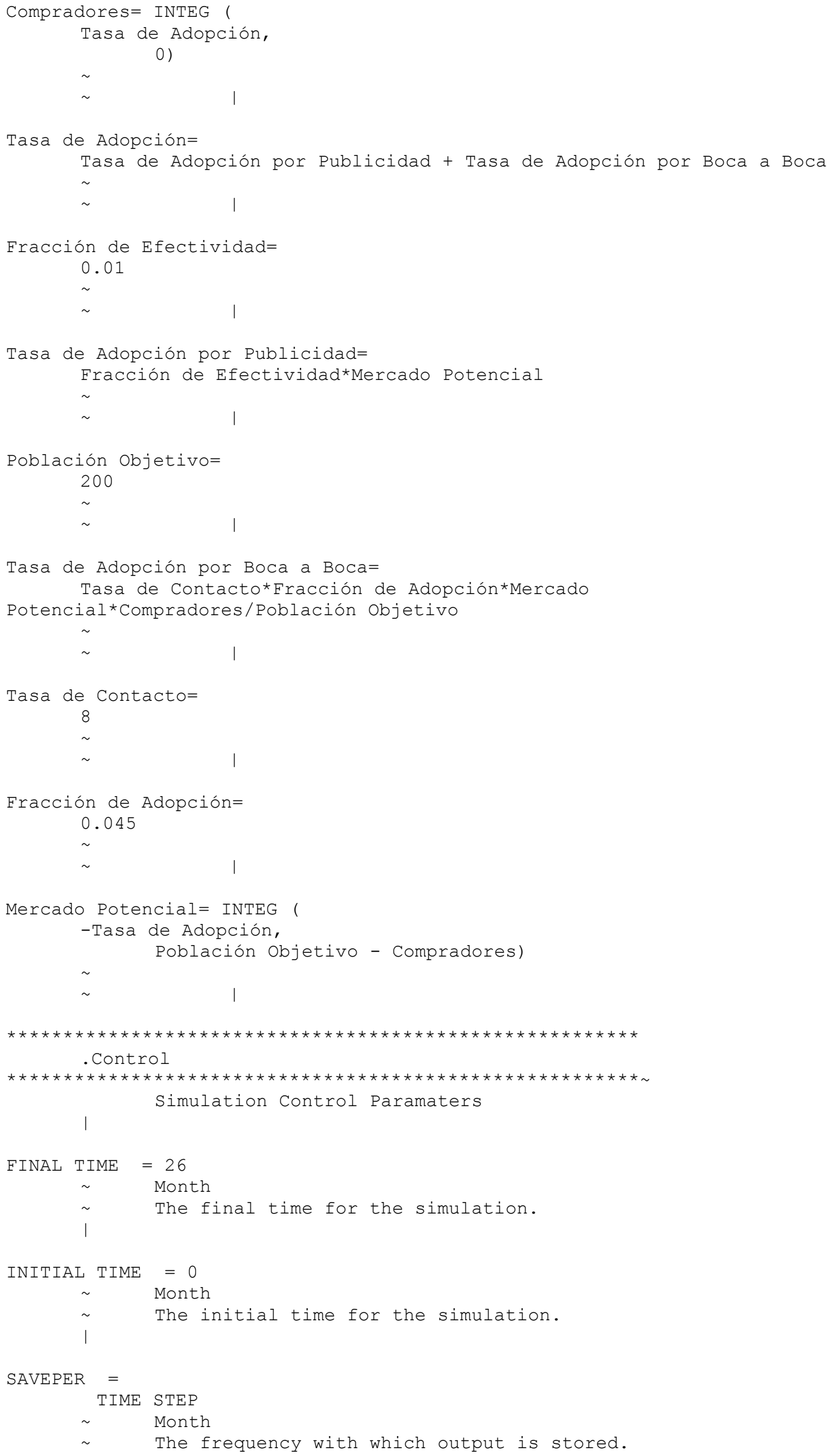


TIME STEP $=0.25$

Month 


\section{ANEXO 3: Código en NETLOGO del modelo de negocio de ESR}

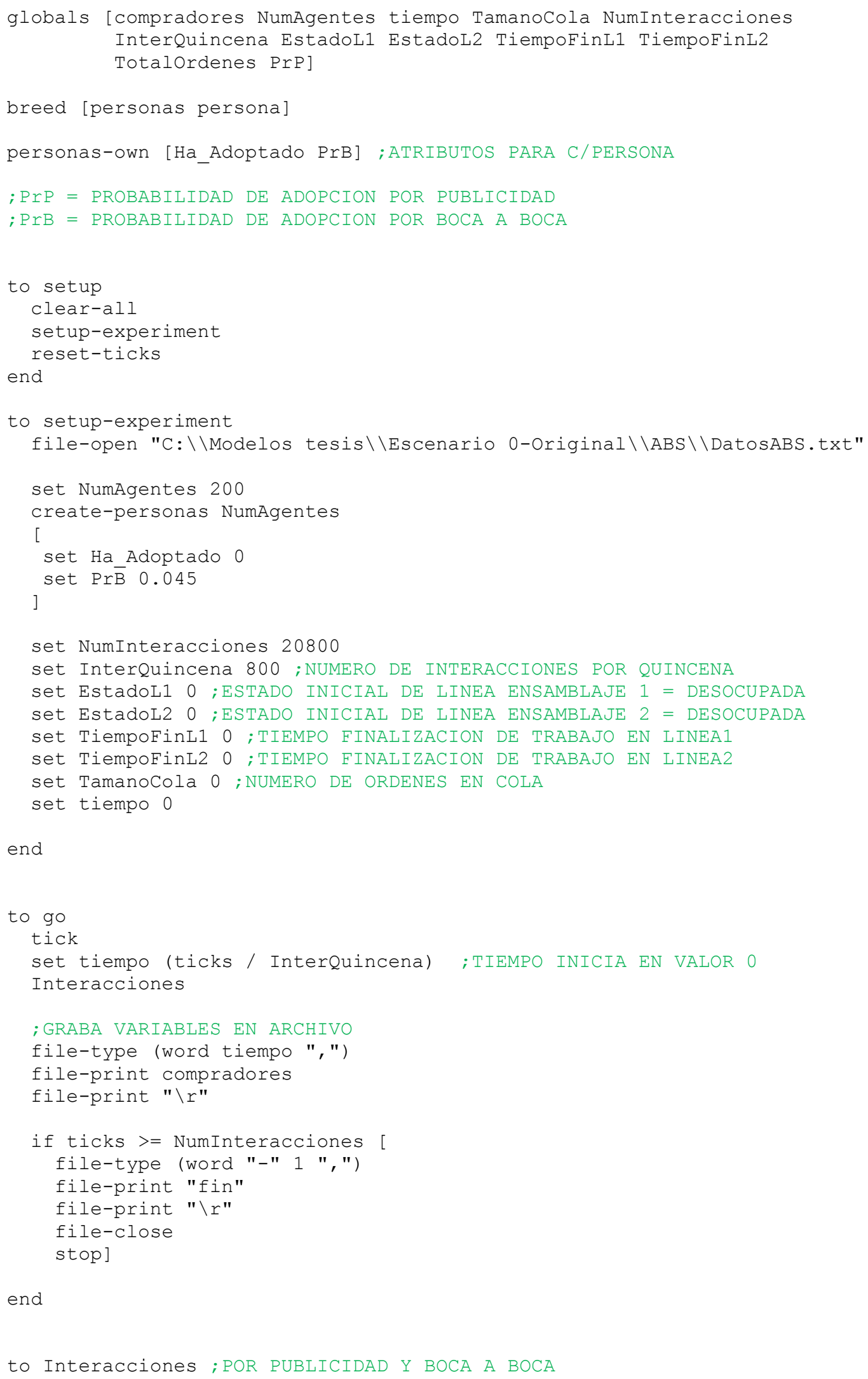




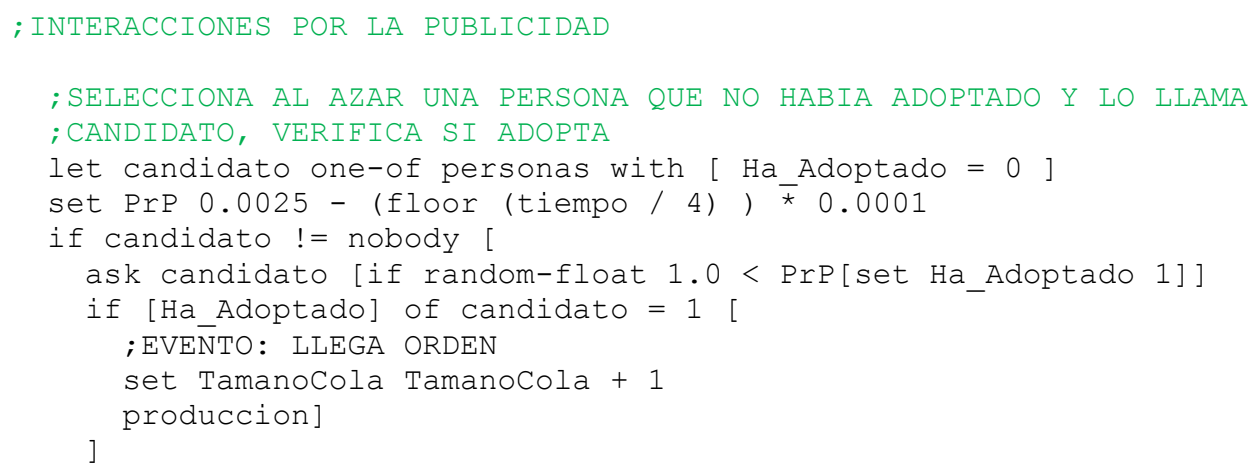




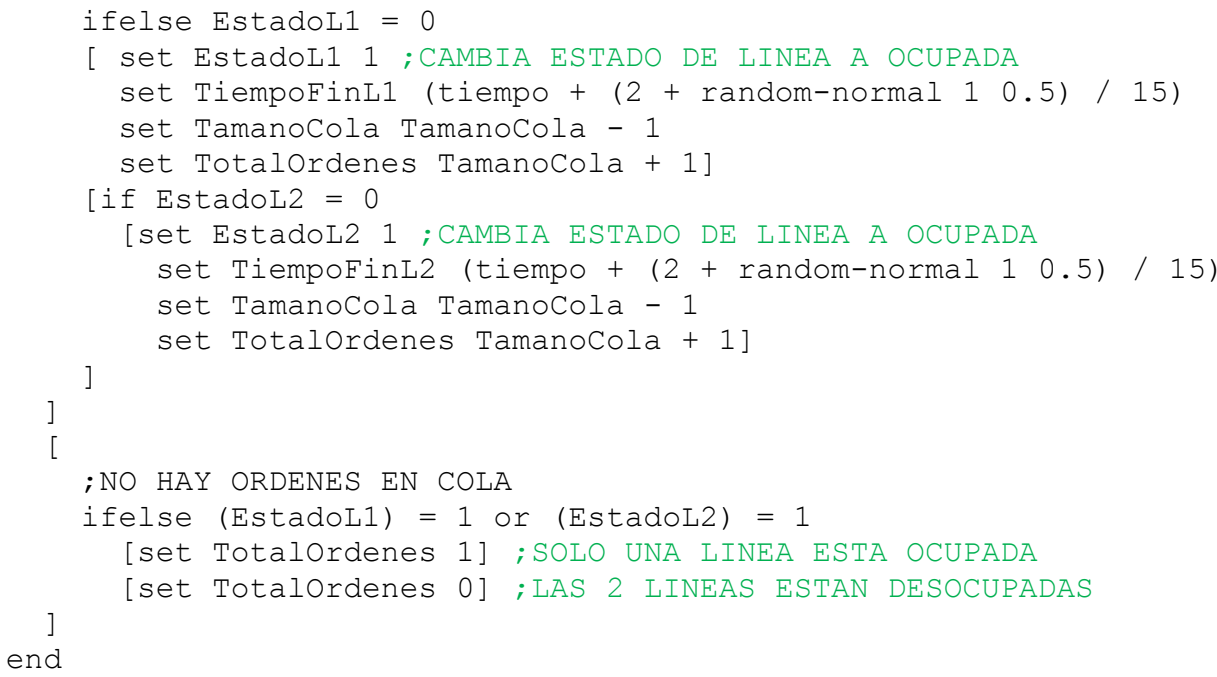

